\title{
Investigations of azulene derivatives as self- indicating chromophores for applications in protecting group strategies and solid-phase purification
}

\author{
By
}

Thomas Bevan

\section{Victoria \\ UNIVERSITY OF WELLINGTON \\ Te Whare Wänanga \\ o te Upoko o te Ika a Măui \\ 푹둥용}

\begin{abstract}
A thesis
submitted to Victoria University of Wellington

in fulfilment of the requirements for the degree of

Master of Science in Chemistry
\end{abstract}

Victoria University of Wellington

2010 


\section{Abstract}

Azulene (1) is a hydrocarbon that exhibits an intense blue colour. Derivatives of azulene exhibit different colours depending on the position and electronic properties of the substituents. The chemical and chromophoric properties of azulene and guaiazulene (2) derivatives are investigated for the potential use in applications such as chromophoric protecting groups and self-indicating scavenger resins.

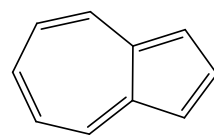

1

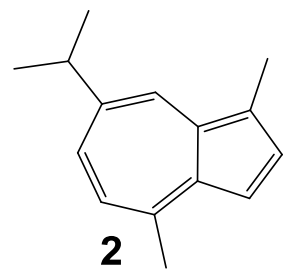

Investigations were carried out on the scope of known reactions on the 3-position of guaiazulene for this purpose. These included Vilsmeier-Haack formylation and acylation with oxalyl halides.

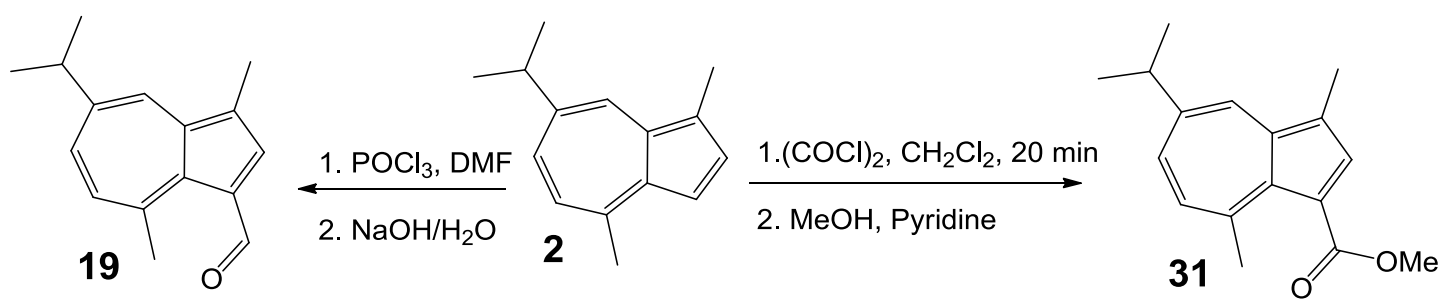

A method for the reductive amination of $\mathbf{1 9}$ was developed and considered as a protecting group strategy. A novel method of synthesising esters such as $\mathbf{3 1}$ using oxalyl chloride was developed, and this chemistry was investigated in Friedel-Crafts acylation of other arenes.

The deprotonation of the 4-methyl position of guaiazulene was investigated for its scope and limitations. Several electrophiles such as alkyl halides, carbonyl-containing compounds and silyl chlorides were added to the guaiazulenide anion $\mathbf{3 5}$ to investigate reactivity and identify potential protecting group syntheses. Deprotonation of derivatives such as $\mathbf{3 1}$ with LDA were also investigated.

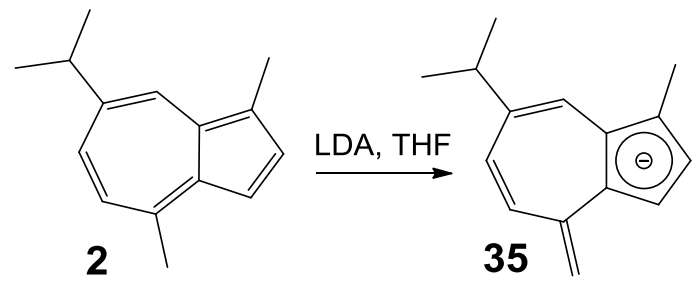


A potential protecting group system was identified for carboxylic acids which had an FMOC-analogous deprotection mechanism. The carboxylic ester was successfully removed using the mild base piperidine, thus demonstrating the potential usefulness in synthesis that these azulenes may have as protecting groups.
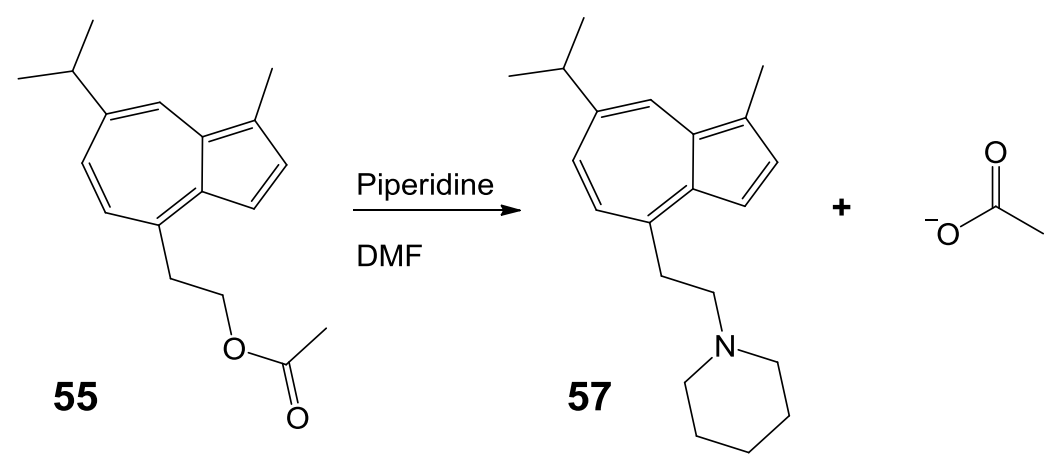

A base-labile protecting group for diols $(\mathbf{6 3})$ with a similar deprotection step was proposed, and several attempts were made to synthesise it without success.

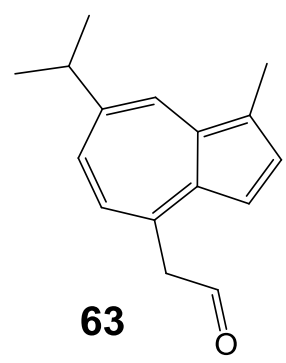

The guaiazulenide anion $\mathbf{3 5}$ was also added to Merrifield resin, to create a guaiazulene-functionalised polystyrene-divinylbenzene polymer resin. Further reactions were performed on this resin which demonstrated its possible use as a scavenger resin. Difficulties were encountered in characterisation, as aside from a visual colour change and a change in mass of the resins, little could be done spectroscopically to characterise these resins.

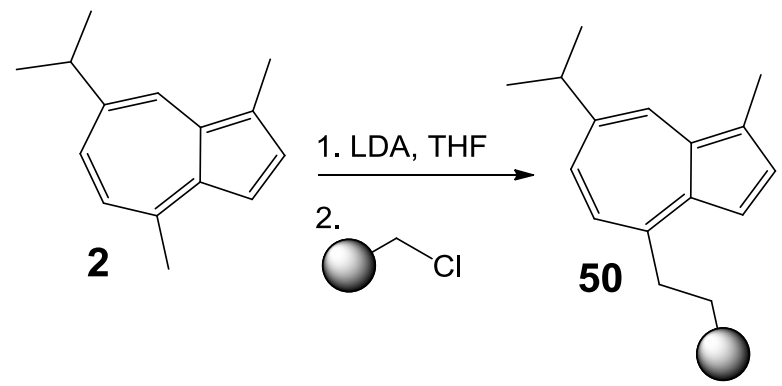




\section{Dedication}

To me dad. 


\section{Acknowledgements}

"Do not go gentle into the valley of death, Rage, rage against the dying of the six hundred"

-Dylan Lord Thomasson

Firstly, thanks go to my primary supervisor A/Prof Peter Northcote, who provided many ideas and invaluable guidance, despite the difficulties associated with a project that was sometimes beyond his (or anyone else's for that matter) expertise. Also, thanks go to Dr. Brendan Burkett for being an excellent supervisor during the beginning of this project.

Of the other academics, special thanks go to Dr. Joanne Harvey for taking me into her research group as one of her own after the departure of Dr. Burkett, and to A/Prof Paul Teesdale-Spittle for numerous insightful comments and proofreading. Thanks also go to Dr. Matthias Lein for help with computational chemistry, and to Em/Prof Brian Halton for many useful pieces of advice, use of the Spartan software, and numerous intriguing tales of chemistry as performed in 'the dark ages'.

Of my labmates, special thanks go to the long suffering senior PhD student Russell 'Russocles' Hewitt, who provided many helpful words of wisdom, helped tremendously with proofreading and was in general a great friend. Thanks also go to the other labmates Mark, Hemi, Sam, Febly, Johnny and the two Peters; Curly and Stumpy.

Thanks go to many of the other post-grads. I'd like to thank Sean O'Connor, Ishira Samarasinghe, Helen Woolner, Jacqui Barber, Matthias Herzog, Jason Johnstone and the fourth years Joe Gallaher, Bryan Northover and Martin Heeley for their support and camaraderie, and to the $3^{\text {rd }}$ years of 2010 for their occasional comic relief.

Also thanks go to the general SCPS staff, including the ever cheery and helpful Lisa and Sally, and to John Ryan, Grant, Alan, Scotty, Darren and Dan for various things.

Finally, many thanks to Mum, Dad, my sister and my grandparents for all the support and understanding they've given me, both throughout this project and in general. 


\section{Table of Contents}

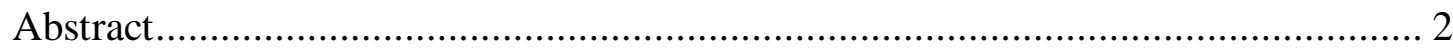

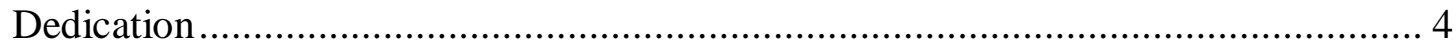

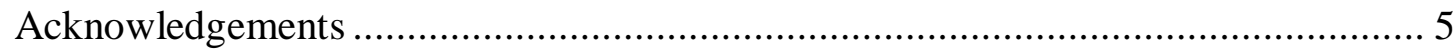

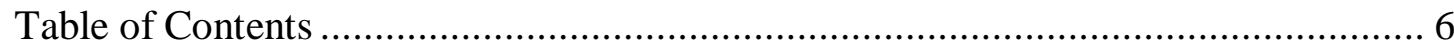

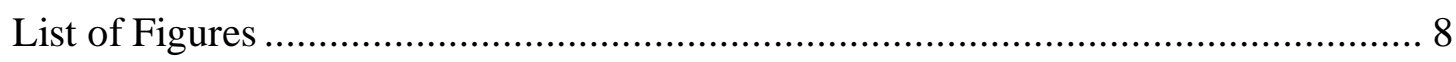

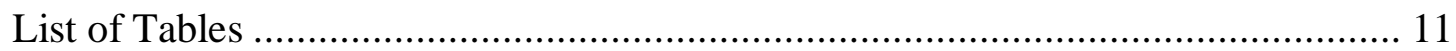

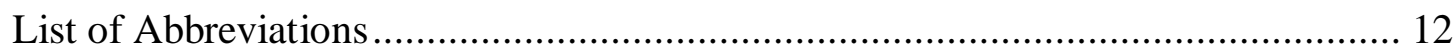

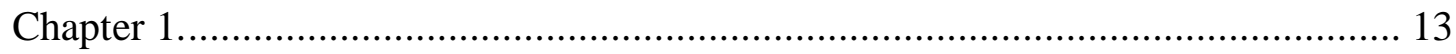

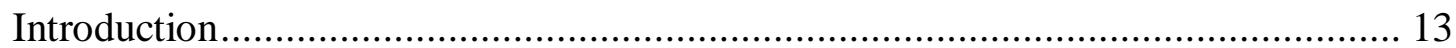

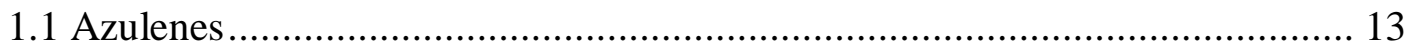

1.1.1 Resonance structure of azulene ……........................................... 14

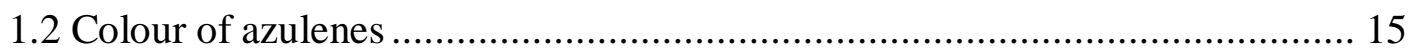

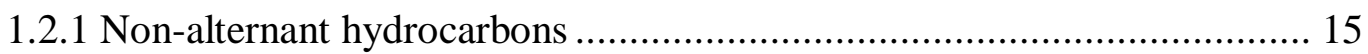

1.2.2 Origin of the small HOMO-LUMO gap ........................................... 17

1.2.3 Colour in azulene derivatives ......................................................... 18

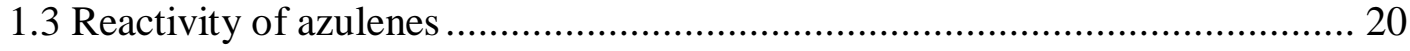

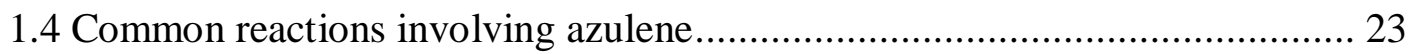

1.4.1 Reactions at the 1 and 3 positions ................................................. 23

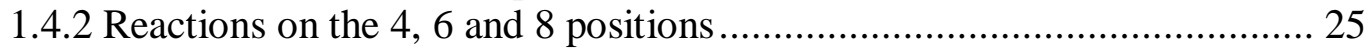

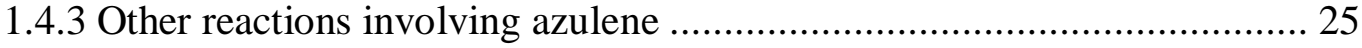

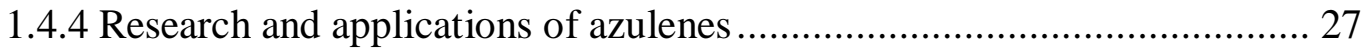

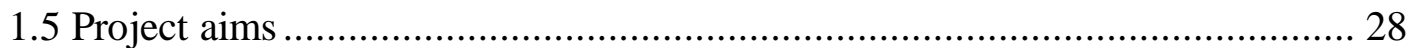

1.6 Azulene derivatives as coloured tags and protecting groups ....................... 28

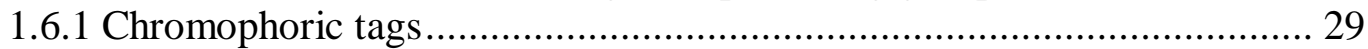

1.6.2 General strategy of protecting group research..................................... 30

1.6.3 Previous examples of azulene-based protecting groups ......................... 31

1.7 Azulene functionalised polystyrene-divinylbenzene resins ........................ 33

1.7.1 Polystyrene-divinylbenzene (PS-DVB) resins .................................... 34

1.7.2 Use of PS-DVB resins in peptide synthesis and combinatorial chemistry 35

1.7.3 Use of PS-DVB as scavenger resins ................................................ 35

1.7.4 Motivation for the functionalisation of PS-DVB resins with azulene

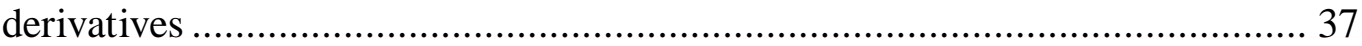

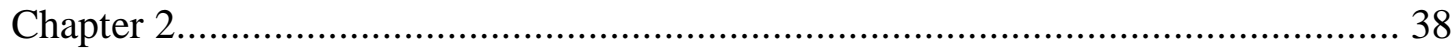

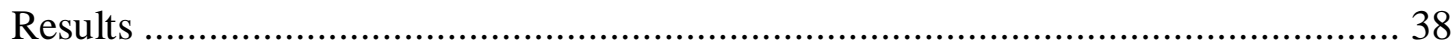

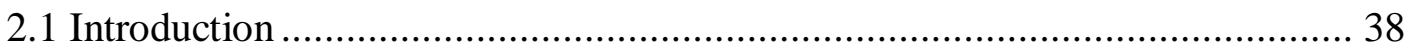


2.2 Reactions on the 3-position of guaiazulene .................................................. 38

2.2.1 Vilsmeier-Haack formylation and related reactions .............................. 39

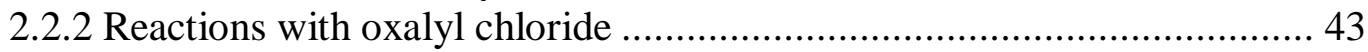

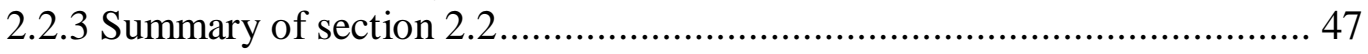

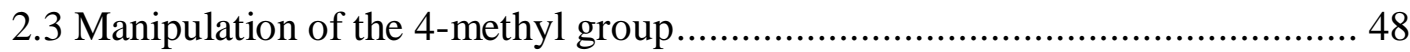

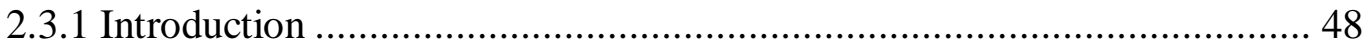

2.3.2 Deprotonation of guaiazulene............................................................ 48

2.3.3 Deprotonation of guaiazulene derivatives ........................................ 51

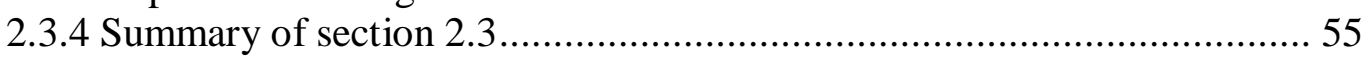

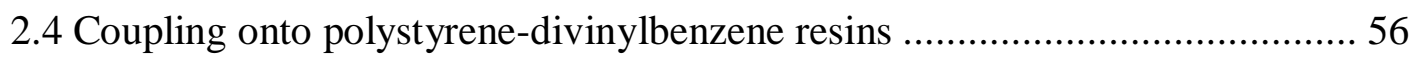

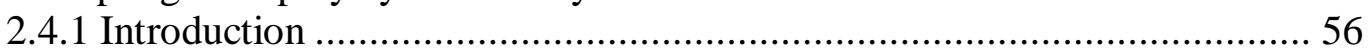

2.4.2 Synthesis of a guaiazulene-functionalised PS-DVB resin ..................... 56

2.4.3 Characterisation of the guaiazulene resin................................................5 58

2.4.4 Investigation of on-resin reactions and use as scavenger resins ............... 59

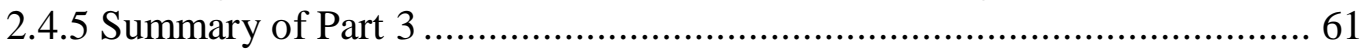

2.5 Towards development of a guaiazulene-based protecting group system ........ 62

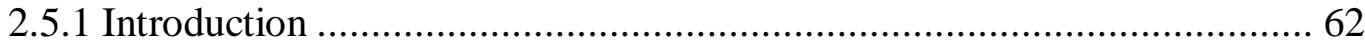

2.5.2 A protecting group for primary amines based on reductive amination and

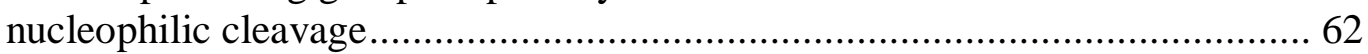

2.5.3 A protecting group for cis-diols based on 3-formyl guaiazulene ............. 62

2.5.4 An FMOC-analogous protecting group ............................................ 63

2.5.5 Towards synthesis of a base-labile diol protecting group ...................... 67

2.5.6 Towards the synthesis of an azulenic silyl protecting group................... 73

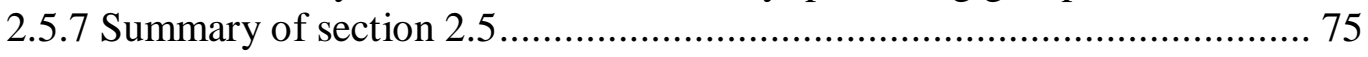

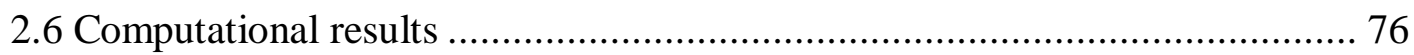

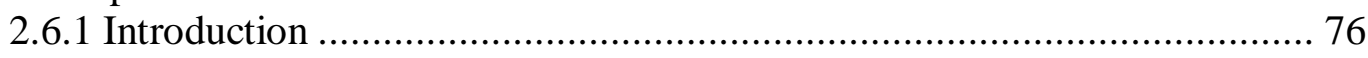

2.6.2 Calculation of the visible absorption spectra of azulene derivatives ........ 76

2.5.3 Calculation of the relative energies of the keto- and enol- forms of the

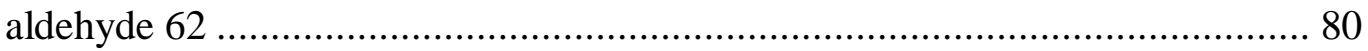

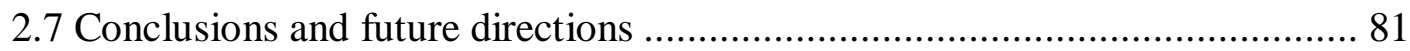

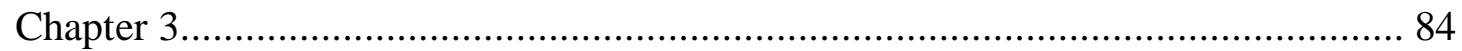

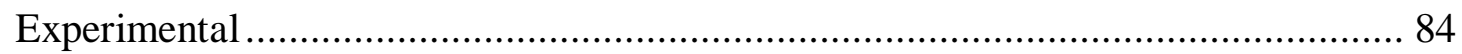

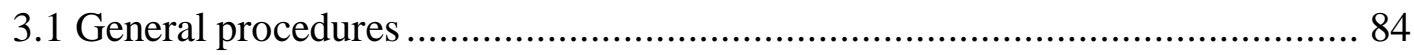

3.1.1 General procedure for LDA deprotonation reactions ......................... 84

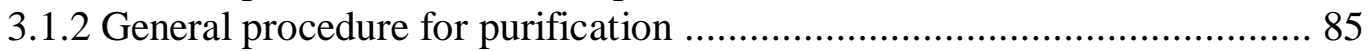

3.1.3 Identification of compounds through the use of TLC........................... 86

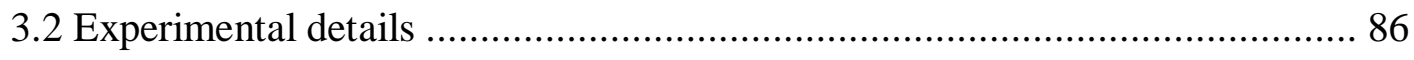

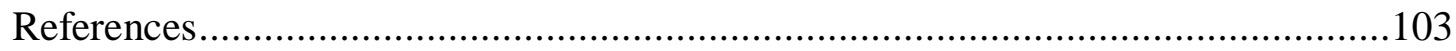




\section{List of Figures}

1.1 The chemical structures of azulene $\mathbf{1}$ and guaiazulene $\mathbf{2} 13$

1.2 Resonance and mesomeric forms of the aromatic ions cyclopentadienide, top and tropylium, bottom 14

$\begin{array}{lll}1.3 & \text { Mesomeric forms of azulene } & 14\end{array}$

$\begin{array}{lll}1.4 & \mathrm{UV}-\mathrm{V} \text { is spectrum of azulene } & 15\end{array}$

1.5 Examples of alternant and non-alternant hydrocarbons 16

1.6 LUMO+4 for naphthalene, left and azulene, right 16

1.7 The HOMO and LUMO of naphthalene, left, and azulene, right 17

$\begin{array}{ll}1.8 & \text { Numbering system of the azulene moiety } \\ & 18\end{array}$

1.9 The effect of EDGs and EWGs at the 1- position on the electronic
structure of azulene

1.10 The effect of EDGs and EWGs at an even position on the electronic
structure of azulene

1.11 Aromatically-stabilised and non aromatically-stabilised charge separated resonance forms of azulene 21

1.12 Resonance structures of the 1-addition intermediate, above, and of the 2-addition intermediate, below 22

1.13 Charge-separated resonance forms of azulene, illustrating the accessibility of the stabilising cyclopentadienide form 22

$\begin{array}{ll}\text { 1.14 The frontier molecular orbitals of azulene } & 23\end{array}$

1.15 Reaction scheme of the Vilsmeier-Haack reaction on azulene 24

$\begin{array}{ll}\mathbf{1 . 1 6} \text { Acylation of azulene } & 24\end{array}$

$\begin{array}{ll}1.17 & \text { Nucleophilic substitution of azulene } \\ 1.18 & 25\end{array}$

1.18 Coupling of 4-methyl azulene with bromoacetic acid 25

1.19 4-step synthesis of azulene from cyclopentadiene, dimethylformamide $\begin{array}{ll}\text { diethylacetal and butadiene sulfone } & 26\end{array}$

1.20 Synthesis of 4,6,8-trimethyl azulene 2-methyl ester 26

1.21 Synthesis of tris(azulenyl)methane from azulene and 1-formyl azulene, as reported by $\mathrm{S}$. Ito

1.22 Protection of an alcohol using the carboxylic acid $\mathbf{1 4} 27$

$\mathbf{1 . 2 3}$ Fluorescein $\mathbf{9}$ and the dansyl group $\mathbf{1 0}$ are examples of groups commonly used as fluorescent tags

1.24 The orange-red ( $p$-phenylazophenyl)isopropyloxycarbonyl (AZOC) protecting group

1.25 A generalised structure of an electronically connected (left) and an electronically isolated (right) protecting group

1.26 Reaction of azulene with oxalyl chloride followed by addition of the alcohol generates the ketoester-protected compound $\mathbf{1 2}$

1.27 Addition of $o$-diaminobenzene to $\mathbf{1 2}$ in refluxing acetic acid cleaves the alcohol in high yield

1.28 Preparation of the protecting group $\mathbf{1 4}$ followed by activation with carbonyldiimidazole

1.29 Coupling of the guaiazulene derivative $\mathbf{1 5}$ to the mannoside $\mathbf{1 6}$ gave the protected mannoside $\mathbf{1 7}$ in decent yield

1.30 General cleavage conditions for the guaiazulene-based protecting group 33

1.31 Illustration of the interior polymeric structure of non-swelled and 34 swelled resins

1.32 Formation of PS-DVB resin 
1.33 Examples of scavenger resins with different functions 36

1.34 Example of synthesis performed using only scavenger resins and filtration as purification

2.1 Generation of Vilsmeier reagent from $\mathrm{POCl}_{3}$ and DMF

2.2 Reaction of $\mathbf{2}$ with Vilsmeier reagent followed by hydrolysis generates the aldehyde 19

2.3 Reductive amination of the imine $\mathbf{2 0}$ to produce the amine $\mathbf{2 2}$

2.4 General procedure for the synthesis of azulene-functionalised secondary amines

2.5 Proposed nucleophilic attack and deprotection of the amine 7. $\mathrm{S}_{\mathrm{N}} 2$ mechanism, top and $\mathrm{S}_{\mathrm{N}} 1$ mechanism, bottom

2.6 Use of one equivalent of cyclohexylamine gave rise to a 1:1 mixture of the products $\mathbf{2 4}$ and $\mathbf{2 2}$

2.7 Proposed mechanism for the degradation of amine $\mathbf{2 2}$ into the bis(guaiazulenyl)methane $\mathbf{2 5}$.

2.8 Reaction reported by Lash et al. in which ipso-substitution of $\mathbf{2 6}$ and loss of formaldehyde results in the formation of $\mathbf{2 7}$

2.9 Reduction of the aldehyde $\mathbf{1 9}$ resulted in the formation of $\mathbf{2 9}$

2.10 Formation of an aryl ester from an arene using oxalyl chloride and an alcohol

2.11 Formation of the ketoester $\mathbf{3 0}$ from $\mathbf{2}$ using oxalyl chloride

2.12 Formation of the ester $\mathbf{3 1}$ from $\mathbf{2}$ using oxalyl bromide

2.13 Proposed radical-based conversion mechanism from keto-acid chloride to acid chloride

2.14 Synthesis of the amide 32 from 2

2.15 General form of the attempted syntheses of ketone $\mathbf{3 3}$ from 2

2.16 The guaiazulene ethyl ester $\mathbf{3 4} 46$

2.17 Postulated identity of the crimson-red compound 46

2.18 Deprotonation of guaiazulene at the 4-methyl position affords the guaiazulenide anion $\mathbf{3 5}$

2.19 Nucleophilic attack by 35 on formaldehyde affords the alcohol 36

2.20 Reaction of $\mathbf{3 5}$ with benzyl chloride produces $\mathbf{3 7}$

2.21 The guaiazulenide anion $\mathbf{3 5}$ reacts with dibromoethane to produce $\mathbf{3 8} 50$

2.22 The bromide $\mathbf{3 8}$ was converted to the thiol $\mathbf{3 9}$

$\mathbf{2 . 2 3}$ The derivatives 40, 41, $\mathbf{4 2}$ were synthesised from $\mathbf{3 7} 51$

2.24 Postulated degradation products of the guaiazulenide ester $\mathbf{4 3}$

2.25 The lactone $\mathbf{4 4}$ was the only identifiable product from the deprotonation 53 and subsequent treatment of $\mathbf{3 1}$ with paraformaldehyde

2.26 The formation of $\mathbf{4 6}$ was attributed to benzaldehyde impurity in the benzyl chloride reagent

2.27 The ester $\mathbf{4 7}$ was synthesised from guaiazulene using oxalyl chloride and tert-butanol

2.28 Deprotonation of $\mathbf{4 7}$ to form the anion $\mathbf{4 8}$, which was reacted with several different electrophiles

2.29 Radical initialised polymerisation to form functionalised PS-DVB, with Az representing an arbitrarily attached azulene derivative

2.30 Benzyl chloride, left, and three depictions of Merrifield resin, right $\quad 57$

$\mathbf{2 . 3 1}$ Formation of resin $\mathbf{5 0}$

2.32 Photograph of the guaiazulene resin $\mathbf{5 0}$

2.33 Attempted oxalyl chloride acylation on the resin $\mathbf{5 0} 60$

$\mathbf{2 . 3 4}$ Attempted Vilsmeier-Haack formylation of $\mathbf{5 0}$ 
$\begin{array}{lll}\text { 2.35 First investigated protecting group candidates } & 62\end{array}$

2.36 From top, acetonide and benzylidene diol protecting groups and the 63 proposed guaiazulene analogue, bottom

2.37 FMOC-Cl, left and an FMOC-protected amine, right 64

2.38 Deprotection mechanism of FMOC with mild base 64

2.39 Postulated deprotection mechanism of a FMOC-analogous azulenebased protecting group $\quad 64$

2.40 The ester $\mathbf{5 5}$ was synthesised and reacted with piperidine/DMF to form 57

2.41 Synthesis of carbamate 59, as presented in the paper by Aumüller et al. 66

2.42 Deprotection of the carbamate with lithium thioethanolate affords 61 and the amine substrate in high yield

2.43 Nitration of the 3-position allows deprotection to take place in much milder conditions

2.44 Base-mediated deprotection of diols

2.45 Proposed mechanism of deprotection $\quad 67$

2.46 Postulated cleavage mechanism of a guaiazulene analogue of $\mathbf{6 2} 68$

2.47 Addition of ethyl formate to the guaiazulenide anion 68

2.48 Synthesis of $\mathbf{6 3}$ using the electrophiles DMF and Vilsmeier reagent 69

2.49 The electrophiles $\mathrm{MeCN}$ and $\mathrm{Ac}_{2} \mathrm{O}$ failed to produce the ketone $66 \quad 70$

2.50 TEMPO-BAIB oxidation of 35 failed to produce the aldehyde $63 \quad 70$

2.51 Attempted Swern oxidation of $\mathbf{3 5}$ resulted in the sulfonium cation $\mathbf{6 7} \quad 70$

2.52 Mechanism of Swern oxidation 71

2.53 Synthesis of the guaiazulene 3-sulfonium cation 68

$\begin{array}{ll}\mathbf{2 . 5 4} \text { Resonance structures of } \mathbf{6 3} \text { upon deprotonation } & 72\end{array}$

2.55 The aldehyde $\mathbf{6 3}$ and its cis- and trans- enol tautomers 72

2.56 General form of a guaiazulene-functionalised silyl ether protecting
group

$\begin{array}{lll}\text { 2.57 } & \text { Synthesis of the trimethylsilyl derivative } 69 & 74\end{array}$

$\begin{array}{lll}\text { 2.58 Proposed route to the silyl ether 70 } & 74\end{array}$

2.59 The positions on the azulene structure on which calculations were performed 76

2.60 $\sigma_{\mathrm{p}}$ vs. absorption wavelength for a series of derivatives at positions 1-6 78

2.61 Absorption wavelength vs position on azulene ring for all derivatives 78

2.62 The ester $\mathbf{3 1}$ and the lactone $\mathbf{4 4}$ exhibit different colours despite their
chemical similarities

2.63 The keto- and enol- tautomers of the aldehyde $\mathbf{6 3} 80$

$\begin{array}{ll}\text { 2.64 Synthesis of a guaiazulene sulfonamide } & 81\end{array}$

2.65 Possible synthetic route to $\mathbf{6 3}$ using a Weinreb amide 82

2.66 Possible two-step synthetic route to $63 \quad 82$ 


\section{List of Tables}

1.1 Effect of substitution on the HOMO-LUMO gap 20

2.1 List of $S_{0} \rightarrow S_{1}$ transition values $(\mathrm{nm})$ for various monosubstituted azulenes at positions 1-6

2.2 Deviation of the $S_{0} \rightarrow S_{1}$ absorbance values from that of azulene

2.3 Comparison of the relative energy of the three tautomers under different conditions and computational techniques 


\section{List of Abbreviations}

HOMO Highest Occupied Molecular Orbital

LUMO Lowest Unoccupied Molecular Orbital

LDA Lithium Diisopropylamide

TLC Thin-Layer Chromatography

EDG Electron-Donating Group

EWG Electron-Withdrawing Group

MO Molecular Orbital

PS-DVB Polystyrene-Divinylbenzene

DMSO Dimethylsulfoxide

NMR Nuclear Magnetic Resonance

HPLC High Performance Liquid Chromatography

FTIR Fourier-Transform Infra-Red

IR Infra-Red

DMF Dimethylformamide

THF Tetrahydrofuran

HMBC Heteronuclear Multiple Bond Coherence

DIPEA Diisopropylethylamine

TEMPO 2,2,6,6-tetramethylpiperidine-1-oxyl

BAIB Bis(acetoxy)iodobenzene

DBU 1,8-Diazabicycloundec-7-ene

DFT Density Functional Theory

TDDFT Time-Dependant Density Functional Theory

RBF Round Bottomed Flask

d Doublet

t Triplet

quin Quintet

sep Septuplet

m Multiplet

UV-Vis Ultraviolet - visible 


\section{Chapter 1.}

\section{Introduction}

In this project, the physical and chemical properties of azulene derivatives are investigated for the purpose of creating self-indicating chromophores, in particular for use as coloured protecting groups and self-indicating solid-phase resins. By exploiting the physical properties and unique reactivity of the azulene moiety, we hoped to create products of novel utility and function.

\subsection{Azulenes}

Azulene (1) is a compound that has long held interest within physical organic chemistry, due to the structural and electronic characteristics that give rise to its unique optical properties and reactivity. In particular, azulene has a dipole moment of 1 debye $^{1}$, exhibits an intense blue colour and is the smallest known hydrocarbon to do so. ${ }^{2}$

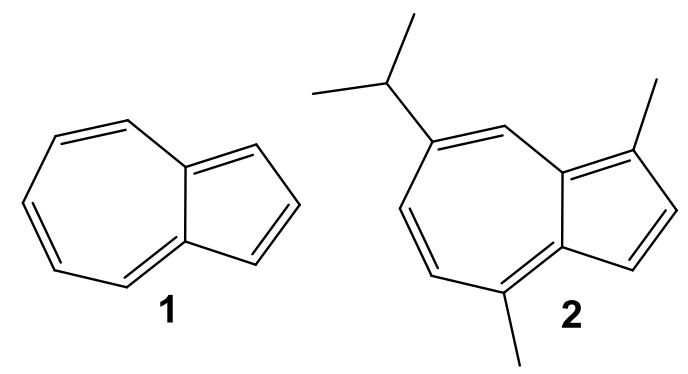

Figure 1.1 The chemical structures of azulene 1 and guaiazulene $\mathbf{2}$

The presence of a blue colour has been observed in distillations of various oils for the past 500 years. $^{3}$ Originally this colour was thought to be due to contamination from the copper apparatuses used during distillation, but it was later shown that this blue colour was volatile and soluble in ethers. In 1864 this blue oil was given the name 'azulene', which today refers both to the parent compound and the class of derivatives thereof. In 1915, an azulene was finally isolated and the molecular formula was found to be $\mathrm{C}_{15} \mathrm{H}_{18}$. Several suggestions were given for its structure, including tricyclic compounds and benzofulvenes, but hydrogenation over platinum revealed that the basic structure was bicyclic and the hydrogenated products shared similarities with 
some sesquiterpenes. In 1936 a 5,7 ring structure was proposed after observing the isomerisation of various azulenes to naphthalenes at high temperatures, and this was confirmed by synthesis the next year. ${ }^{3}$

The parent compound azulene is a fully conjugated bicyclic hydrocarbon, with a fused 5,7 ring system. While this compound is an isomer of naphthalene, its properties are remarkably different, owing to the consequences of this 5,7 ring arrangement. ${ }^{2}$

\subsubsection{Resonance structure of azulene}

Hückel's rule predicts that conjugated rings with a number of $\pi$-electrons equal to $4 n+2$ where $n$ is an integer (i.e. no. of $\pi$-electrons $=2,6,10,14$ and so on) will exhibit aromatic stabilisation. For conjugated rings with an odd number of $\pi$ electrons, this results in the existence of unusually stable carbocations and carbanions, as in the case of the cyclopentadienyl anion and the tropylium cation (Figure 1.2).

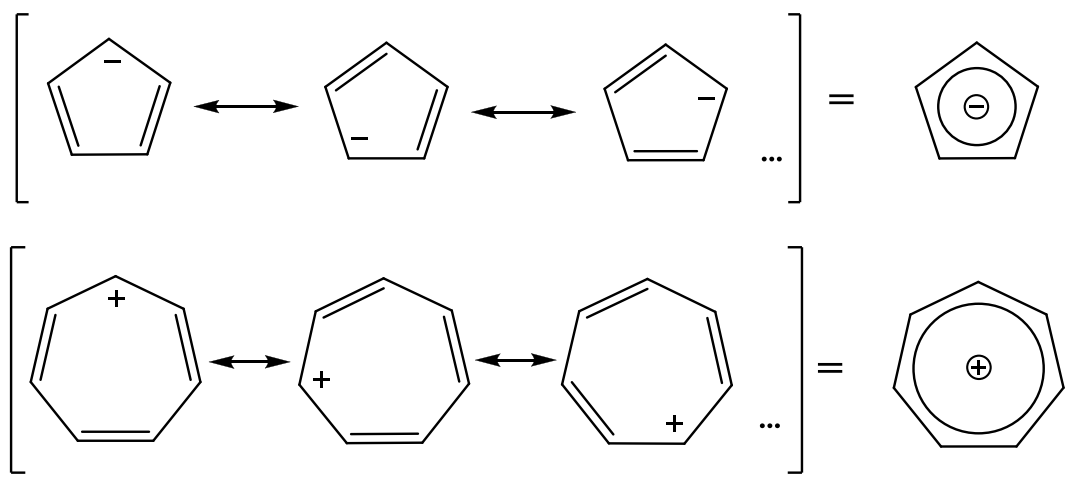

Figure 1.2 Resonance and mesomeric forms of the aromatic ions cyclopentadienide, top and tropylium, bottom

By considering Hückel's rules in the case of azulene, a possible electronic structure consisting of a cyclopentadienide ring fused to a tropilium ring becomes apparent. The dipole moment of azulene can then be explained by considering both the neutral and zwitterionic structure as being in resonance with each other (Figure 1.3). ${ }^{4}$

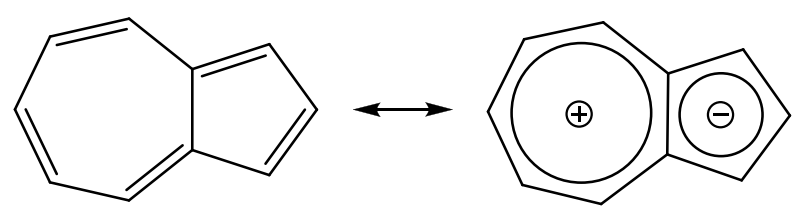

Figure 1.3 Mesomeric forms of azulene. 


\subsection{Colour of azulenes}

Azulene absorbs strongly in the visible light region between 500 and $700 \mathrm{~nm}^{5}$ and is transparent only to blue light in the $400 \mathrm{~nm}$ region and a small amount of light over $700 \mathrm{~nm}$ (Figure 1.4), resulting in an intense blue colouration. This suggests an unusually small HOMO-LUMO gap, in the order of $1.8 \mathrm{eV}$.

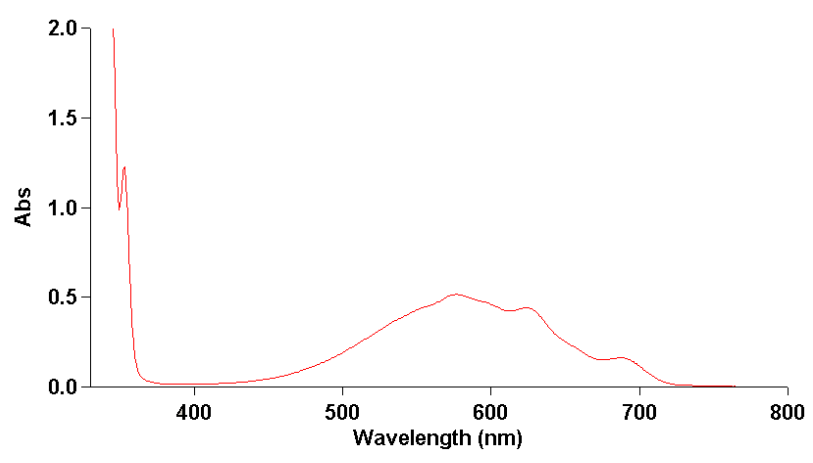

Figure 1.4 UV-Vis spectrum of azulene

\subsubsection{Non-alternant hydrocarbons}

Despite the level of conjugation in azulene, its intense blue colour is not a direct result of conjugation. If it were, one would expect its isomer, naphthalene, to also be highly coloured, which is not the case. The basis of azulene's low HOMO-LUMO gap lies in the poor overlap between the HOMO and LUMO orbitals. ${ }^{5}$

Azulene's colour arises ultimately from the fact that it is a non-alternant hydrocarbon. ${ }^{2,6}$ The defining feature of a non-alternant hydrocarbon (in comparison to an alternant hydrocarbon) is the pattern in which the carbon atoms in the rings are arranged. The original definition of a non-alternant carbon states that "A conjugated unsaturated hydrocarbon is non-alternant if the carbon atoms cannot be divided into two groups such that all the atoms of the first group have only atoms of the second group as immediate neighbours, and vice versa".

In order to determine whether or not a hydrocarbon is non-alternant, one can follow the following steps: Starting at an arbitrary position, move around the conjugated structure, labelling every second carbon. If once every second carbon is labelled there exist two adjacent labelled or unlabelled carbons, the system is non-alternant. Otherwise if there are no labelled or unlabelled carbon atoms adjacent to each other, it is an alternant hydrocarbon. In a cyclic compound, the presence of an odd-membered 
ring will ensure the hydrocarbon is non-alternant. Examples of alternant and nonalternant hydrocarbons are given in Figure 1.5.

Alternant<smiles>C=C/C=C/C=C</smiles><smiles>c1ccc2ccccc2c1</smiles>

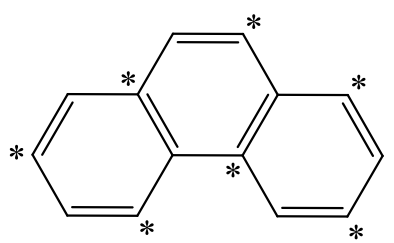

Non-alternant
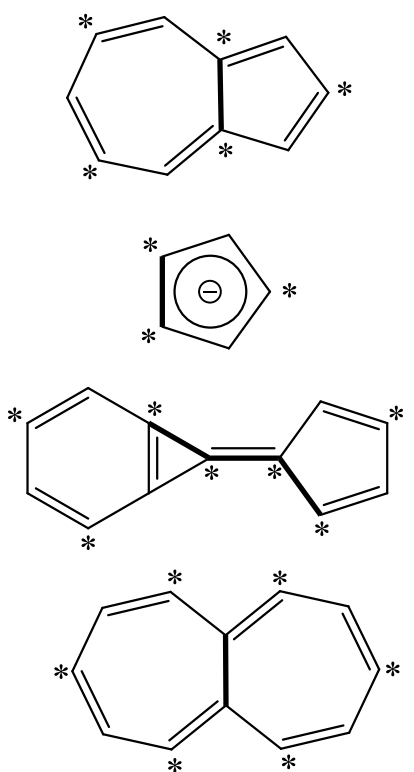

Figure 1.5 Examples of alternant and non-alternant hydrocarbons

The difference between alternant and non-alternant hydrocarbons manifests itself in the way the $\pi$-orbitals are arranged within the molecule. In general, the arrangement of the molecular $\pi$-orbitals are relatively easy to predict in an alternant hydrocarbon, ${ }^{7}$ when compared to a non-alternant hydrocarbon. For instance, for any given alternant hydrocarbon the highest energy $\pi$ molecular orbital will necessarily have a node between each adjacent carbon - the phase essentially alternates in the same pattern as the labelled alternant hydrocarbons in Figure 1.5.

In a non-alternant hydrocarbon, the $\pi$ orbitals can be more difficult to assign than their alternant counterparts. Perhaps the most illustrative example can be found comparing the LUMO+4 orbital (the highest energy $\pi$ orbital) for both naphthalene and azulene (Figure 1.6). The LUMO+4 of naphthalene adopts an opposite phase on every carbon. In azulene however, it is not possible to arrange the atomic orbitals in this way, so a node inevitably lies on two of the carbons in the wavefunction.

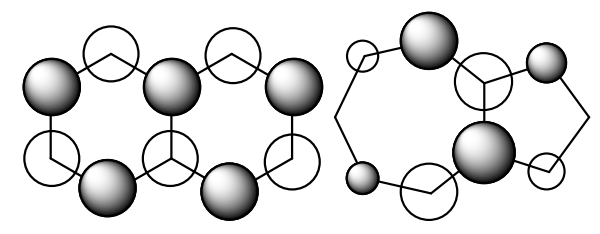

Figure 1.6 LUMO+4 for naphthalene, left and azulene, right 
Due to this asymmetry, the HOMO and LUMO orbitals often inhabit different areas of the molecule in non-alternant hydrocarbons, resulting in a poor overlap between the two orbitals. This usually results in a small separation of charge and hence a small dipole moment to occur within the molecule, a typical characteristic of non-alternant hydrocarbons. ${ }^{7}$

Consider the HOMO and LUMO orbitals of both naphthalene and azulene, illustrated in Figure 1.7.

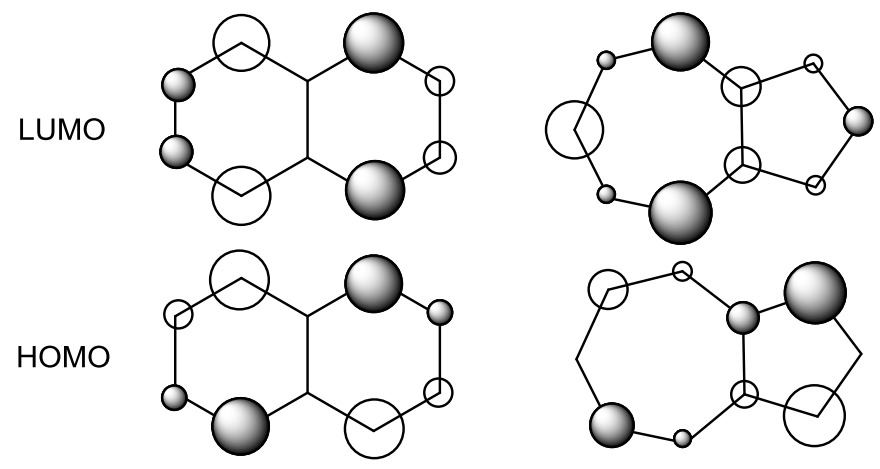

Figure 1.7 The HOMO and LUMO of naphthalene, left, and azulene, right

The HOMO and LUMO of naphthalene have a high degree of overlap. Ignoring the differences in phase and considering the electron density only, the coefficients of the atomic orbitals in the HOMO and LUMO of naphthalene match up closely. The HOMO and LUMO orbitals of azulene, however, exhibit little overlap. Several regions of high electron density in the HOMO are nodes in the LUMO and vice-versa. There is experimental evidence for this poor overlap - the major mode of fluorescence in azulene occurs between the ground state and the second excited state ${ }^{8}$, indicating a much better overlap between the HOMO and LUMO+1 orbitals than between the HOMO and LUMO.

\subsubsection{Origin of the small HOMO-LUMO gap}

The poor overlap between HOMO and LUMO wavefunctions results in a reduced separation between the energies of the HOMO and LUMO orbitals, ${ }^{2}$ resulting in absorption of red in the visible portion of the electromagnetic spectrum. This is a consequence of electron correlation, as the energy of each orbital is influenced by not only all other occupied orbitals, but also by all unoccupied orbitals to varying degrees depending on orbital overlap. 


\subsubsection{Colour in azulene derivatives}

Substituents located on the azulene backbone can influence the colour of the derivatives in profound ways. ${ }^{2}$ Due to the non-alternant nature of azulene, substituents have different effects on the electronic structure depending on whether they are placed on odd or even numbered positions. ${ }^{6,8}$ The numbering system used for all azulene derivatives in this project is presented in Figure 1.8.

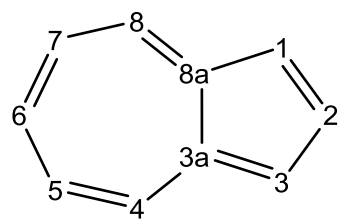

Figure 1.8 Numbering system of the azulene moiety

In the series of monosubstituted methyl azulene derivatives, it has long been known ${ }^{3}$ that the visible light absorption peak undergoes a bathochromic shift (shift towards longer wavelengths) in the compounds 1-methylazulene and 5-methylazulene and a hypsochromic shift (shift towards shorter wavelengths) in 2-methyl, 4-methyl and 6methylazulene. The bathochromic shift of the absorption in 1- and 5- methylazulenes results both in less red light being transmitted, and more blue light being transmitted, giving the compound a slightly bluer colour. This is consistent with the observed colour difference between azulene and guaiazulene (2) - because guaiazulene has two alkyl groups on odd-numbered positions and one on an even-numbered position there is an overall shift to a longer wavelength absorbance and a corresponding shift in colour from indigo to blue. ${ }^{8}$

Alkyl groups are mildly electron donating in aromatic $\pi$ systems, and produce correspondingly mild changes in colour. Much more dramatic colour changes occur when stronger electron donating groups or electron withdrawing groups are used, which often causes a shift in absorption that results in colours ranging from green to orange-red.

This phenomenon can be explained by the influence the substituents have on the valence orbitals of azulene. In Figure 1.9, the effect of an electron donating group (EDG) or an electron withdrawing group (EWG) at the 1 position on the HOMO, LUMO and LUMO+1 orbitals is illustrated. 

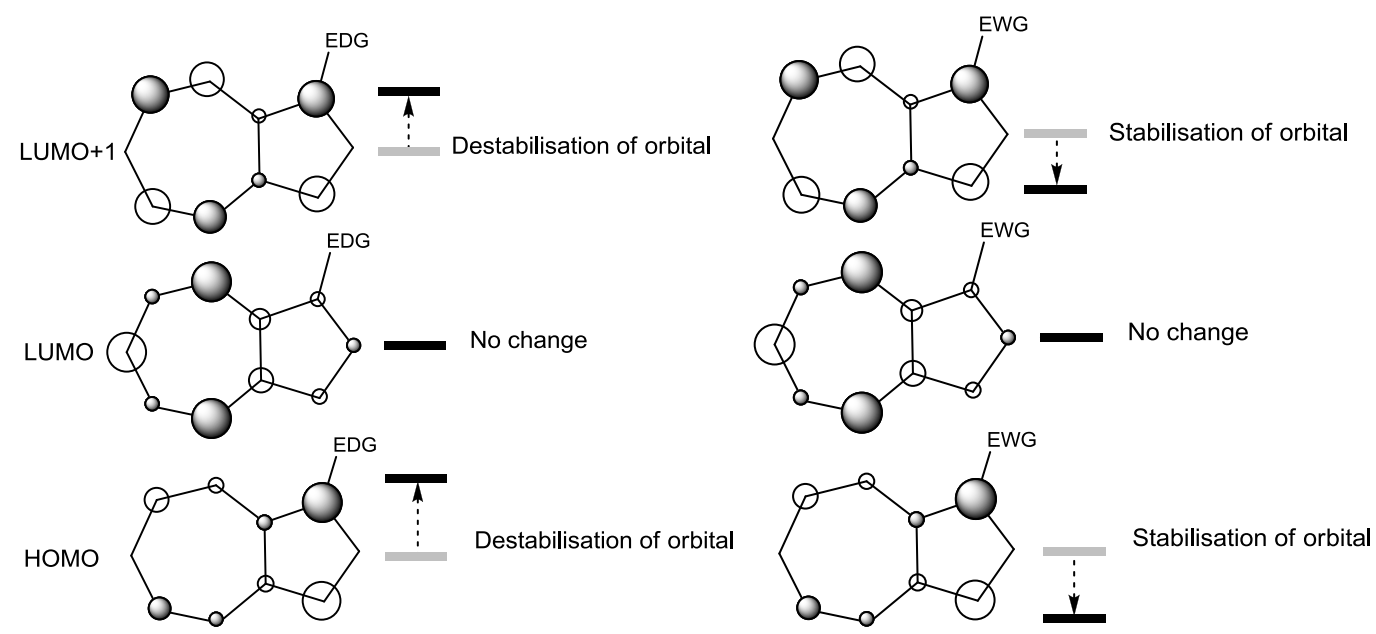

Figure 1.9 The effect of EDGs and EWGs at the 1- position on the electronic structure of azulene

The EDG at the 1 position has the effect of destabilising the HOMO and LUMO+1 orbitals by donating electron density to a part of the molecule that is already electron rich. An EWG at the same position has the opposite effect; electron density is withdrawn from the electron rich areas of the $\mathrm{HOMO}$ and $\mathrm{LUMO}+1$, resulting in a stabilisation of these two orbitals. In both cases the LUMO is not affected to any significant degree, as there is very little electron density at the 1 position of the LUMO. Overall, electron donating groups at the 1 position cause a net decrease in the HOMO-LUMO gap, and consequently a bathochromic shift of the absorption band of visible light. Conversely, electron withdrawing groups at the 1 position cause a net increase of the HOMO-LUMO gap which results in a hypsochromic shift of the absorption band. Substitution at the 5 position also produces these effects, but to a lesser extent.

The situation is reversed when the substitution is at the 2,4 or 6 positions.

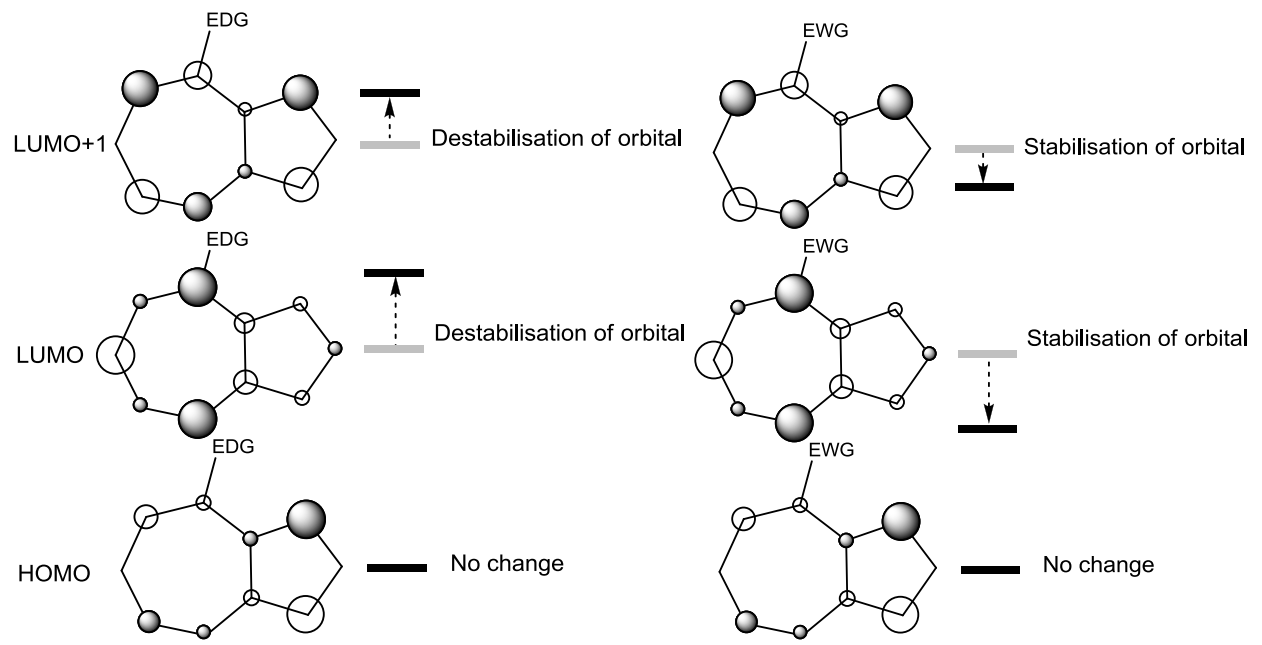

Figure 1.10 The effect of EDGs and EWGs at the 4- position on the electronic structure of azulene 
In this case, the LUMO experiences the largest change in energy from the substituted position, with an EDG widening the gap and an EWG narrowing it. The effect of the EDG or EWG at the 4 or 6 positions on the HOMO-LUMO gap is therefore opposite to that of an EDG or EWG at the 1 position. Substitution at the 2 position also exhibits these effects, but to a lesser extent.

The effects of EDG and EWG placement on the HOMO-LUMO gap can be summarised in Table 1.1:

\begin{tabular}{l|l|l} 
& $\begin{array}{l}\text { Substitution at an odd- } \\
\text { numbered position }\end{array}$ & $\begin{array}{l}\text { Substitution at even- } \\
\text { numbered position }\end{array}$ \\
\hline EDG & $\begin{array}{l}\text { Lower gap energy/ } \\
\text { longer wavelength } \\
\text { absorption }\end{array}$ & $\begin{array}{l}\text { Higher gap energy/ } \\
\text { shorter wavelength } \\
\text { absorption }\end{array}$ \\
\hline EWG & $\begin{array}{l}\text { Higher gap energy/ } \\
\text { shorter wavelength } \\
\text { absorption }\end{array}$ & $\begin{array}{l}\text { Lower gap energy/ } \\
\text { longer wavelength } \\
\text { absorption } \\
\text { Table 1.1 Effect of substitution on the HOMO-LUMO gap }\end{array}$
\end{tabular}

Substituents such as carbonyl groups and halogens can generate quite substantial colour changes through substitution of the azulene ring., ${ }^{8,2,6}$ For example, while azulene is blue, 1-formylazulene is crimson in colour, and 1,3-diformylazulene is red. 6-formylazulene on the other hand is blue-green, and 1,3-difluoroazulene is green.

\subsection{Reactivity of azulenes}

The structural and electronic properties of azulene give rise to some unusual and varied reactivity. Like most aromatic compounds, azulene primarily undergoes electrophilic aromatic substitution, although nucleophilic aromatic substitution is also possible. Unlike most aromatic compounds however, azulene reacts much more readily with electrophiles and nucleophiles, demonstrable by the fact that aromatic substitution reactions involving azulene often proceed without the presence of a Lewis acid or other catalyst. In particular, the moderately nucleophilic 1 and 3 positions readily undergo electrophilic aromatic substitution, ${ }^{9}$ whereas the 4,6 and 8 positions are electrophilic, making them susceptible to nucleophilic substitution. ${ }^{10}$ In contrast, the 2, 5 and 7 positions are much less reactive to either nucleophilic or 
electrophilic attack, only experiencing substitution under harsher conditions when the more reactive sites are already substituted. ${ }^{11}$

This pattern of reactivity can be explained in several ways. A valence bond approach considers resonance forms when a charge separation occurs. As it turns out, the generation of a charge in certain specific positions will allow an aromaticallystabilised countercharge to be developed. It is these positions that tend to be more reactive towards nucleophiles or electrophiles.

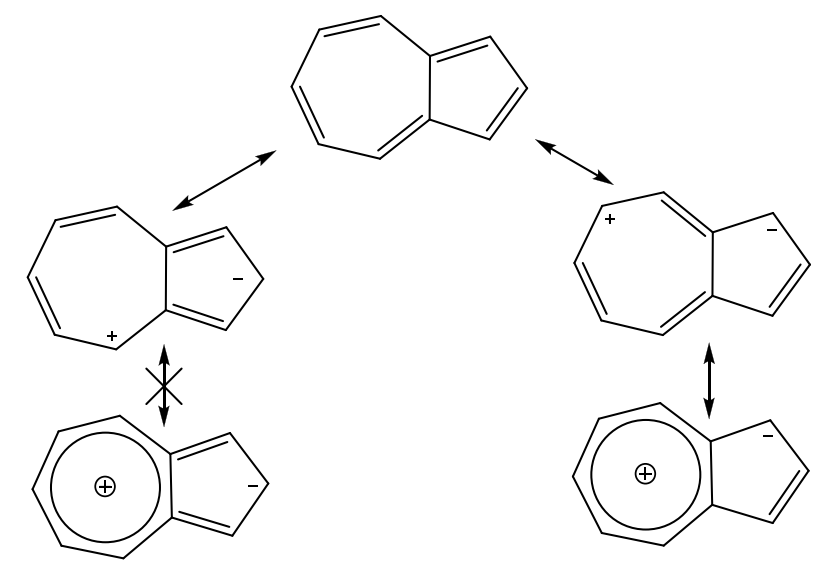

Figure 1.11 Aromatically-stabilised and non aromatically-stabilised charge separated resonance forms of azulene

This is illustrated in Figure 1.11. Placement of the negative charge at the 1- or 3position allows access to the tropylium-stabilised resonance structure, whereas placement of the negative charge at the 2- position prohibits access to this tropyliumstabilised resonance structure, as it requires the existence of pentavalent carbon atoms. As the charge-separated resonance structure involving the tropylium is afforded extra stability, this resonance structure will dominate, leading to a partial negative charge on the 1- and 3- positions. Thus, a resonance-structure approach is sufficient to explain the greater reactivity that these positions have towards electrophiles.

Not only can this line of reasoning be applied to the reactivity of the azulene starting material, it can also be used to explain differences in energy - and hence reactivity between reactive intermediates. As a consequence of the Hammond postulate, the relative energy of a transition state can be approximated by assessing the stability of the nearest reactive intermediate. In this way, the relative rates of different reaction mechanisms can be rationalised through the comparison of these intermediates. 


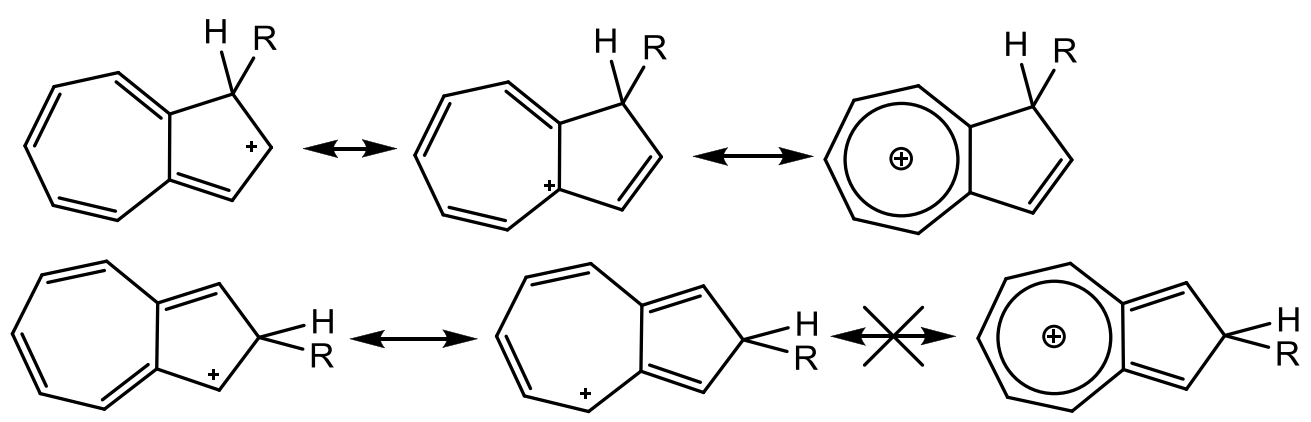

Figure 1.12 Resonance structures of the 1-addition intermediate, above, and of the 2-addition intermediate, below

In Figure 1.12, the reactive intermediate formed by addition of $R$ (an electrophile) to the 1- position has access to the tropylium resonance structure, whereas the intermediate formed by addition to the 2- position does not. This suggests the 1addition intermediate is lower in energy than the 2-addition intermediate, which indicates that attack at the 1 - position is the preferred reaction pathway in electrophilic substitution.

This reasoning can be further expanded to explain the reactivity of azulene towards nucleophiles. This is illustrated in Figure 1.13 in which the resonance structures with the positive charge in the 4- or 6- positions have the stabilising contribution of the cyclopentadienyl anion, whereas resonance structures involving the positive charge at the 5- position do not.

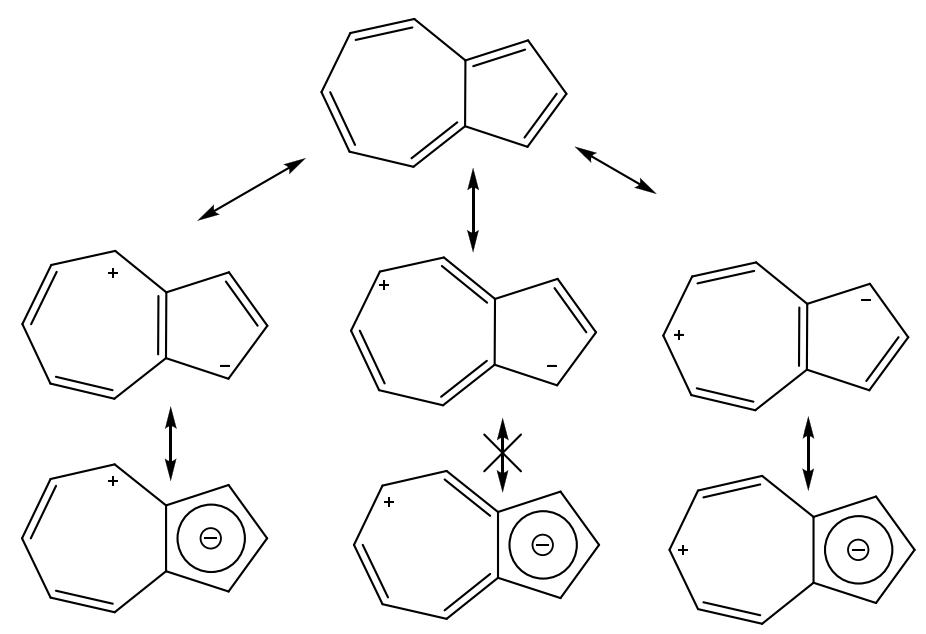

Figure 1.13 Charge-separated resonance forms of azulene, illustrating the accessibility of the stabilising cyclopentadienide form

This valence bond approach gives a good qualitative argument as to why azulene exhibits the pattern of reactivity that it does. However to gain a more complete picture of this reactivity (and also the reactivity of azulene derivatives), it is necessary to return to the results of MO theory. 
The aromatic molecule acts as the nucleophile in electrophilic aromatic substitution, which means that the principal frontier orbital involved in the reaction is the HOMO. As can be seen in Figure 1.14, the electron density is greatest on the 1- and 3positions in the HOMO, facilitating electrophilic attack of these two positions over all others.

Similarly, in nucleophilic aromatic substitution, the LUMO of the aromatic ring is the MO that is involved in the reaction. As before, the wavefunction is concentrated on the 4-, 6- and 8- positions on azulene.

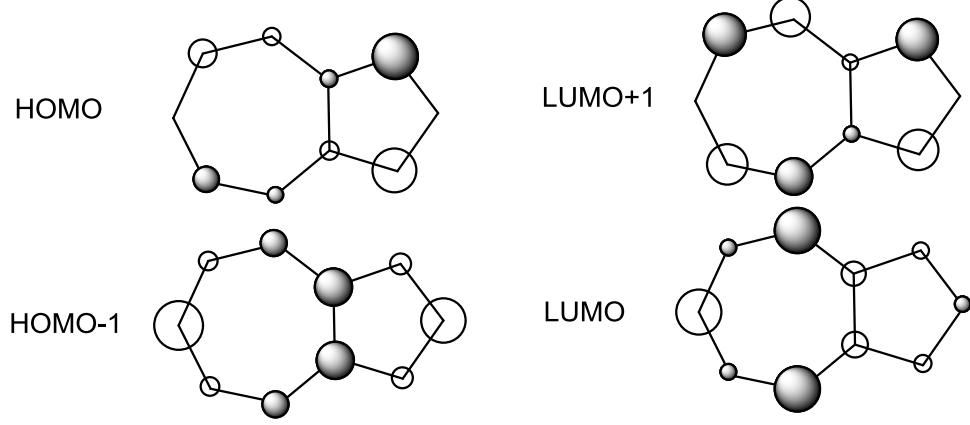

Figure 1.14 The frontier molecular orbitals of azulene

From this, it becomes clear why the 2-, 5- and 7- positions on azulene exhibit low reactivity. Electrophilic attack on the 2- position would require access to the HOMO1 orbital, whereas nucleophilic attack on the 5- and 7- positions requires access to the LUMO+1 orbital. The energy differences between the HOMO-1 and HOMO and between LUMO and LUMO+1 appear large enough to prevent reactions occurring at the 2-, 5- and 7-positions on unsubstituted azulene to any significant degree.

\subsection{Common reactions involving azulene}

There currently exists a wealth of information on the synthesis of azulene derivatives. Much of this work was carried out by Klaus Hafner and co-workers between 1955 and 1962.

\subsubsection{Reactions at the 1 and 3 positions}

Many reactions at the 1 and 3 positions of azulene are analogous to electrophilic aromatic substitutions on benzene, although most reactions are facile enough to not 
require Lewis acid catalysis, owing to the increased nucleophilicity of the 1- and 3positions on the azulene moiety as compared with benzene.

Formylation can be achieved through the Vilsmeier-Haack reaction ${ }^{12}$, which involves the generation of Vilsmeier-reagent (a chloroiminium species) through addition of phosphorus oxychloride to dimethylformamide, which is then added to the azulene substrate followed by addition of aqueous sodium hydroxide. This reaction is extremely facile and can give quantitative yields. On azulene itself, formation of the dialdehyde $\mathbf{4}$ as a minor product usually occurs alongside the major aldehyde product 3, as depicted in Figure 1.15.

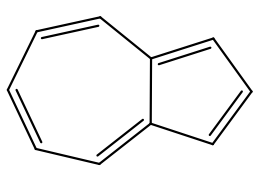

1

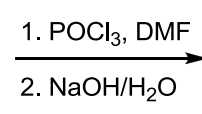

-

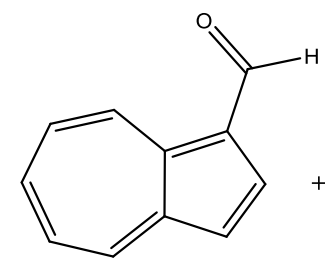

3

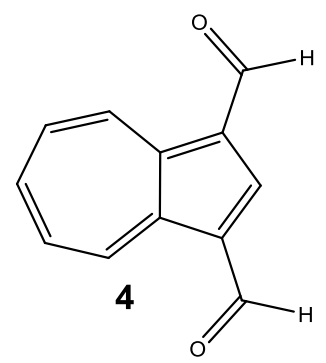

Figure 1.15 Reaction scheme of the Vilsmeier-Haack reaction on azulene

Friedel-Crafts acylation-type reactions are also very facile on the azulene moiety (Figure 1.15), producing ketones such as $\mathbf{5}$, while not requiring Lewis acid activation to proceed. $^{13}$

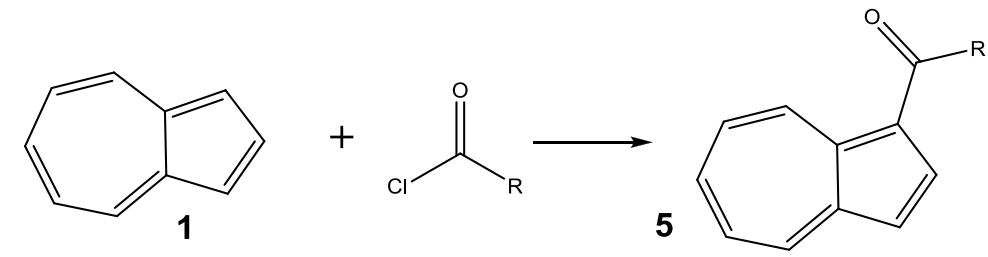

Figure 1.16 Acylation of azulene

Somewhat surprisingly, analogous Friedel-Crafts alkylation reactions on azulene have not been successfully performed in acceptable yields. A paper by A. G. Anderson et al. details this effort. Alkylation on azulene was attempted using a variety of alkyl and aryl halides and Lewis acids, resulting usually in only circumstantial evidence for the substitution of azulene. The highest yield reported from any reaction was $5.6 \%$ following the usage of benzyl chloride as the substrate and tin tetrachloride as the Lewis acid. ${ }^{14}$ There were no reasons proposed for this lack of reactivity, although comparisons with the poor reactivity of alkyl halides towards thiophene and naphthalene were made. 


\subsubsection{Reactions on the 4,6 and 8 positions}

As mentioned previously, the 4,6 and 8 positions are susceptible to nucleophilic attack. Alkylating these positions is usually done with an alkyllithium or Grignard reagent in two steps, as depicted in Figure 1.17. ${ }^{15}$

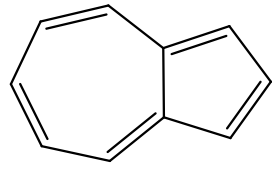

1

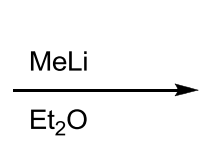

$\mathrm{Et}_{2} \mathrm{O}$

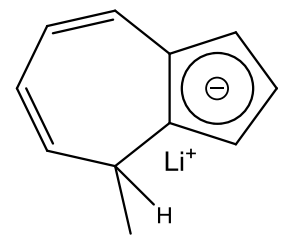

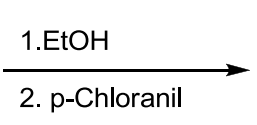

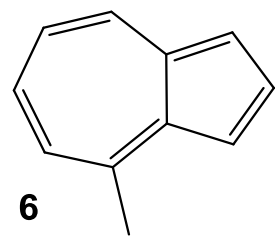

Figure 1.17 Nucleophilic substitution of azulene

Due to the two-step nature of this reaction, only one position can be alkylated at a time. This reaction also preferentially alkylates the 4 and 8 positions, the 6 position only being alkylated after the other two positions are substituted.

4-substituted azulenes such as $\mathbf{6}$ can undergo further reactions such as deprotonation, which can be achieved by bases such as LDA due to the resonance-stabilised form of the anion. An example of deprotonation followed by alkylation using an alkyl halide is given in Figure 1.18. ${ }^{16}$
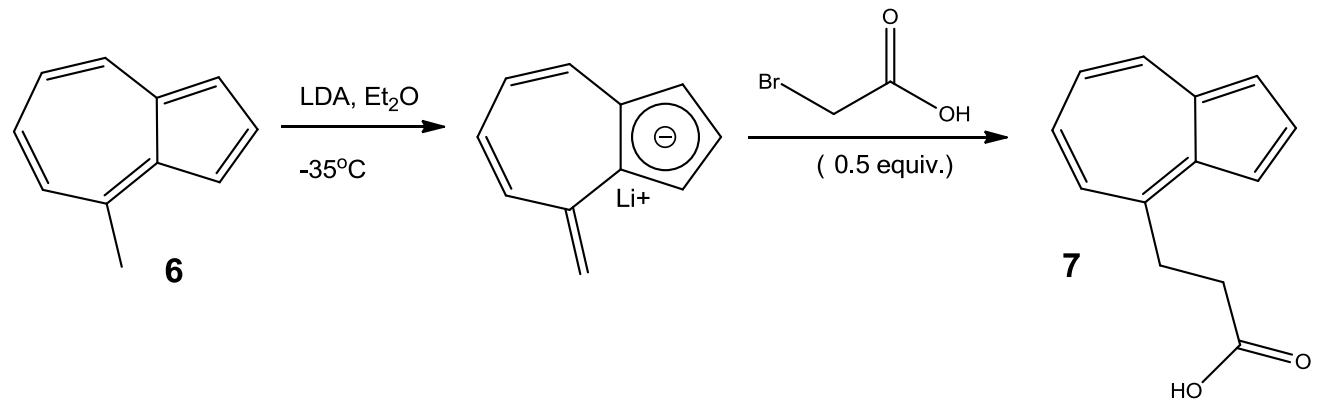

Figure 1.18 Coupling of 4-methyl azulene with bromoacetic acid

\subsubsection{Other reactions involving azulene}

Azulene and its derivatives can be synthesised through a variety of means. There exist many synthetic routes in the literature, some of which have been adopted into undergraduate laboratory courses. One method reported by Lemal et al. involves a $[4 \pi+6 \pi]$ cycloaddition between a fulvene and thiophene dioxide as the final step (Figure 1.19). ${ }^{17}$ 


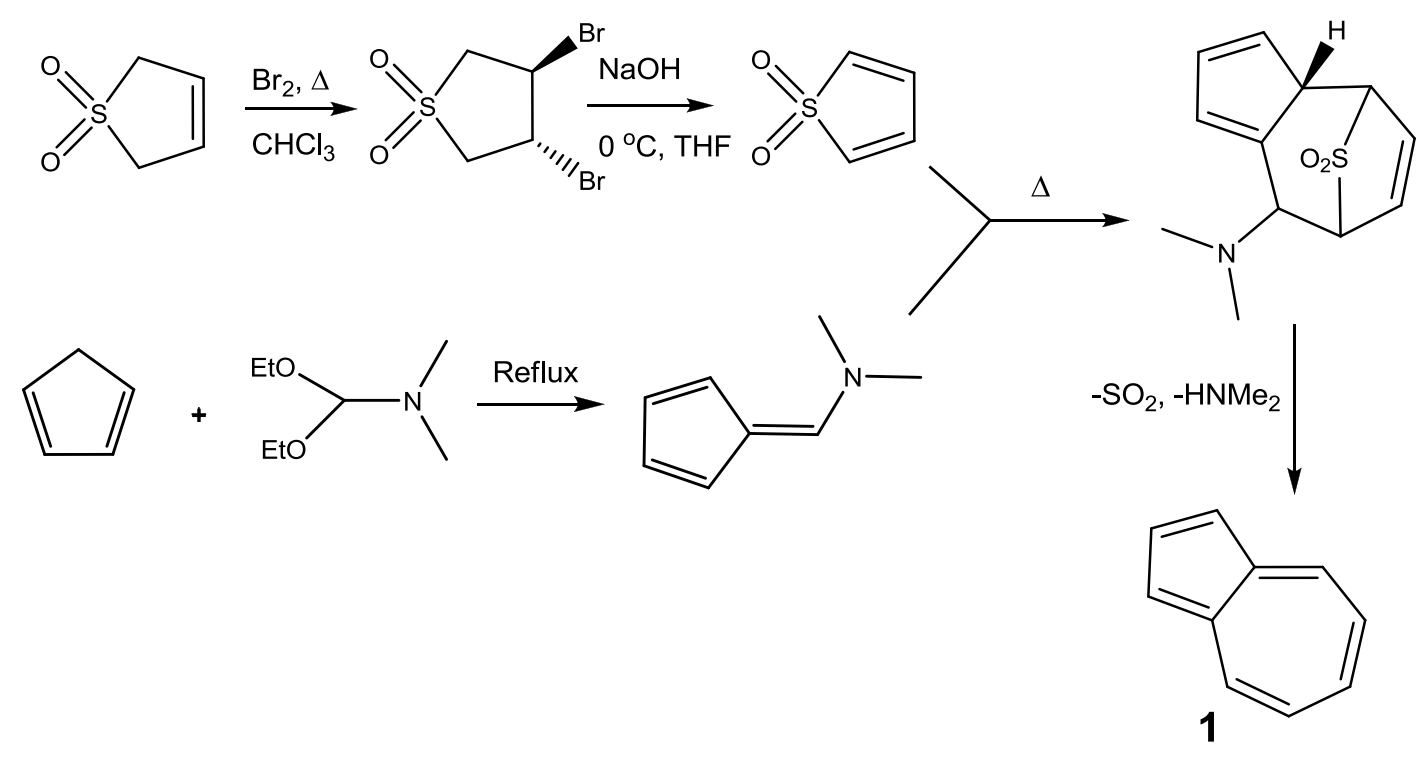

Figure 1.19 4-step synthesis of azulene from cyclopentadiene, dimethylformamide diethylacetal and butadiene sulfone

Some azulene syntheses can be adapted to produce azulene derivatives with substitutions at positions that are otherwise difficult to substitute. An example, first reported by K. Hafner and co-workers ${ }^{18}$ is given in Figure 1.20.

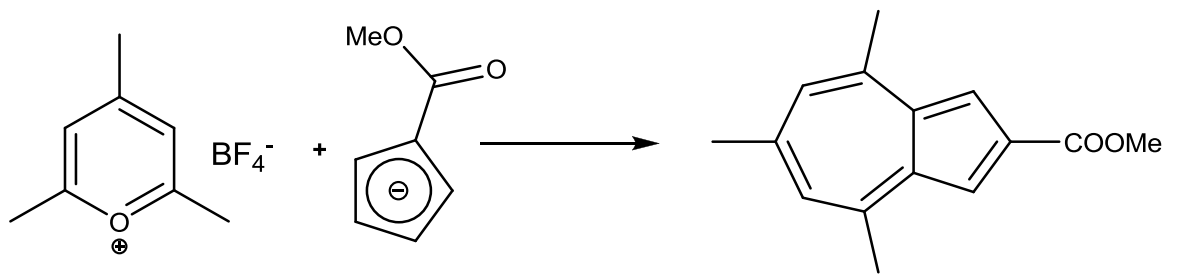

Figure 1.20 Synthesis of 4,6,8-trimethyl azulene 2-methyl ester

In this synthesis, the azulene derivative is created with the 2-,4-,6-, and 8- positions already substituted. The ester at the 2- position in particular is difficult to install on azulene itself.

The azulene analogue of triphenylmethane, tris(azulenyl)methane $\mathbf{8}$ was first reported by $\mathrm{K}$. Hafner ${ }^{19}$ and co-workers, and the most recent synthesis by $\mathrm{S}$. Ito ${ }^{20}$ et al. is a one-step procedure using azulene and the aldehyde 3 (Figure 1.21).

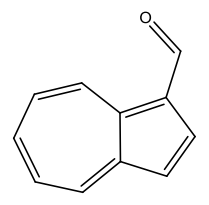

3

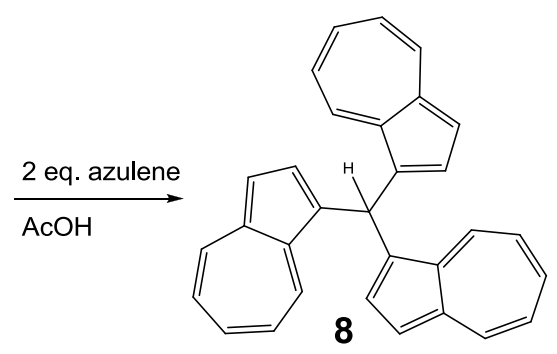

8

Figure 1.21 Synthesis of tris(azulenyl)methane from azulene and 1-formyl azulene, as reported by S. Ito $^{20}$ 


\subsubsection{Current research and applications of azulenes}

A popular area of investigation within azulene chemistry is in creating compounds with novel redox properties, often taking advantage of the fact that azulene can act both as an electron donor or acceptor in a conjugated system, depending on which position the ring is substituted at. Shunji Ito and co-workers are prominent in this area, as their research involves the creation of organic polyelectrochromic materials whose colours vary according to redox state..$^{21,22}$

At the commencement of this project, only one paper had been found reporting the use of an azulene derivative as a protecting group. The following procedure was reported by Ingo Aumüller and Thisbe K. Lindhorst in $2006^{16}$ in which a guaiazulene derivative was attached onto a carbohydrate hydroxyl to form a chromophoric monosaccharide (Figure 1.22), in essence creating a chromophoric protecting group.

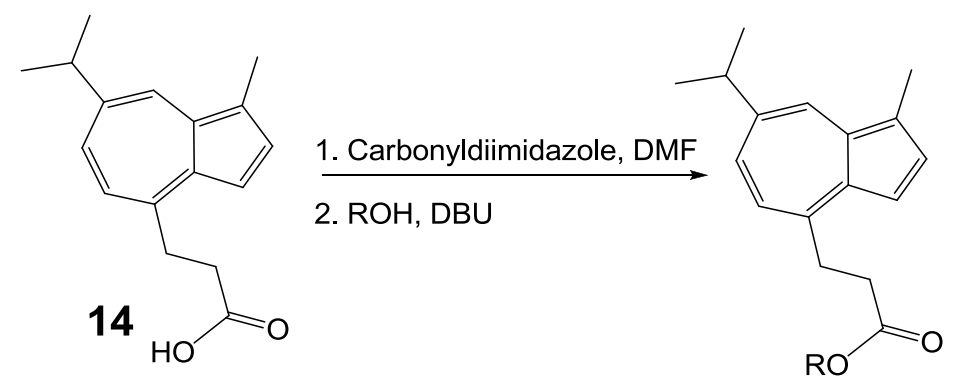

Figure 1.22 Protection of an alcohol using the carboxylic acid $\mathbf{1 4}$

A key point in this paper is that it mentions the ease with which compounds can be purified by column chromatography due to the ability to visualise the compound's progress through the column. This allowed them to easily perform several parallel purifications by column chromatography on a range of guaiazulene-functionalised monosaccharides. 


\subsection{Project aims}

The main aims of this research involve the investigation of the reactivity of certain azulene derivatives for the purposes of creating products of novel and useful function, by utilising the unique physical properties (colour and dipole moment) and chemical reactivity of the azulene moiety.

Owing to the relative novelty of this project (in that there are very few examples in the literature of similar research), the approach taken to the research was primarily an exploratory one. As such, there were several paths of inquiry during this research that received attention to varying degrees. Of these, two main areas of investigation predominated.

The first investigation was concerned with creating azulene derivatives for use as chromophoric tags and protecting group systems, followed by consideration of the conditions involved to add and remove these chromophoric groups.

The second investigation was concerned with creating polystyrene-divinylbenzene resin-bound azulene derivatives, with the possibility of applications such as scavenger resins.

\subsection{Azulene derivatives as coloured tags and protecting groups}

Protecting groups are a ubiquitous part of organic chemistry, as their use allows chemists to control reactivity and selectivity in their chemical systems at the expense of a couple of synthetic steps. Although there are several examples of syntheses performed without the use of protecting groups, ${ }^{23}$ these are presently an exception rather than the rule. Considering the current level of synthetic knowledge, it could be said that the benefits of the use of protecting groups in terms of selectivity outweigh the loss in yield associated with the extra protection and deprotection step for most chemical systems at present.

There are two basic requirements of a protecting group. ${ }^{24}$ The first is that it must be relatively simple to attach and cleave, preferably under mild conditions. The second is 
that it exhibits orthogonality towards other protecting and deprotecting conditions. The protecting group must also at the very least be partially stable towards a range of other common reaction conditions, such as acidic, basic, oxidising and reducing conditions, and be stable towards nucleophiles and electrophiles, not just in terms of deprotection but also in terms of side reactions on the group itself - the protecting group itself must not easily participate in reactions that render it non-functional.

\subsubsection{Chromophoric tags}

The introduction of chromophores into a molecule provides instant feedback of information through visual inspection. This allows reactions to be qualitatively monitored visually, and also allows purification steps such as phase separation and column chromatography to be performed without the need for analysis of all phases and fractions by TLC.

Chromophoric tags can be divided into three broad groups. The first are UV-active chromophores, of which the benzyl protecting group is a well-known example. The benzyl protecting group is widely used in organic synthesis, and one of its notable features is the absorption of light at around $250 \mathrm{~nm}$, allowing the compound to appear as a dark spot upon UV-irradiation of fluorophore-doped TLC plates. ${ }^{25}$

Fluorescent chromophores are another class of chromophoric tag that emit visible light upon irradiation with ultraviolet light, thus allowing them to be visualised on TLC plates and in column chromatography through the use of a UV-lamp. While fluorescent chromophores such as fluorescein ${ }^{26}$ and the dansyl group ${ }^{27}$ (Figure 1.23) mainly see use in molecular biological applications such as protein labelling, there are examples of their use in organic chemistry. For instance, $O$-dansyl groups have been used as a fluorescent leaving group in solid-phase synthesis, allowing monitoring of the nucleophilic substitution of these groups through UV-irradiation.
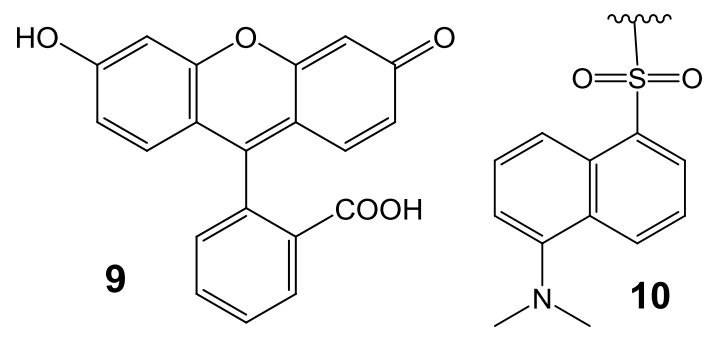

Figure 1.23 Fluorescein (9) and the dansyl group (10) are examples of groups commonly used as fluorescent tags 
The third group, and the type that is the focus of this research, is that of coloured protecting groups. Examples of coloured protecting groups are rare in the literature, and outside of azulene-based moieties only one example is known to the author, which is of an azo dye-based protecting group for amines (Figure 1.24). ${ }^{28}$

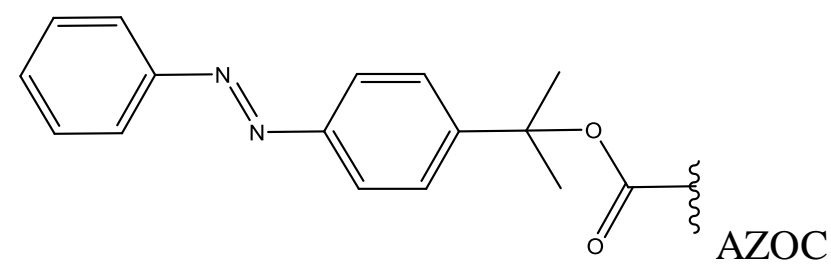

Figure 1.24 The orange-red ( $p$-phenylazophenyl)isopropyloxycarbonyl (AZOC) protecting group

The presence of any sort of visual identifier such as a bound chromophore not only allows faster visual feedback during reactions and purification, but also reduces the need for destructive analytical techniques such as TLC staining.

\subsubsection{General strategy of azulene protecting group research}

Much of the effort expended throughout this project was towards the development of a functional protecting group. The general approach was to identify azulene derivatives with interesting or unusual chemistry, propose common functional groups that could be protected and deprotected through this chemistry, and investigate these conditions using a suitably functionalised small molecule. This is in contrast to a functional group centred approach, wherein certain functional groups would be chosen and appropriate azulene-based protecting group chemistry be developed for that particular protecting group. This approach was not always followed, but for the most part the research pursued this method of inquiry.

Another important design aspect to note is that there is a choice between having the functional group connected electronically (through resonance either connected directly or with a conjugated linker), or isolated from the azulene ring (connected through a non-conjugated linker, such as an alkyl chain) (Figure 1.25). In the former case, the azulene moiety directly affects the chemistry of the functional group and vice versa. This means not only that the reactivity and the protection-deprotection conditions of the functional group are altered from their usual properties, but a colour change upon protection/deprotection is likely. Conversely, in the latter case the azulene moiety essentially acts as a spectator - it does not influence the electronics of 
the bound functional group, and it does not give a colour change upon protection/deprotection. Due to this difference, a protecting group of the former type has much more potential to be a novel protecting group with unique protecting/deprotection conditions and an associated colour change, whereas the latter merely has novelty value as a protecting group with an associated colour. The payoff of course, is that when the protection/cleavage conditions for a given protecting group system is investigated and optimised, the conditions of the latter type - whose properties would resemble existing protecting groups to a much greater degree than the former - were expected to take much less effort to fully investigate.
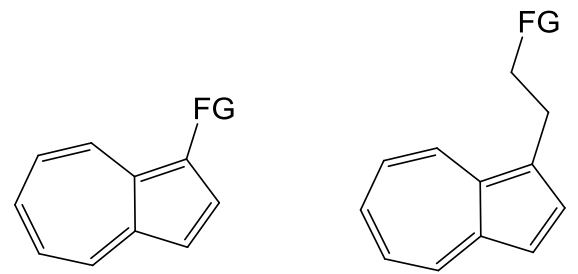

Figure 1.25 A generalised structure of an electronically connected (left) and an electronically isolated (right) protecting group

It was decided that the overall strategy for protecting group development was to be split into three generalised phases. These were:

1. The identification and synthesis of potential protecting groups.

2. Demonstration that the compound could, in principle be used as a protecting group (i.e. that a functional group can be added and cleaved in an acceptable yield with this compound).

3. Optimisation of conditions and exploration of orthogonal conditions.

Following these steps a total synthesis of some simple compound in order to demonstrate the protecting group's usefulness was planned.

\subsubsection{Previous examples of azulene-based protecting groups}

This research had its genesis in work done by M. Timmer et al. in $2007^{29}$ and led on to the creation of an azulene-based hydroxyl protecting group for carbohydrates. In this procedure, oxalyl chloride was added to azulene to form the azulene keto-acid chloride 11. This was subsequently added to a free hydroxyl-containing carbohydrate to form an azulene ketoester-protected carbohydrate of the general form 12 (Figure 1.26). 


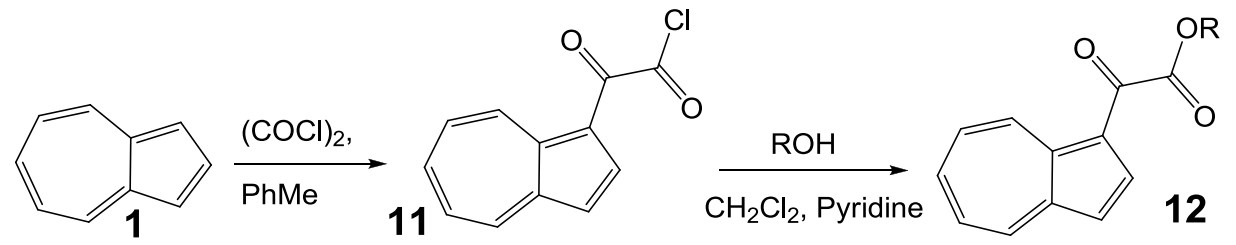

Figure 1.26 Reaction of azulene with oxalyl chloride followed by addition of the alcohol generates the ketoester-protected compound $\mathbf{1 2}$

A series of azulene-protected carbohydrates were synthesised and various protection and deprotection reactions were performed on other acid-labile groups on the carbohydrate structures in order to test the orthogonality of the azulene protecting group system. Following this, deprotection conditions were investigated. It was found that while the azulene keto ester could be cleaved efficiently using sodium methoxide in methanol, these conditions also tended to cleave other ester functionalities that were present. An alternate deprotection pathway was found that involved addition of $o$-diaminobenzene to the ketoester $\mathbf{1 2}$ in acetic acid and ethanol under reflux cleaving the ketoester in high yield and orthogonality to acetate groups to give the azulenic pyrazine 13 (Figure 1.27).

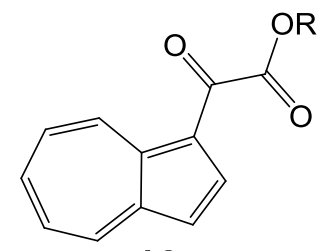

12

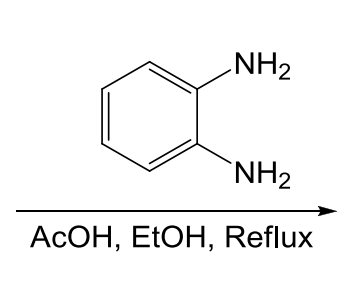

$\overrightarrow{\mathrm{AcOH}, \mathrm{EtOH}, \text { Reflux }}$

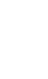

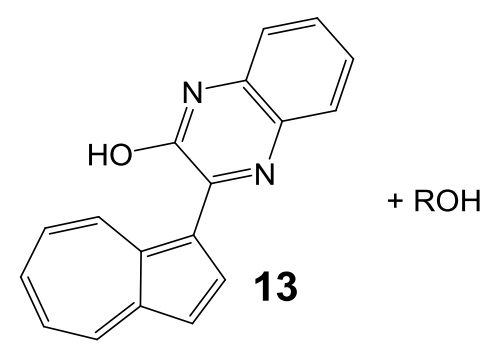

Figure 1.27 Addition of $o$-diaminobenzene to $\mathbf{1 2}$ in refluxing acetic acid cleaves the alcohol in high yield

The chromophoric protecting group reported by Aumüller et al. ${ }^{16}$ was designed for the protection of lone hydroxyl groups on carbohydrates. The carboxylic acid $\mathbf{1 4}$ was prepared from guaiazulene by deprotonation with LDA followed by addition of bromoacetic acid. 14 was then treated with carbonyldiimidazole in DMF prior to addition to the mannoside 16 (Figure 1.28).

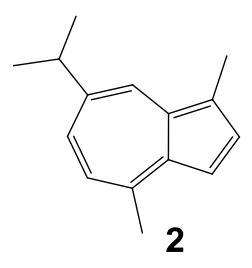

2

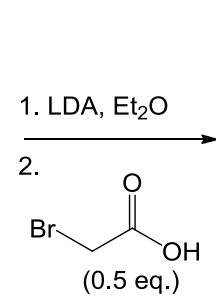

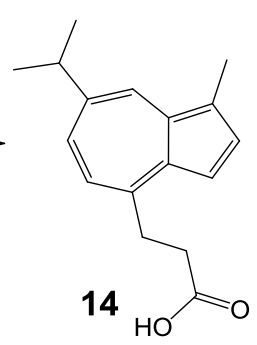
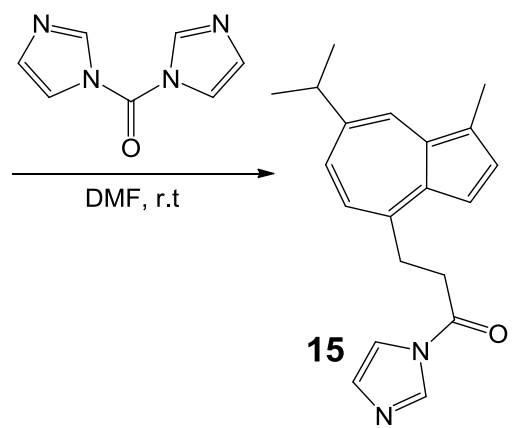

Figure 1.28 Preparation of the protecting group 14 followed by activation with carbonyldiimidazole 
The resultant guaiazulene-protected carbohydrate $\mathbf{1 7}$ was subsequently used in a variety of reactions without any observation of side reactions or deprotection. This guaiazulene protecting group can then be cleaved by using sodium methoxide in methanol (Figure 1.29 \& Figure 1.30).

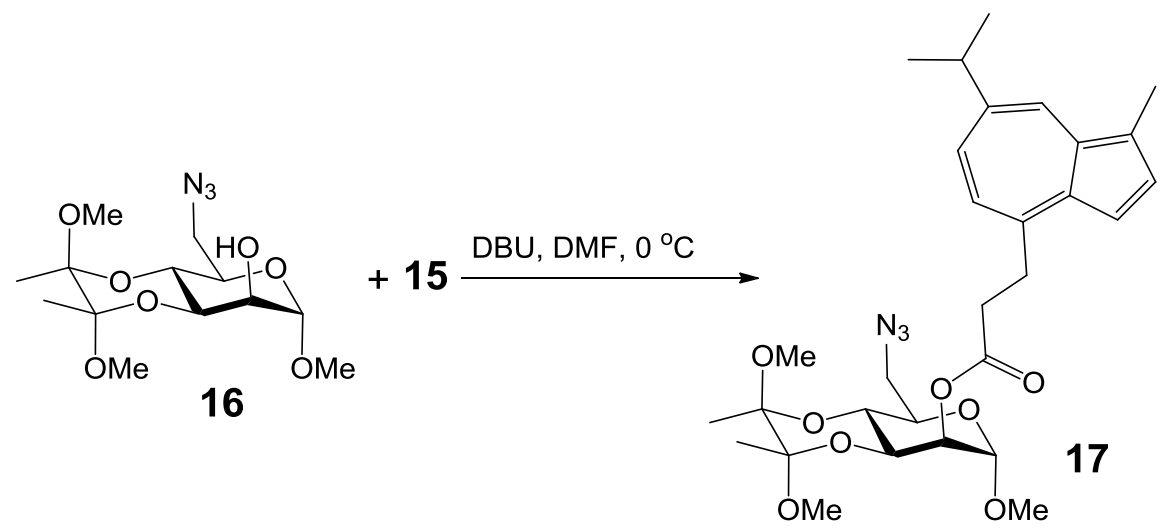

Figure 1.29 Coupling of the guaiazulene derivative $\mathbf{1 5}$ to the mannoside $\mathbf{1 6}$ gave the protected mannoside 17 in $46 \%$ yield

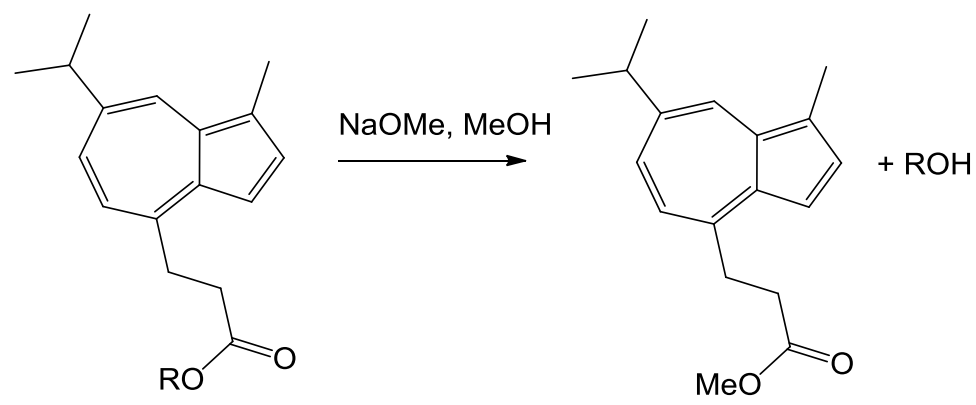

Figure 1.30 General cleavage conditions for the guaiazulene-based protecting group

An observation common to both papers is the ease of separation through column chromatography that these chromophoric protecting groups afford.

\subsection{Azulene functionalised polystyrene-divinylbenzene resins}

Over the past 40 years, organic polymer resins have seen a variety of uses across several sub-disciplines in chemistry. ${ }^{30}$ Much of their use has been in solid-phase peptide synthesis and combinatorial chemistry, but they can also be found as scavenger resins and as stationary phases in chromatography, along with several other niche uses. These resins are generally constructed of highly crosslinked organic polymers with varying functionality. ${ }^{30}$ One of the salient features of this crosslinking is the dynamic nature of the resin - although the polymer itself is solid, the interior of the resin can be described as a highly viscous liquid, as the flexible nature of the polymers allows a considerable degree of freedom within the resin. ${ }^{30}$ This behaviour 
can be altered markedly by the addition of a highly solvating solvent, allowing crosslinked polymers to swell to a considerable degree, as illustrated in Figure 1.31. This process can be considered analogous to the dissolution of linear polymers by a solvent.

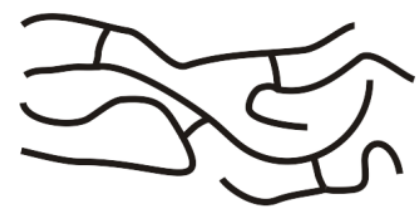

Non-swelled resin

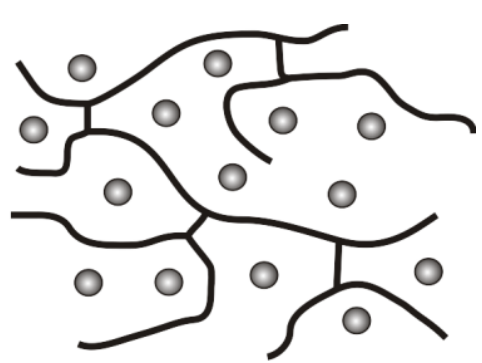

Swelled resin

Figure 1.31 Illustration of the interior polymeric structure of non-swelled and swelled resins

\subsubsection{Polystyrene-divinylbenzene (PS-DVB) resins}

The polystyrene-divinylbenzene resin is perhaps the most widely used polymer resin. $^{30}$ Synthesis is carried out by the radical polymerisation of the monomers styrene, divinylbenzene, and an appropriate functionalised styrene, as depicted in Figure 1.32. The degree of crosslinking is controlled by the quantity of divinylbenzene present, and usually ranges from $1 \%$ to $14 \%$.

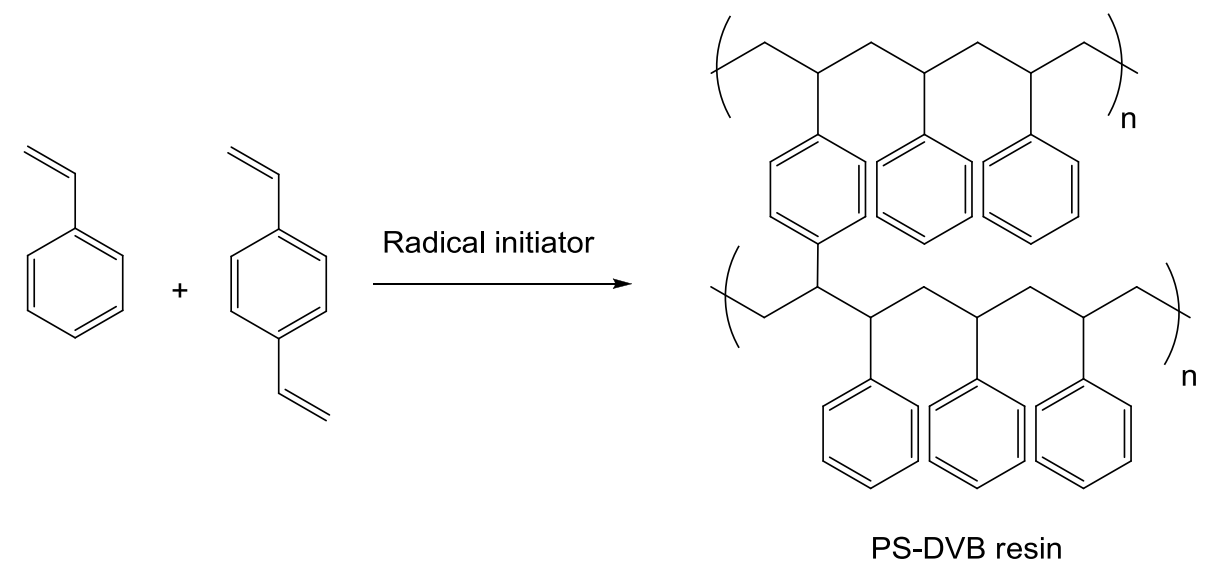

Figure 1.32 Formation of PS-DVB resin

High-swelling solvents for PS-DVB resins include dichloromethane, toluene and chloroform, while solvents such as water, DMSO and diethyl ether typically do not swell these resins appreciably. The extent of swelling necessarily depends on the degree of crosslinking within the resin, but in a solvent such as dichloromethane a $2 \%$ crosslinked resin can swell up to 8 times its original volume. ${ }^{31}$ The highly-permeable nature of these resins allows the functionalised groups throughout the resin to be accessible to both solvent and reagents, which in turn allows for complicated reactions and multi-step syntheses to take place entirely within the resin. 


\subsubsection{Use of PS-DVB resins in peptide synthesis and combinatorial chemistry}

One of the most widespread uses of PS-DVB resins is as a solid-phase support for peptide synthesis. The advantage of solid-phase synthesis is that purification is simple, with filtration of the resin from the reaction mixture often being the only necessary step. Each successive amino acid in a sequence is added onto a peptide covalently attached to the resin, followed by a filtration and deprotection cycle. This cycle has been optimised heavily and can now be successfully automated, allowing peptides of medium chain length (up to 100 amino acids long) to be quickly and efficiently synthesised.

The techniques developed for peptide synthesis have found much broader application in the field of combinatorial chemistry. Since one of the main focuses of combinatorial chemistry is the generation of large libraries of compounds, efficient synthesis and purification through solid-phase techniques is often necessary. This has led to the development of a much broader range of solid-phase methodology, such as the large variety of linkers available for different purposes, and the use of scavenger resins in purification.

\subsubsection{Use of PS-DVB as scavenger resins}

Scavenger resins serve primarily as a tool for the purification of reactions. Addition of the appropriate scavenger resin after a reaction is complete can neutralise remaining excess reagents, allowing simple filtration of the resin as a purification step. ${ }^{32}$

Since functionalised styrenes can easily be incorporated into PS-DVB resins, there is a wide range of different scavenger resins, each one designed to scavenge a certain reagent or group of reagents. Four examples of common scavenger resins are presented in Figure 1.33. 
<smiles>COc1ccc(CN)cc1</smiles>

Amine resin. Reacts with acids, acyl chlorides and other electrophiles<smiles>COc1ccc(C(=O)O)cc1</smiles>

Benzoic acid resin. Reacts with bases<smiles>Oc1ccc(CCN2CCCCC2)cc1</smiles>

Piperidine resin. Reacts with acids<smiles>O=Cc1ccc(OCCc2ccc(O)cc2)cc1</smiles>

Benzaldehyde resin. Reacts with nucleophiles

Figure 1.33 Examples of scavenger resins with different functions

A rather dramatic demonstration of the utility of scavenger resins can be seen in a paper by Ley et al. ${ }^{33}$ wherein a 3 step synthesis is performed using both resin-bound reagents and scavenger resins. A key feature of this paper was that purification was achieved at each step through use of the sulfonic acid scavenger resin and filtration alone. (Figure 1.34).

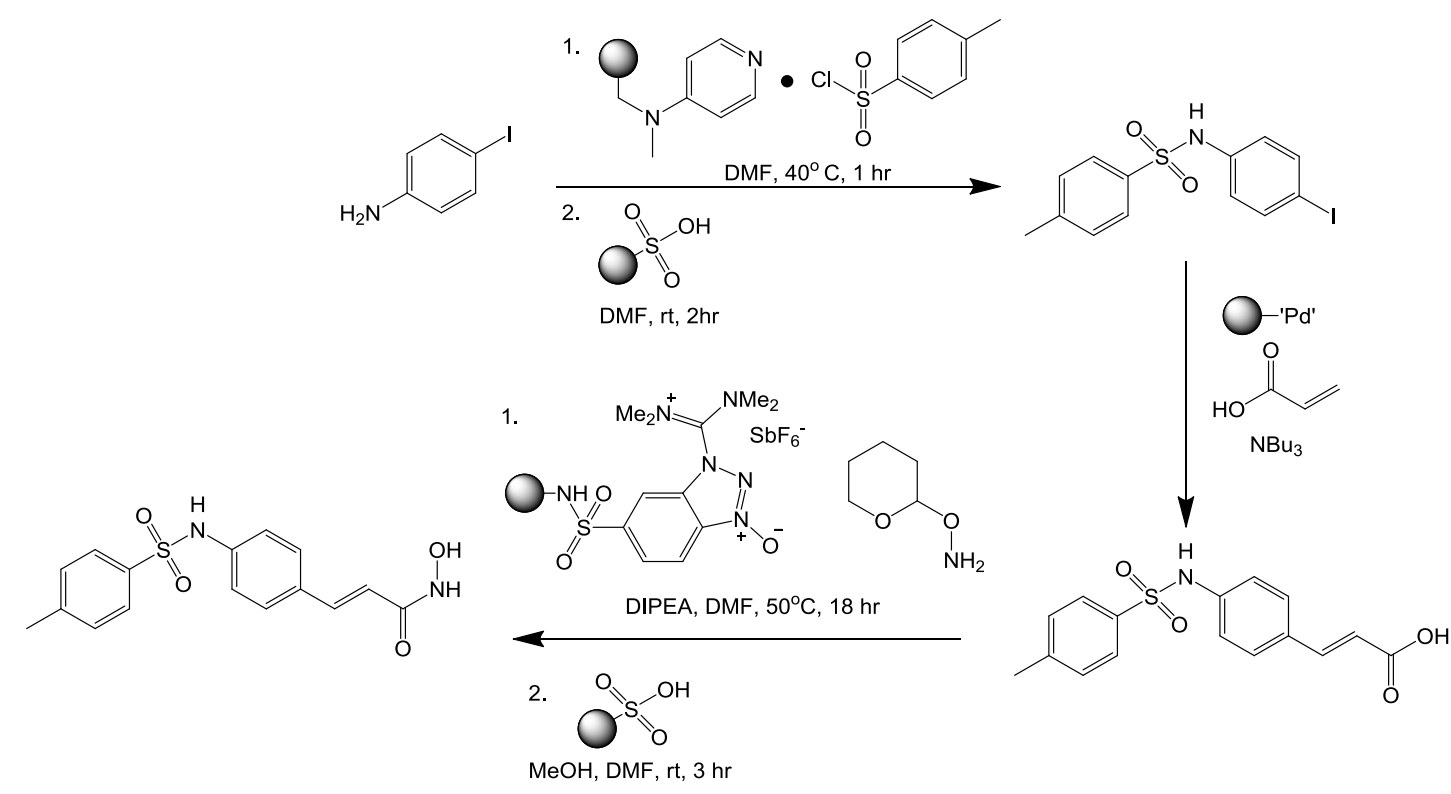

Figure 1.34 Example of synthesis performed using only solid-phase bound reagents and scavenger resins, with filtration being the only purification step

This process was successfully mechanised, allowing the product to be synthesised from $p$-iodoaniline automatically with no human intervention. Such procedures demonstrate the usefulness of scavenger resins, particularly within combinatorial chemistry but also within organic synthesis in general. 


\subsubsection{Motivation for the functionalisation of PS-DVB resins with azulene derivatives}

One of the primary aims of this research is to develop methodology for the functionalisation of PS-DVB resins with azulene derivatives for the purpose of creating self-indicating resins for various applications.

As a scavenger resin, azulene-functionalised PS-DVB resins would also be selfindicating, changing colour upon reaction with the relevant reagent. Since azulene itself is nucleophilic, such a resin could be used as a scavenger for electrophiles such as acyl chlorides, along with a predicted colour change from blue to red upon reaction. Further derivatives could conceivably be made that exhibit colour change in response to other reagents, such as acids, bases or nucleophiles. This allows one to continually add the appropriate resin into the reaction until no further colour change is observed, thereby removing the excess reagent. Furthermore, a reaction could be monitored by the selection of a resin that scavenges a reaction product. Providing one equivalent of the resin is added, once the colour change on the resin ceases the reaction can be considered complete. 


\section{Chapter 2}

\section{Results}

\subsection{Introduction}

At the beginning of this project, several syntheses were attempted without adequate knowledge of the chemistry of azulene derivatives, which led to early frustration. While azulene itself has been extensively investigated in the literature, knowledge of the derivatives we were interested in was lacking. Particularly in the case of deprotonation of the 4-methyl position of guaiazulene, there were only a handful of papers discussing this reaction, none of which gave any indications of its scope and limitations. As such, before the development of potential protecting group systems was pursued, it was deemed necessary to properly investigate the chemistry of the relevant azulene derivatives.

Initially, all the compounds synthesised in this research used azulene as a starting material. Although azulene itself is the simplest of the class of azulenes, it is moderately expensive and also laborious to synthesise. Guaiazulene, on the other hand, was much cheaper (up to 90 times cheaper based on 2008 Sigma-Aldrich prices) presumably due to it being a commonly-found natural product. Furthermore, the structure of guaiazulene offers more opportunities for derivatisation due to the presence of the alkyl groups. On this basis, we decided that the bulk of this research was to be carried out using the guaiazulene substrate.

\subsection{Reactions on the 3-position of guaiazulene}

The 3- position on the guaiazulene ring was one of the chosen sites of manipulation due to the nucleophilicity of this position and the subsequent wealth of electrophilic substitution reactions that could be carried out on this centre. 


\subsubsection{Vilsmeier-Haack formylation and related reactions}

The Vilsmeier-Haack formylation of guaiazulene was the first reaction that we studied in detail, as the aldehyde was deemed useful for further derivatisation. Vilsmeier reagent, a highly electrophilic chloroiminium species, can be generated by adding phosphorus oxychloride $\left(\mathrm{POCl}_{3}\right)$ to $\mathrm{DMF}$ at $0{ }^{\circ} \mathrm{C}$. This reagent is then added to the reaction mixture in order to generate an iminium species, which is then hydrolysed by $\mathrm{NaOH} / \mathrm{H}_{2} \mathrm{O}$ to generate an aldehyde (Figure $2.1 \&$ Figure 2.2).

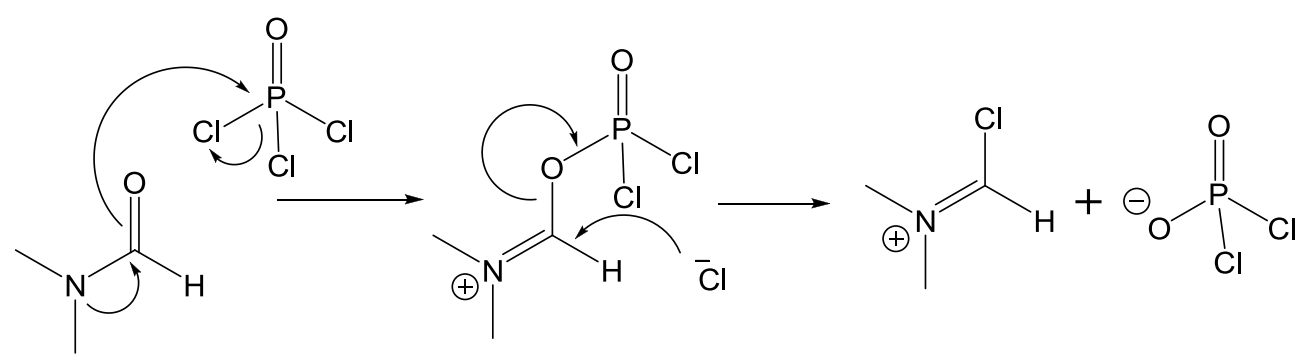

Figure 2.1 Generation of Vilsmeier reagent from $\mathrm{POCl}_{3}$ and $\mathrm{DMF}$
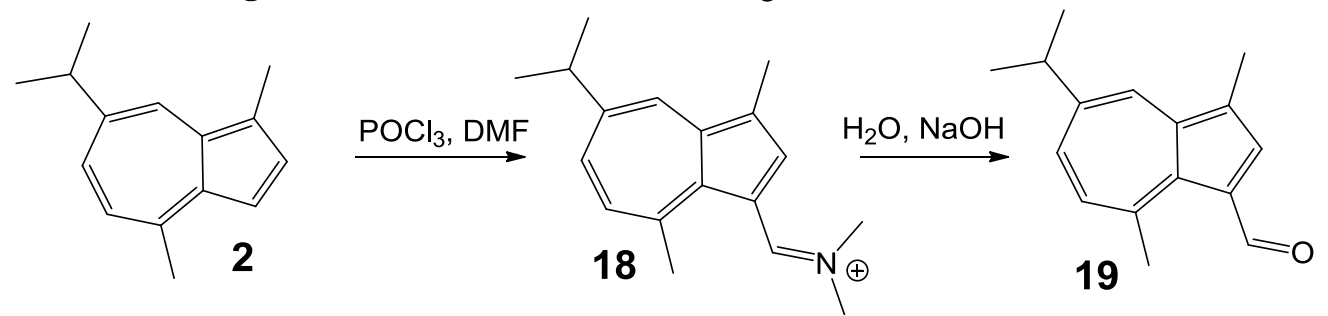

Figure 2.2 Reaction of $\mathbf{2}$ with Vilsmeier reagent followed by hydrolysis generates the aldehyde $\mathbf{1 8}$

The synthesis of the aldehyde $\mathbf{1 9}$ was seen as an important stepping stone to other reactions, owing to the number of ways a carbonyl can be manipulated. Initially, this reaction produced the desired purple aldehyde in $60 \%$ yield, but also afforded large quantities of a highly-polar deep red-orange compound. This compound was revealed by ${ }^{1} \mathrm{H}$ NMR to be the surprisingly stable iminium cation $\mathbf{1 8}$ through the identification of the singlet at 9.75 as the iminium proton and an additional singlet at 4.01 integrating for six protons belonging to the $N, N$-dimethyl moiety. Not only could this compound survive in open air for several days, it could also be purified by column chromatography on silica using a $\mathrm{MeOH} / \mathrm{CH}_{2} \mathrm{Cl}_{2}$ system as the eluent. The stability is due to the position of the iminium group on the azulene moiety allowing access to the tropylium-stabilised resonance structure through conjugation. Subsequent syntheses of the aldehyde 19 ensured complete conversion of 18 to 19 by hydrolysing for a longer period (>10 minutes) to ensure complete hydrolysis of the iminium moiety, improving the yield up to $77 \%$. 
The unusual stability of the iminium cation $\mathbf{1 8}$ opened up possibilities for nucleophilic substitution directly onto the iminium moiety and thereby would avoid the necessity of synthesising the aldehyde 19. This idea was explored by addition of cyclohexylamine to the iminium intermediate 18 (Figure 2.3). This resulted in the formation of a blue-green imine, which on addition to an acidic medium (such as a silica TLC plate) changed colour to the same orange-red colour as the iminium cation described earlier.

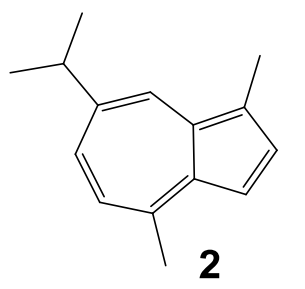

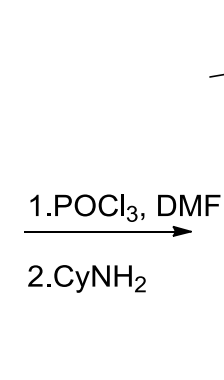

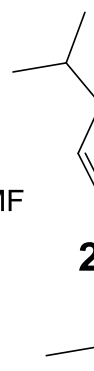

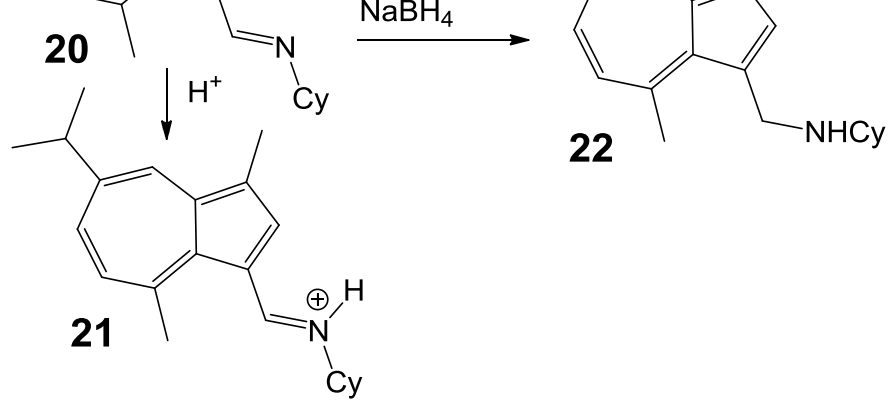

Figure 2.3 Reductive amination of the imine $\mathbf{2 0}$ to produce the amine $\mathbf{2 2}$

The imine $\mathbf{2 0}$ was reduced in situ to afford the amine $\mathbf{2 2}$ in 63\% yield, whose structure was confirmed through HMBC coupling between the cyclohexyl ring and the 3methylene on the guaiazulene moiety. This reaction was the first to be seriously considered as a potential protecting group system. The protection step would involve the addition of a primary amine to the iminium cation $\mathbf{1 8}$, followed by a reductive amination through addition of $\mathrm{NaBH}_{4}$, as depicted in Figure 2.4. Deprotection would be achieved through the addition of a suitable nucleophile under acidic conditions. We postulated that the ability of the guaiazulene moiety to stabilise a carbocation at the 3-position would facilitate both the $\mathrm{S}_{\mathrm{N}} 1$ mechanism (through the stable carbocation intermediate) and the $\mathrm{S}_{\mathrm{N}} 2$ mechanism (through stabilising conjugation of the $\delta^{+}$regions of the transition state by the azulene ring) for nucleophilic substitution at that position (Figure 2.5). Once protonated, the aminium moiety would act as a sufficiently good leaving group to be displaced by an appropriate nucleophile.
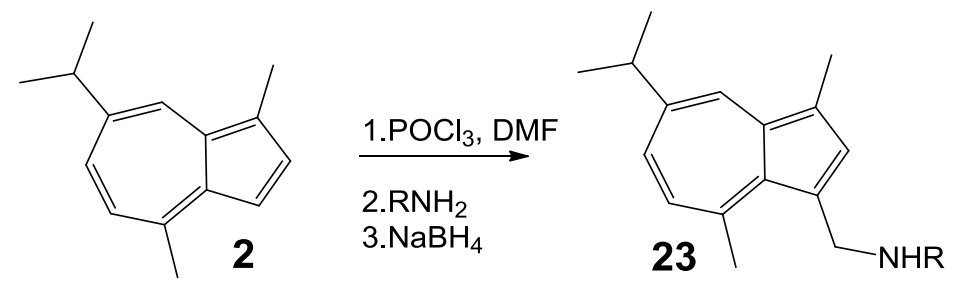

Figure 2.4 General procedure for the synthesis of guaiazulene-functionalised primary amines 


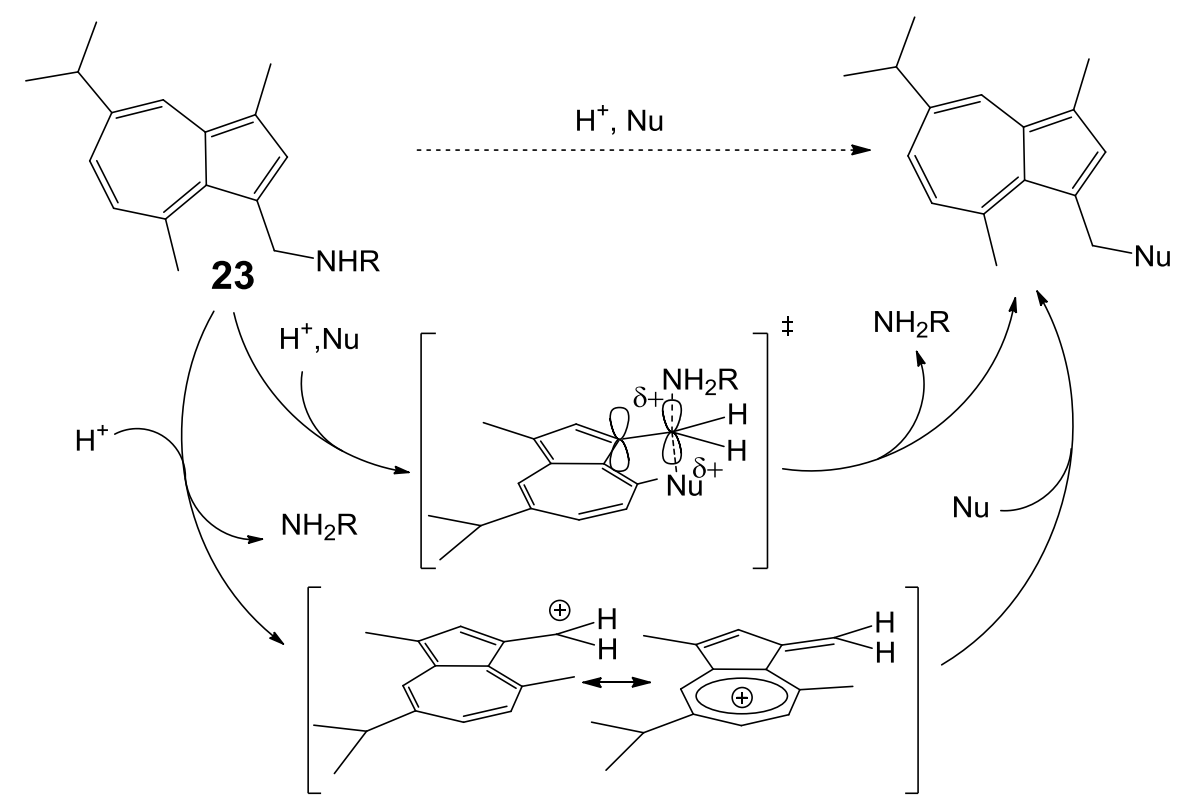

Figure 2.5 Proposed nucleophilic attack and deprotection of the amine 23. $\mathrm{S}_{\mathrm{N}} 2$ mechanism, top and $\mathrm{S}_{\mathrm{N}} 1$ mechanism, bottom.

Investigation of the protection and deprotection conditions turned up some problems with the system. First of all, in order to obtain complete conversion of the iminium $\mathbf{1 8}$ to the imine 20, a large excess of the amine was required. When 1 equivalent of the amine was added to the iminium, an equilibrium between the $\mathbf{1 8}$ and $\mathbf{2 0}$ was created, resulting after reduction in a 1:1 mixture of the amines $\mathbf{2 2}$ and $\mathbf{2 4}$.

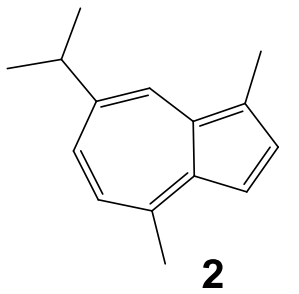

2

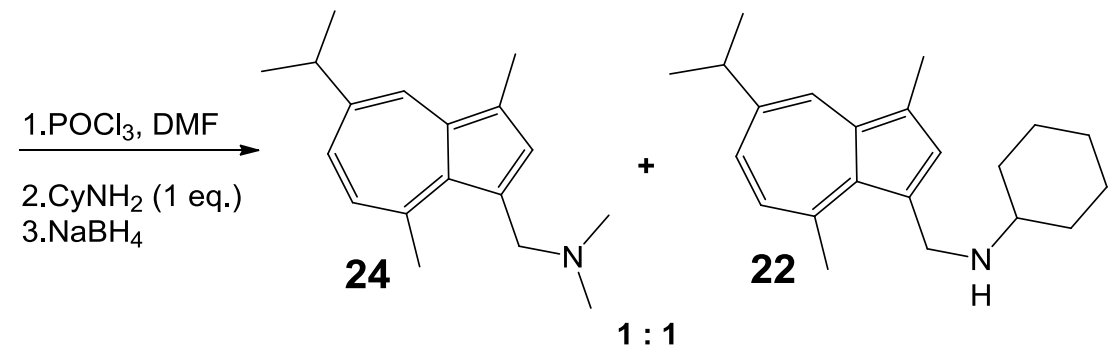

Figure 2.6 Use of one equivalent of cyclohexylamine gave rise to a 1:1 mixture of $\mathbf{2 4}$ and 22

The situation presented in Figure 2.6 is a problem for the application of this reagent as a protecting group, as the substrate in a protection reaction (in this case the amine) is by necessity the limiting reagent. In order to obtain complete conversion, a large excess of the iminium ion $\mathbf{1 8}$ would have to be added to the primary amine.

Secondly, and perhaps more critically, it was also found that the amine 22 was susceptible to degradation. Exposure to acidic media such as silica gel would result in the moderately fast degradation of $\mathbf{2 2}$, and exposure to air also resulted in degradation over several days. The principal degradation product was the short lived blue-green bis[7-isopropyl-1,4-dimethylazulenyl]methane 25, a compound which had appeared 
as a degradation product of similar 3-substituted azulene derivatives. The mechanism behind this conversion is suspected to involve the protonation of the amine $\mathbf{2 2}$, followed by ipso-substitution at the 3-position on another molecule of $\mathbf{2 2}$, resulting in the loss of 1 eq. of the primary amine and another 1 eq. of a formaldiminium ion.

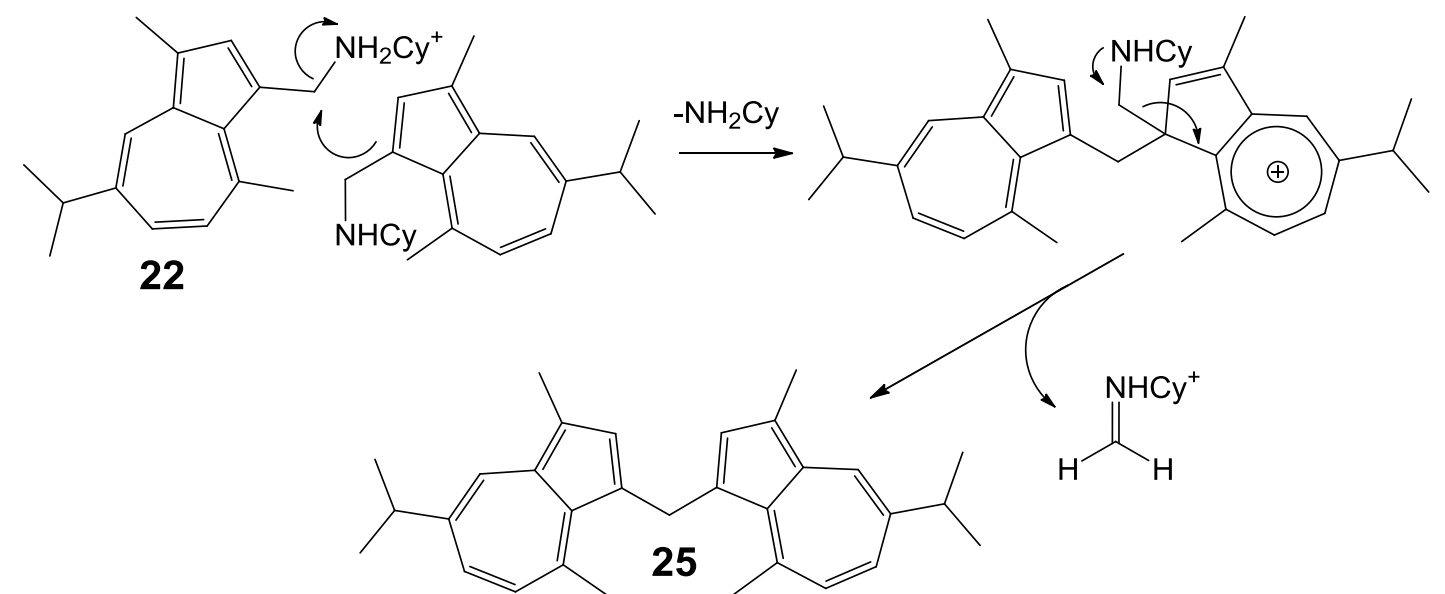

Figure 2.7 Proposed mechanism for the degradation of amine $\mathbf{2 2}$ into the bis(guaiazulenyl)methane $\mathbf{2 5}$.

This proposed mechanism is analogous to the mechanism proposed by Lash and coworkers of the synthesis of the calix[4]azulene $\mathbf{2 7}$ from the diol $26^{34}$

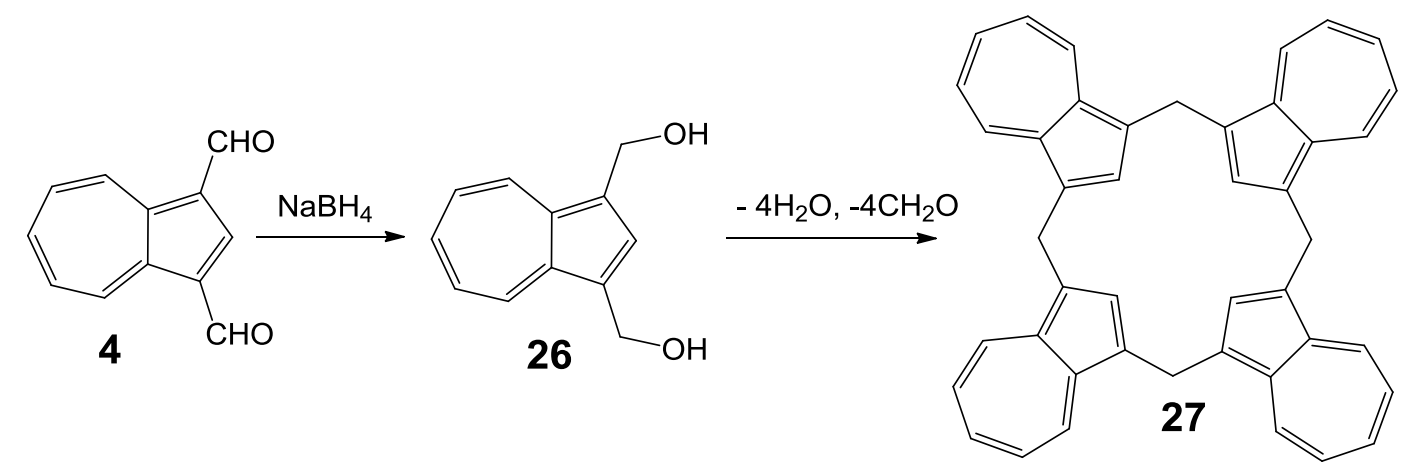

Figure 2.8 Reaction reported by Lash et al. in which ipso-substitution of $\mathbf{2 6}$ and loss of formaldehyde results in the formation of $\mathbf{2 7}$

This unwanted reactivity severely limits the usefulness of this system. There are possible ways in which this reactivity could be mitigated by reducing the nucleophilicity of the 3-position, but these are speculative at best. This chemistry still has the potential for use in a protecting group system after some modification, as ipsosubstitution may be shut down by addition of an electron withdrawing group to the azulene ring. The postulated mechanism of degradation suggests the amine is substituted by a nucleophile, which vindicates our original design. However, due to these ongoing difficulties with synthesis and degradation, this line of research was discontinued in favour of other research directions. 
The reductive behaviour of the aldehyde 19 was also investigated. A reduction was performed on 19 using $\mathrm{NaBH}_{4}$ in EtOH with the intention of producing the primary alcohol 28. It was intended that such an alcohol could be used in the protection of carboxylic acids as guaiazulene-functionalised esters. This alcohol was not observed (Figure 2.9). Instead, amongst the degradation products a very small amount of the 3methylguaiazulene 29 was present.

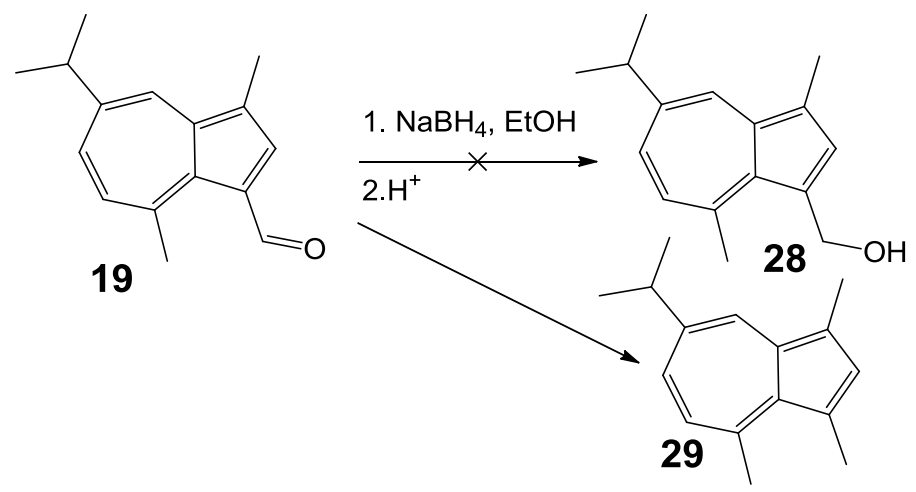

Figure 2.9 Reduction of the aldehyde 19 resulted in the formation of $\mathbf{2 9}$

We postulated that the formation of the weak Lewis acid $\mathrm{B}(\mathrm{OEt})_{3}$ in the reaction mixture was facilitating the complete reduction of the alcohol $\mathbf{2 8}$, by complexing to the hydroxyl group and facilitating nucleophilic attack by the sodium borohydride. To test the influence of the Lewis acid in this reduction, the reaction was repeated in the presence of the stronger Lewis acid boron trifluoride diethyletherate to successfully produce the 3-methyl guaiazulene $\mathbf{2 9}$ in $78 \%$ yield.

We considered this reaction to be valuable, as by blocking the 1- and 3-positions on the azulene ring, unwanted reactivity could be circumvented when manipulating other parts of the structure. However, this methyl derivative was observed to degrade completely over the course of a day. This proclivity towards degradation appears to be a common feature of all 3- alkyl derivatives of guaiazulene, and similar observations have been made of azulenes synthesised by Lash et $a . l^{35}$ and Razus et al. ${ }^{36}$

\subsubsection{Reactions with oxalyl chloride}

Oxalyl chloride is commonly used as a reagent for the introduction of acyl chlorides or esters to aromatic rings (Figure 2.10).

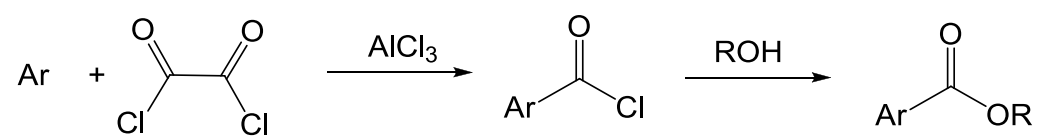

Figure 2.10 Formation of an aryl ester from an arene using oxalyl chloride and an alcohol 
When this reaction was attempted on guaiazulene, the product was not the expected ester, but rather the ketoester 30. Upon reaction with oxalyl chloride, an almost instantaneous colour change from blue to orange-red was observed. The alcohol was then added after approximately one minute, followed by pyridine to quench the reaction to obtain ketoester $\mathbf{3 0}$ (Figure 2.11).

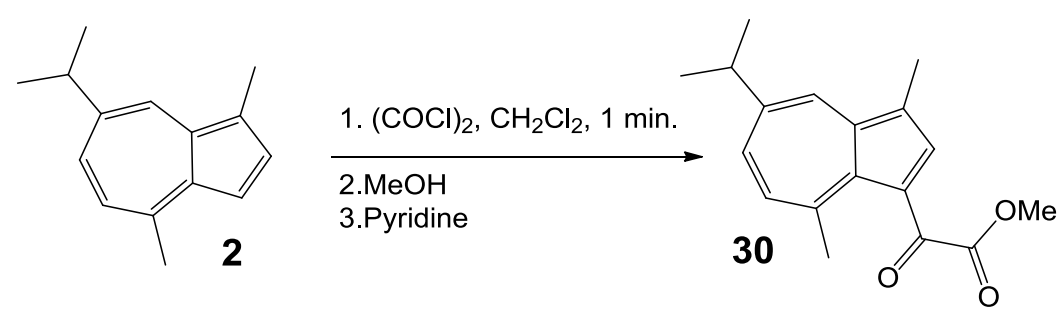

Figure 2.11 Formation of the ketoester 30 from 2 using oxalyl chloride

The original purpose of these reactions was to synthesise azulene esters. While the ketoester did find success as a protecting group ${ }^{29}$, it was the ester that we ultimately sought. A survey of the literature reveals that while oxalyl chloride is commonly used to generate an azulene ketoester, the ester itself is formed if the more reactive oxalyl bromide and a longer reaction period is used instead. ${ }^{37}$ Upon addition of oxalyl bromide to guaiazulene followed by addition of methanol after 20 minutes, the desired ester $\mathbf{3 1}$ was obtained as a viscous indigo oil in 52\% yield (Figure 2.12).
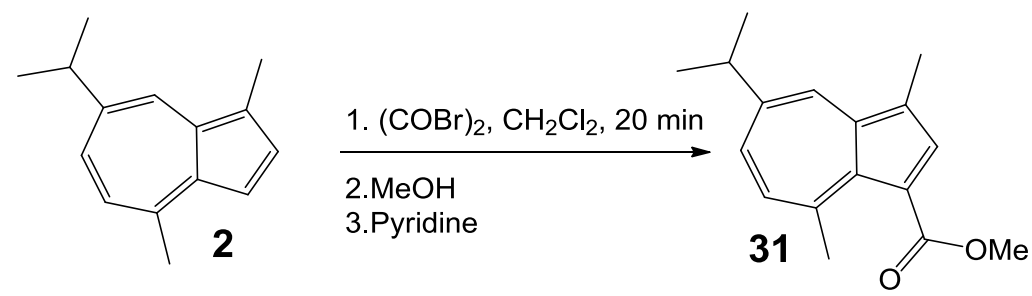

Figure 2.12 Formation of the ester $\mathbf{3 1}$ from 2 using oxalyl bromide

It was observed over the course of this reaction the colour would change from orangered to crimson. This colour change suggested by the rules outlined in section 1.2.3 that a change in the electron-withdrawing properties of the group at the 3-position had taken place, which in turn suggested that the more electron-withdrawing keto-acid bromide was being converted to the less electron-withdrawing acid bromide.

We proposed a radical mechanism for this reaction, which involves the loss of carbon monoxide in Figure 2.13.

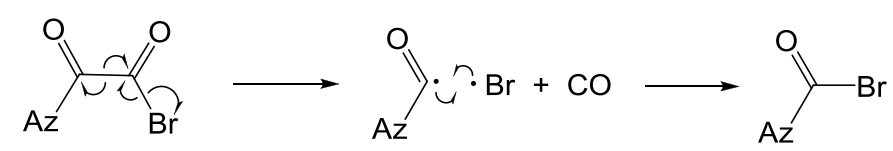

Figure 2.13 Proposed radical-based conversion mechanism from keto-acid chloride to acid chloride 
We subsequently hypothesised that this same process should occur, albeit more slowly, when oxalyl chloride is used as the electrophile. To test this, several reactions were undertaken in which oxalyl chloride was added to guaiazulene and the period before addition of alcohol was varied between 30 minutes and 20 hours. Periods of time under 1 hour would result in a mixture of the ketoester $\mathbf{3 0}$ and the ester $\mathbf{3 1}$, whereas longer periods in excess of 5 hours would produce a mixture of guaiazulene and unidentifiable degradation products, indicating a possible evolution of a second equivalent of carbon monoxide through a radical mechanism. Optimum yields of the ester 31 were obtained from a 1 hour period before addition of the alcohol, in one case giving $90 \%$ yield. This gave a powerful demonstration both of the usefulness of the rules that predict colour in azulene derivatives, and of the utility of oxalyl chloride for producing both ketoesters and esters depending on reaction time.

Amides were also synthesised using $(\mathrm{COCl})_{2}$ or $(\mathrm{COBr})_{2}$, albeit in lower yields than the corresponding esters. The guaiazulene $N, N$-diethylamide 32 was synthesised in $20 \%$ yield (Figure 2.14).

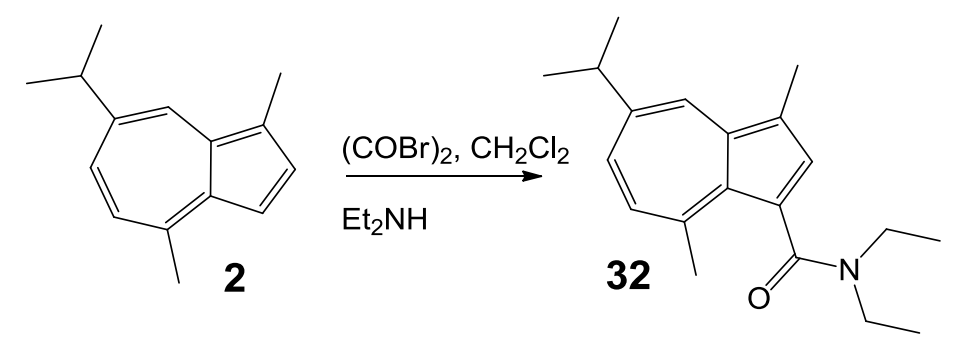

Figure 2.14 Synthesis of the amide 32 from 2

Following on from these results, the utility of guaiazulene acyl halides in FriedelCrafts acylation with other arenes was investigated. The ultimate goal of this line of investigation was to develop methodology to attach guaiazulene to a polystyrenedivinylbenzene resin, thereby achieving one of the aims of this research. Toluene was chosen as a suitable model for the PS-DVB resin as it resembles the phenyl rings in PS-DVB in terms of structure and reactivity.

Several Friedel-Crafts acylations were attempted on toluene using both guaiazulene acyl chloride and acyl bromide, generated in situ, with both aluminium trichloride and boron trifluoride diethyletherate used as Lewis acids. The general form of these reactions is depicted in Figure 2.15. 

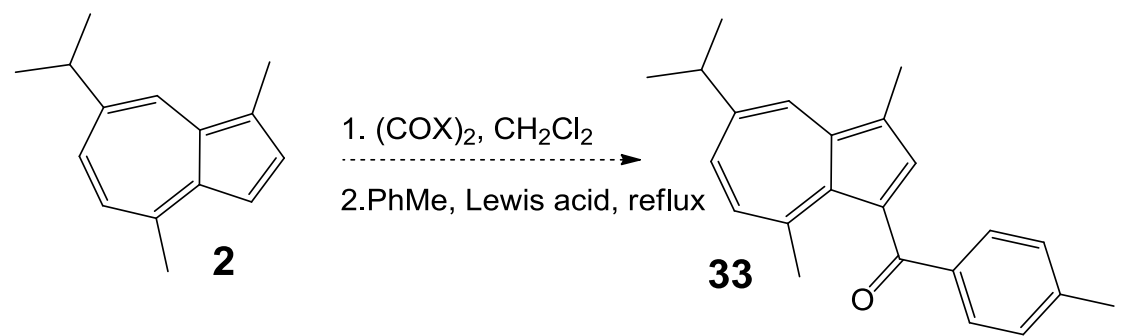

Figure 2.15 General form of the attempted syntheses of ketone $\mathbf{3 3}$ from $\mathbf{2}$

When the reaction was performed using oxalyl chloride and boron trifluoride diethyl etherate, a complex mixture of orange compounds were produced that degraded before they could be identified.

Upon replacement of oxalyl chloride with oxalyl bromide in the reaction, the formation of the unexpected guaiazulene ethyl ester $\mathbf{3 4}$ was observed in 27\% yield (Figure 2.16). The only possible known source of the ethoxy group is from the boron trifluoride diethyl etherate, but the mechanism of the formation of this compound is unknown.

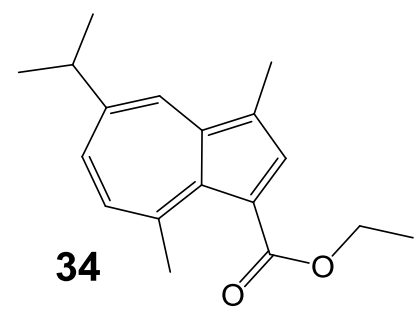

Figure 2.16 The guaiazulene ethyl ester 34

When aluminium trichloride was used as the Lewis acid, a crimson-red compound was formed. This compound was difficult to isolate, as it is highly polar and degraded rapidly in the presence of water.

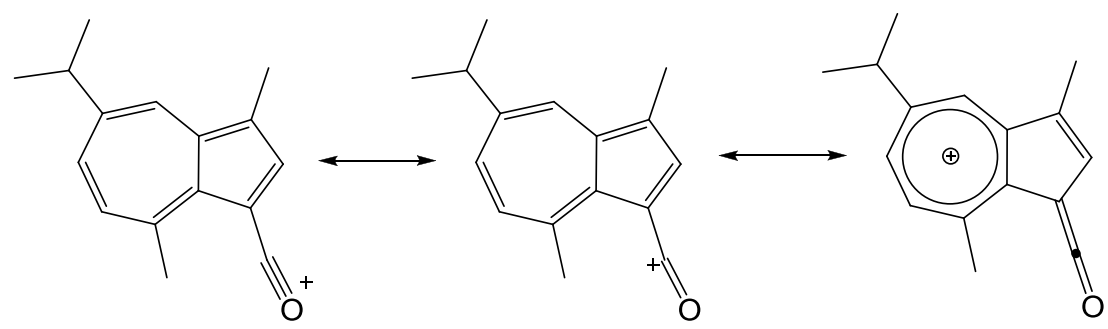

Figure 2.17 Postulated identity of the crimson-red compound

It was postulated that this might be an acylium cation intermediate (Figure 2.17), as there is precedent for their existence ${ }^{38}$ so a vibrational frequency DFT calculation on this compound was performed using the EDF2 potential (designed specifically for accurate calculation of vibrational frequencies) with the $6-31+\mathrm{G}^{*}$ basis set. The salient $\mathrm{C}=\mathrm{O}$ stretch was calculated at $2230 \mathrm{~cm}^{-1}$, which was completely absent in the 
IR spectrum. This result refutes the postulated acylium structure. Unfortunately, neither a meaningful ${ }^{1} \mathrm{H}$ NMR nor a ${ }^{13} \mathrm{C}$ spectrum could be produced, so this compound could not be characterised further. Due to the difficulty encountered in this line of research, other methods for introducing guaiazulene to a PS-DVB resin were sought.

\subsubsection{Summary of section 2.2}

The chemistry of the guaiazulene aldehyde 19 was investigated, leading to the discovery of the total reduction of the aldehyde to the methylguaiazulene $\mathbf{2 9}$ and to the investigation of reductive amination and nucleophilic cleavage of an amine as a protecting group strategy. While the chemistry itself was sound, the amine 22 was unsuitable as a protecting group as it reacted with itself under mild acidic conditions.

The ester 31 and amide $\mathbf{3 2}$ were synthesised. A convenient route to the synthesis of guaiazulene acyl chlorides was hypothesised and subsequently established, circumventing the need for the use of the more expensive oxalyl bromide. Use of the acyl chloride and acyl bromide in Friedel-Crafts acylation with toluene was investigated, with negative results. This precludes the use of acylation with a guaiazulene acyl halide onto PS-DVB resins. 


\subsection{Manipulation of the 4-methyl group}

\subsubsection{Introduction}

Aumüller and co-workers demonstrated that a proton on the 4-methyl position can be removed with LDA, generating the relatively stable carbanion 35 (Figure 2.18). This guaiazulenide anion is stabilised by the presence of the cyclopentadienide resonance form, and it is thought the pKa of this proton lies somewhere between 20-30.

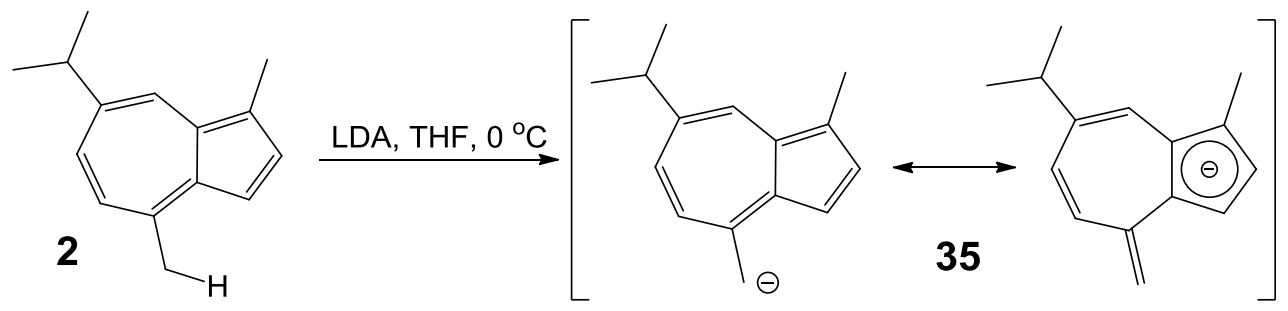

Figure 2.18 Deprotonation of guaiazulene at the 4-methyl position affords the guaiazulenide anion $\mathbf{3 5}$

The carbanion produced is much more nucleophilic than the 3- position of guaiazulene. Although enhanced nucleophilicity of the 5-membered ring may be predicted through resonance, only nucleophilic attack from the 4-methyl position was ever observed during this project. We found this reaction to be highly versatile, as the carbanion is sufficiently nucleophilic to react with alkyl halides and most carbonyl groups, giving access to a wide range of possible products and opening up new areas of chemistry to be exploited for protecting group development.

\subsubsection{Deprotonation of guaiazulene}

This deprotonation was first explored using chemistry previously reported by Aumüller and co-workers. ${ }^{16}$ In this paper, a procedure was described in which a solution of LDA was added to guaiazulene followed by addition of paraformaldehyde to afford the compound 36, as depicted in Figure 2.19.
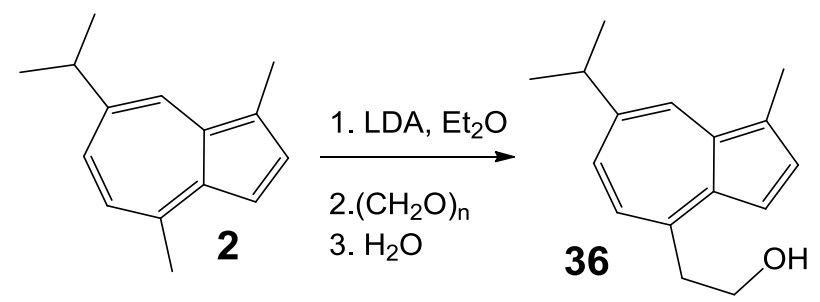

Figure 2.19 Nucleophilic attack by $\mathbf{3 5}$ on formaldehyde affords the alcohol $\mathbf{3 6}$

A colour change upon deprotonation is expected, as the electronic structure is altered by the presence of the exocyclic methylene and the negative charge. 
Unfortunately, no colour change observations were reported in this paper, meaning there was no prior way of assessing if the deprotonation had gone to completion.

Initial attempts were made to repeat this reaction using various bases such as sodium amide and $n$-butyllithium, as they were freely available and did not have to be prepared. Use of these bases either resulted in no reaction or complete degradation upon addition to guaiazulene. This reaction was successfully repeated when LDA generated from diisopropylamine and $n$-butyllithium in THF - was added to a solution containing guaiazulene. This resulted in a colour change to orange-brown, a colour which was in rough agreement with TDDFT UV-Vis calculations on the guaiazulenide anion species. To this guaiazulenide anion a large excess of paraformaldehyde was added and the orange-brown reaction mixture changed colour back to blue in a further few seconds. The compound 36 was obtained in $22 \%$ yield and the NMR spectral data matched those of the compound reported by Aumüller et al.

Further attempts were made to improve reaction conditions, and an optimised yield of $67 \%$ was obtained after the method of addition of paraformaldehyde was altered. Instead of addition of paraformaldehyde as a powder, thermal cracking followed by direct cannulation of the evolved formaldehyde gas into the reaction mixture resulted in these higher yields.

The scope of this deprotonation reaction was explored further. To test whether alkyl halides would also react, benzyl chloride was added to a solution containing the guaiazulenide anion (Figure 2.20).

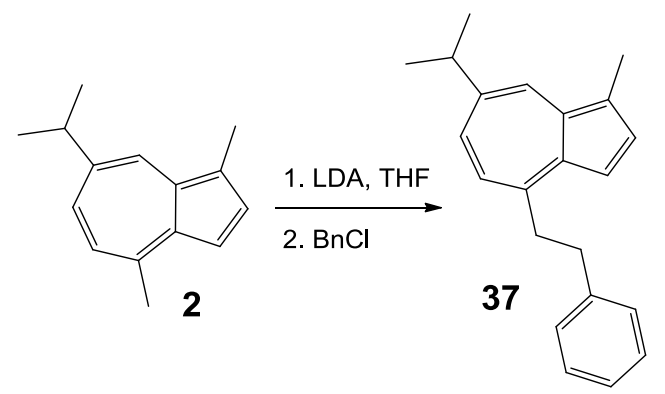

Figure 2.20 Reaction of $\mathbf{3 5}$ with benzyl chloride produces $\mathbf{3 7}$

The benzyl chloride also reacts with the guaiazulenide anion, albeit more slowly. Based on the observed colour change from orange to blue, the reaction time is of the order of 1 minute. The compound $\mathbf{3 7}$ was obtained in a $48 \%$ yield and the structure 
confirmed through HMBC coupling between the guaiazulene and phenyl group through the ethylene link.

Reaction of the guaiazulenide anion with 1,2-dibromoethane was also investigated (Figure 2.21). 3.5 equivalents of 1,2-dibromoethane was added to the guaiazulenide anion and the monosubstituted product was obtained in $21 \%$ yield. Interestingly, no disubstituted product was observed. This reaction also demonstrates that the presence of the benzyl group is not necessary to allow nucleophilic substitution on the alkyl halide.

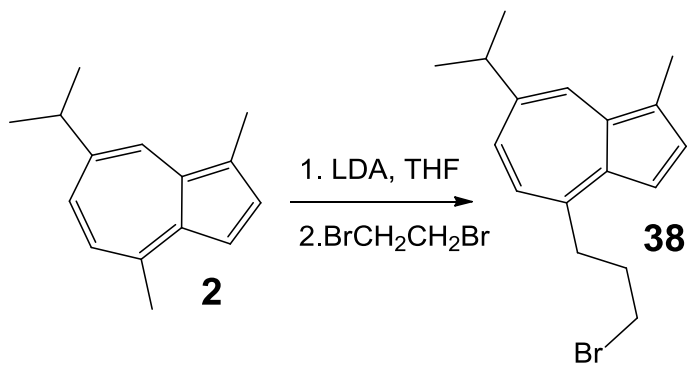

Figure 2.21 The guaiazulenide anion 34 reacts with 1,2-dibromoethane to produce 38

As part of a side project, the bromoazulene $\mathbf{3 8}$ was converted into the thiol $\mathbf{3 9}$ by addition of thiourea in refluxing EtOH overnight followed by addition of aqueous $\mathrm{NaOH}$, resulting in a $68 \%$ yield (Figure 2.22). The presence of the carbon-sulfur bond was confirmed by the diagnostic ${ }^{13} \mathrm{C}-{ }^{1} \mathrm{H}$ coupling of $140 \mathrm{~Hz}$ on the attached carbon.

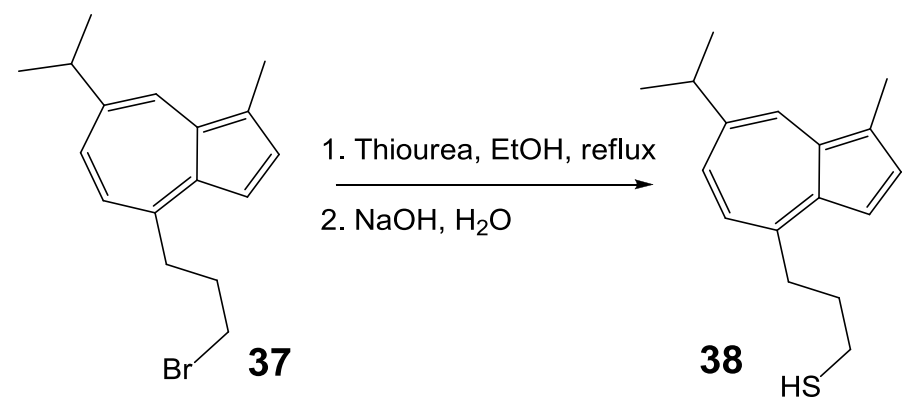

Figure 2.22The bromide 38 was converted to the thiol 39

The purpose of this thiol was to create a chromophore that could be easily bound to a metal nanoparticle. This is part of ongoing research in Surface Enhanced Raman Spectroscopy (SERS) wherein the dependence of distance between the chromophore and the metal nanoparticle on the quenching of fluorescence is investigated. 
These reactions demonstrate the synthetic versatility of deprotonation at the 4-methyl position. Owing to the wide range of available organic halides, use of the guaiazulenide anion provides a route to many potentially useful derivatives.

Both Vilsmeier-Haack formylation and acylation with oxalyl chloride were performed on compound 37 (Figure 2.23). This was partly to assess whether the presence of the phenethyl group on the 4-position would result in any unusual reactivity, but also as a model for on-resin chemistry described in section 2.4.4.

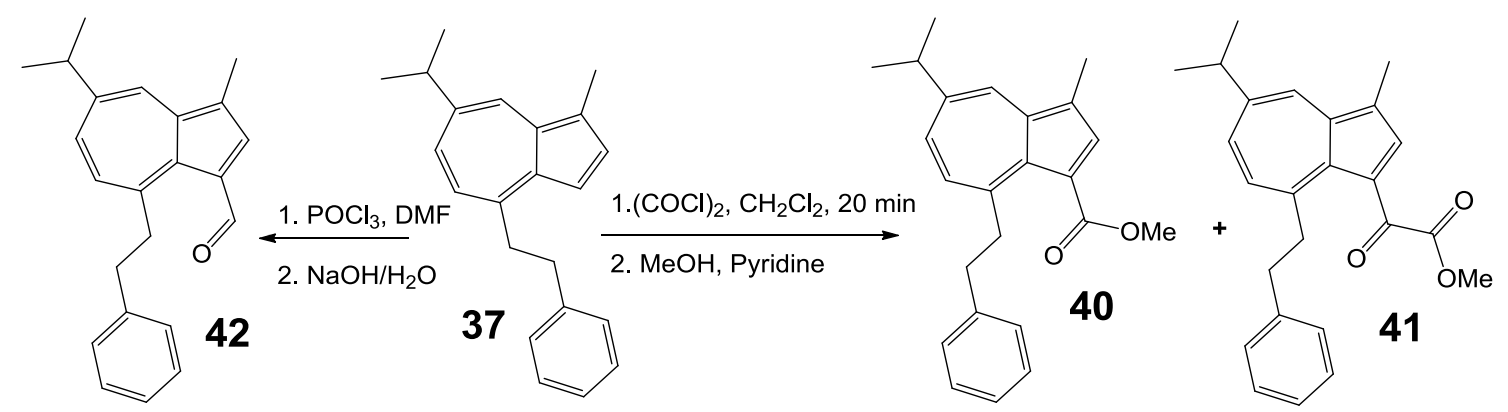

Figure 2.23 The derivatives 40, 41, 42 were synthesised from 37

The aldehyde $\mathbf{4 2}$ was obtained in 36\% yield, and the ester $\mathbf{4 0}$ and ketoester $\mathbf{4 1}$ were obtained in $29 \%$ and $47 \%$ yields respectively. A reaction time of 20 minutes between addition of $(\mathrm{COCl})_{2}$ and $\mathrm{MeOH}$ was deliberately chosen so that a mixture of both products would be obtained. Behaviour of $\mathbf{3 7}$ towards these conditions was very much similar to that of guaiazulene.

\subsubsection{Deprotonation of guaiazulene derivatives}

The next step in this research was to explore the reactivity of various guaiazulene derivatives towards deprotonation of the 4-methyl group. In particular, it was expected that guaiazulenes substituted with electron withdrawing groups would deprotonate more readily than guaiazulene itself. Unlike guaiazulene however, these derivatives have the potential to react with themselves intermolecularly, and could conceivably form dimers or (more likely) oligomers, as illustrated in Figure 2.24. It was therefore deemed necessary that before any reactions involving electrophilic reagents are undertaken, the reactivity of the deprotonated derivative with itself be properly described. 


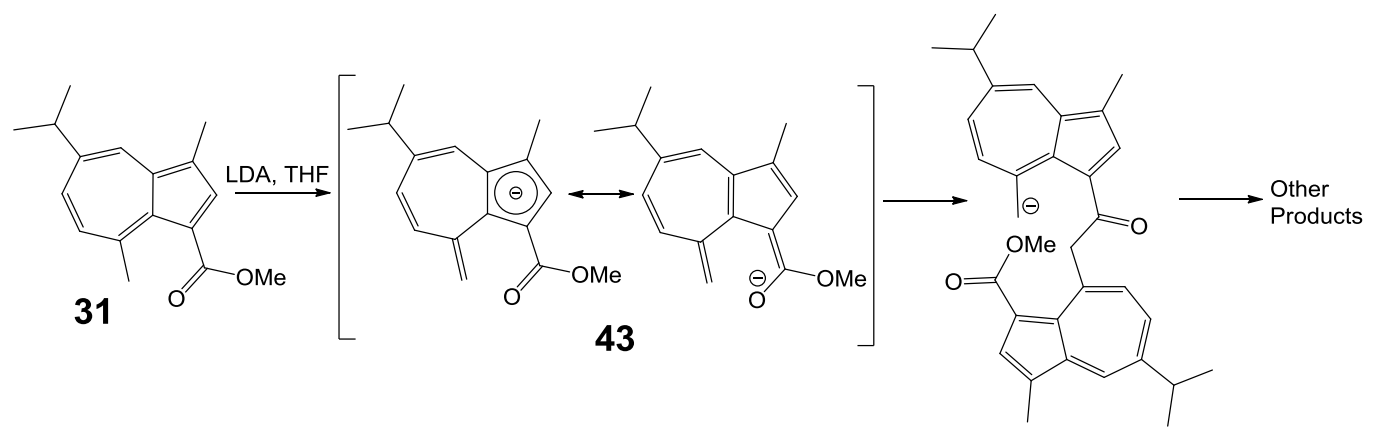

Figure 2.24 Postulated degradation pathway of the guaiazulenide ester 43

Deprotonation reactions on the guaiazulene methyl ester $\mathbf{3 1}$ were carried out.

2.3 equivalents of LDA were added dropwise to a solution of $\mathbf{3 1}$ in THF. An instantaneous colour change from purple to yellow was observed, indicating the methyl ester is deprotonated much more readily than guaiazulene, as we expected. After approximately a minute, the yellow colour had changed to gray. This mixture was left for a further 1 hour before being reprotonated by the addition of $\mathrm{H}_{2} \mathrm{O}$. After this period, some compounds were present by TLC that were unidentifiable by NMR, including a highly polar grey-purple substance with a tendency to streak, whose behaviour and colour suggested oligomerised starting material. This result indicates that the ester $\mathbf{3 1}$ is not stable upon deprotonation and reacts with itself over the course of minutes.

The competition between this oligomerisation and reaction with a suitable electrophile was investigated. Paraformaldehyde was chosen for this role as it was the fastestreacting electrophile that had been used at the time of this investigation. Immediately after deprotonation of $\mathbf{3 1}$, an excess of paraformaldehyde powder was added to the reaction mixture and a colour change from yellow to purple was observed. The majority of the products were unidentifiable by NMR, and as before, a baselining purple-grey material was present. Starting material was also observed, along with a small amount (18\% yield) of a magenta compound. This structure lacked a shift for the methoxy group in the ${ }^{1} \mathrm{H}$ NMR, suggesting a transesterification product. The structure was confirmed by MS to be the 7- membered lactone 44. The expected product, $\mathbf{4 5}$ was not observed, however (Figure 2.25). 

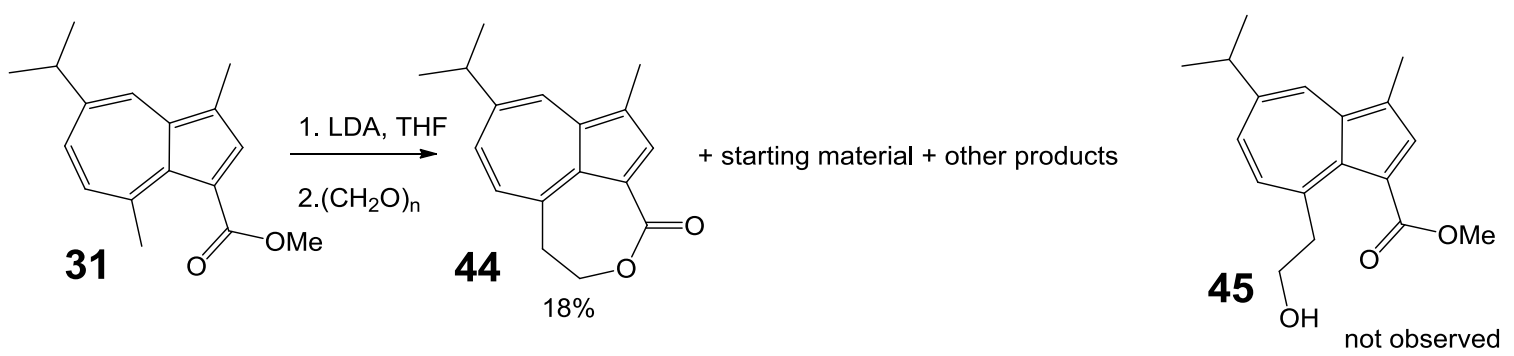

Figure 2.25 The lactone $\mathbf{4 4}$ was the only identifiable product from the deprotonation and subsequent treatment of $\mathbf{3 1}$ with paraformaldehyde

The deprotonation of $\mathbf{3 1}$ was repeated again at $-78{ }^{\circ} \mathrm{C}$, followed by addition of a vast excess (163 equivalents) of benzyl chloride. Since it had already been established that a more reactive electrophile - paraformaldehyde - was barely able to compete with the oligomerisation of the guaiazulenide ester, it was expected that benzyl chloride would not compete at all at $0{ }^{\circ} \mathrm{C}$. By cooling down the reaction vessel to $-78{ }^{\circ} \mathrm{C}$ and adding an excess of benzyl chloride, we thought that this might drive the substitution reaction while hindering oligomerisation. If the kinetic barrier to oligomerisation was higher than nucleophilic attack on the benzyl chloride, substitution would be observed over oligomerisation. At $-78{ }^{\circ} \mathrm{C}$, the deprotonation proceeds much more slowly, and after addition of benzyl chloride the reaction mixture changed colour from orange to purple over the course of half an hour. Products obtained were starting material (37\%) and 46 (29\%), whose presence can be attributed to the small quantities of benzaldehyde impurity in the large quantity of benzyl chloride used. The expected product was not observed.

Following these results, it was decided that the increased acidity of the 4-methyl position was not worth the difficulties associated with oligomerisation in these reactions.

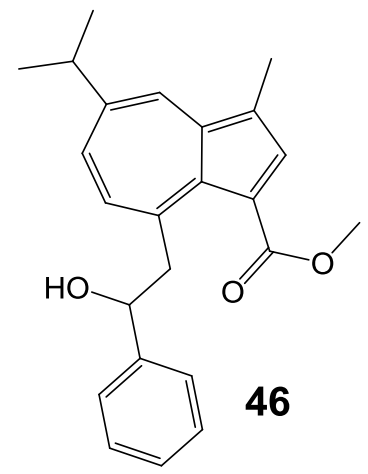

Figure 2.26 The formation of $\mathbf{4 6}$ was attributed to benzaldehyde impurity in the benzyl chloride reagent 
The tert-butyl ester of guaiazulene 47 was synthesised through addition of tertbutanol to the guaiazulene acyl chloride as described previously. This compound was also subjected to LDA deprotonation and similar reactions in order to assess the effect of the bulky tert-butyl group on oligomerisation and reactivity in general.

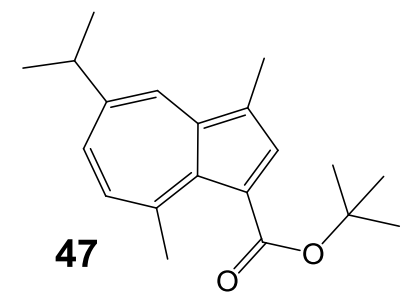

Figure 2.27 The ester 47 was synthesised from guaiazulene using oxalyl chloride and tert-butanol

As before, the behaviour of the guaiazulenide tert-butyl ester towards oligomerisation was investigated. LDA was added to the tert-butyl ester in two separate experiments at $0{ }^{\circ} \mathrm{C}$. In the first experiment, the reaction was quenched with $\mathrm{H}_{2} \mathrm{O}$ after 30 seconds. Almost all starting material was recovered, with no reaction products observed. In the second experiment, a 15 minute time period before quenching was used. Again, starting material was recovered in $95 \%$ yield, with only traces of unidentified material present. During the reaction period, no gradual colour change from yellow was observed, unlike the case with the methyl ester. This demonstrates that the tert-butyl group is sufficiently bulky to hinder the nucleophilic attack at the carbonyl group.

We thought that the enhanced reactivity of the ester $\mathbf{4 7}$ towards LDA and the stability afforded by the tert-butyl group would make it a viable alternative to guaiazulene in the synthesis of several derivatives. Several reactions common to this project were then repeated on 47. The results are summarised in Figure 2.28.

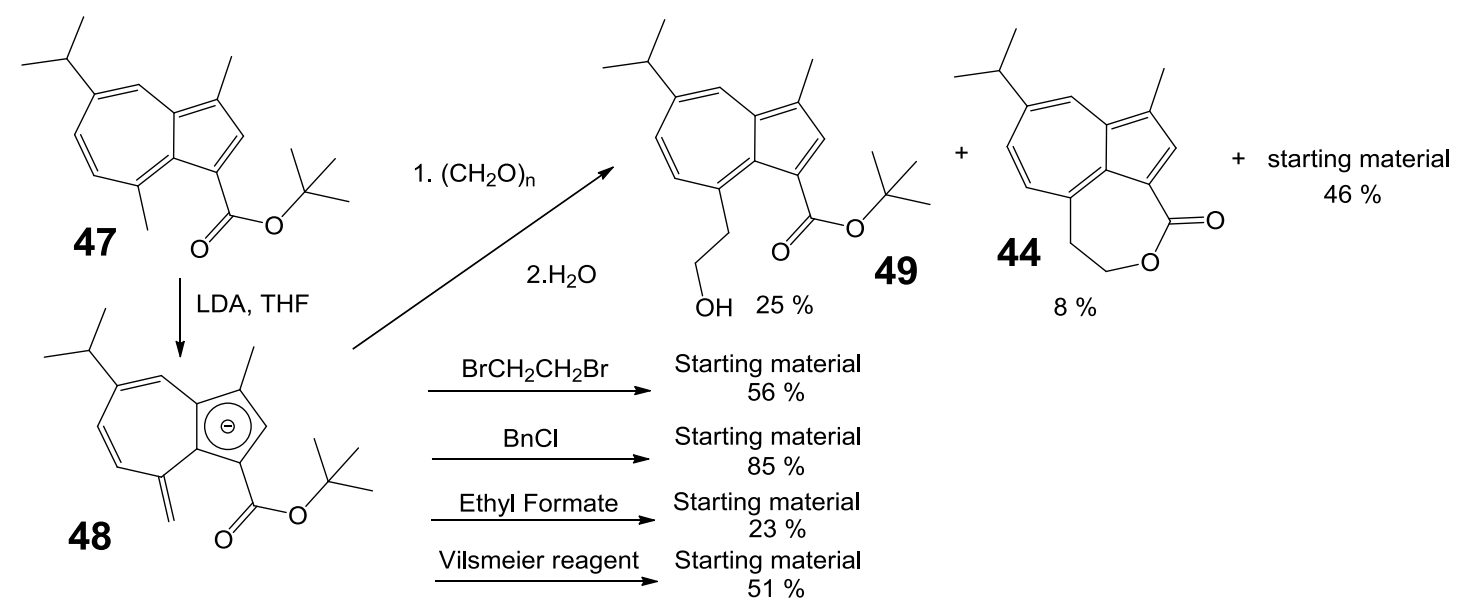

Figure 2.28 Deprotonation of $\mathbf{4 6}$ to form the anion $\mathbf{4 7}$, which was reacted with several different electrophiles 
Addition of paraformaldehyde resulted in a $25 \%$ yield of the intended product 49 plus $8 \%$ yield of the 7 -membered lactone 44 . Nearly $50 \%$ starting material was recovered. Out of all the subsequent reactions, involving 1,2-dibromoethane, benzyl chloride, ethyl formate and the chloroiminium Vilsmeier reagent, only starting material and other unidentifiable oligomeric products were produced. The colour of the deprotonated species would normally only change gradually from yellow to purple, possibly due to imperfections in the septa used allowing for slow diffusion of air into the flask.

There are two possible explanations for this lack of reactivity towards electrophiles. One is that the tert-butyl group provides too much steric hindrance for any large electrophiles to approach the carbanion, while allowing water or $\mathrm{H}^{+}$to reprotonate it. In this sense, the carbanion is acting as a non-nucleophilic base. This theory is consistent with the observation that only the relatively small formaldehyde reacted with the deprotonated species. The other explanation is that the resultant carbanion with the negative charge stabilised through resonance by both the 5-membered ring and the ester - has been rendered too unreactive thermodynamically to attack most electrophiles. Both of these effects are likely to contribute to some extent to the lack of reactivity observed.

\subsubsection{Summary of section 2.3}

Reaction of the guaiazulenide anion with various electrophiles afforded compounds such as the alcohol 36, the 2-ethylphenyl azulene derivative $\mathbf{3 7}$, and the bromide $\mathbf{3 8}$, thus demonstrating the potential for the synthesis of a large number of derivatives. The compound $\mathbf{3 7}$ was subsequently reacted with Vilsmeier reagent and with oxalyl chloride to afford the derivatives $\mathbf{4 2}, \mathbf{4 0}$, and $\mathbf{4 1}$. The bromide 38 was converted into the thiol 39, for use as a chromophore in the investigation of fluorescence quenching in SERS.

The deprotonation of the guaiazulene methyl ester $\mathbf{3 1}$ and the tert-butyl ester $\mathbf{4 7}$ were investigated. The methyl ester reacted rapidly with itself upon deprotonation, whereas the tert-butyl ester was much more stable in this regard. The only reaction observed to proceed on these two compounds is that with paraformaldehyde, which severely limits the usefulness of these esters in the synthesis of derivatives. 


\subsection{Coupling with polystyrene-divinylbenzene resins}

\subsubsection{Introduction}

It was one of the major goals of this research to couple an azulene moiety to a PSDVB resin. We envisaged that such a resin might find a variety of uses, with use as a scavenger resin for various reagents being a relatively easily tested example.

Originally, it was planned that a monomeric azulene-functionalised styrene would be co-polymerised with styrene and divinylbenzene to create an azulene-functionalised PS-DVB resin directly (Figure 2.29). However, it was subsequently found that azulene derivatives are highly susceptible to various side-reactions and degradation under radical conditions (see section 2.5.5), rendering this proposed route unfeasible.

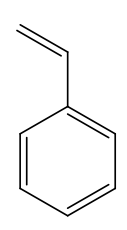

$98-x \%$

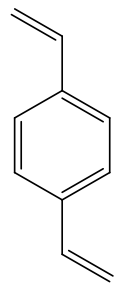

$2 \%$

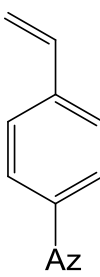

$\mathrm{x} \%$

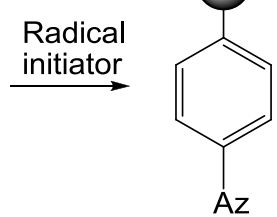

crosslinked

$\mathrm{X} \%$ functionalised

PS-DVB resin

Figure 2.29 Proposed radical initialised polymerisation to form functionalised PS-DVB, with Az representing an arbitrarily attached azulene derivative

An alternative synthetic route must therefore be used. We decided that efforts would be directed towards attaching an azulene moiety to a pre-formed resin.

The first route of inquiry involved the generation of a guaiazulene 3-acyl halide and the subsequent Friedel-Crafts acylation directly on to the phenyl rings present within the resin, as described in section 2.2.3. However since the model reaction involving toluene in the place of PS-DVB could not be successfully performed, this idea was abandoned.

\subsubsection{Synthesis of a guaiazulene-functionalised PS-DVB resin}

An approach to generating a functionalised PS-DVB resin was envisaged that proceeded via the deprotonation of the 4-methyl group of guaiazulene, followed by addition to Merrifield resin. As demonstrated in section 2.3.2, addition of benzyl chloride to the guaiazulenide anion yielded the compound 37. In this case, benzyl chloride served as a useful model for Merrifield resin, as the chloromethylene group is in similar environments for both compounds. This is illustrated in Figure 2.30. 


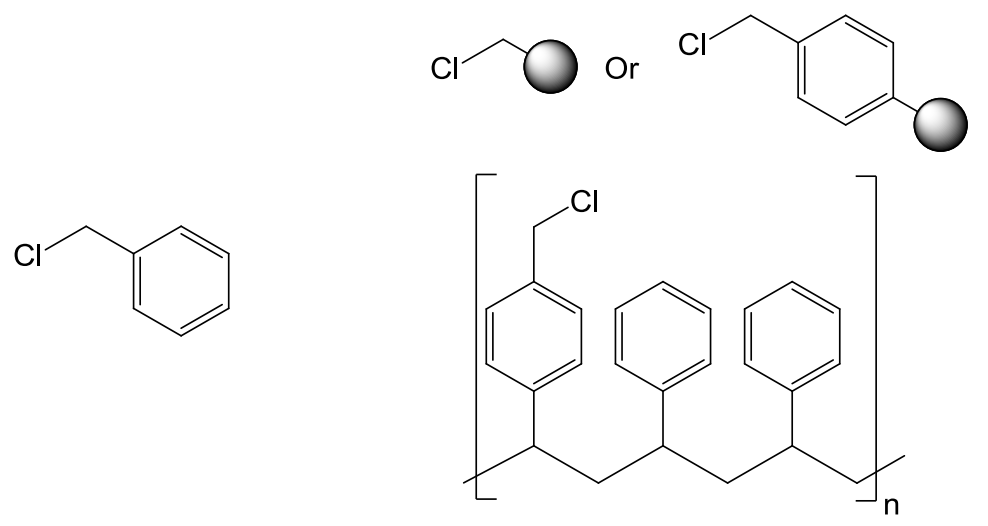

Figure 2.30 Benzyl chloride, left, and three depictions of Merrifield resin, right. The third depiction represents the empirical formula of the particular resin used

A synthesis of the resin $\mathbf{5 0}$ was then carried out under similar conditions to those of the synthesis of $\mathbf{3 6}$ (Figure 2.31).

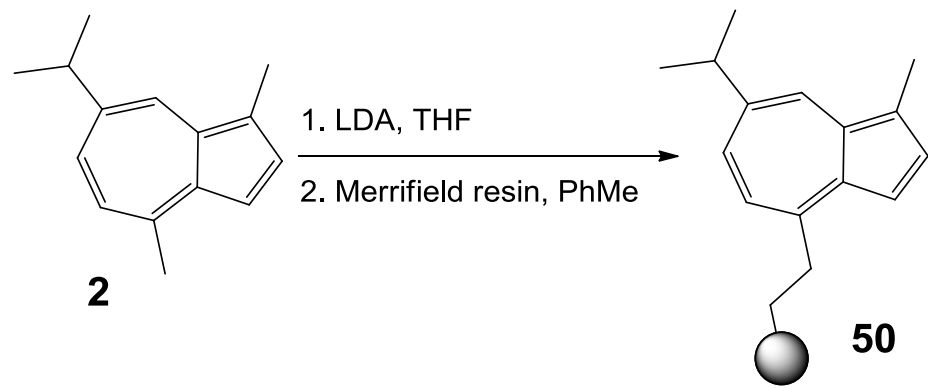

Figure 2.31 Formation of resin $\mathbf{5 0}$

A solution of the guaiazulenide anion in THF was cannulated across to a suspension of Merrifield resin (33\% functionalisation, 2\% cross-linked, 100-200 micron) in toluene. A dark blue-green resin was obtained, whose colour did not fade after copious washing with several solvents (toluene, dichloromethane, acetone, methanol, water), suggesting the guaiazulene moiety was bound chemically to the resin. The appearance of the resin $\mathbf{5 0}$ is shown in Figure 2.32. Analysis of the mass of the leftover guaiazulene residue gives an upper bound of $76 \%$ to functionalisation - that is, at most 76 percent of all chloromethylene groups have been substituted by a guaiazulene. Analysis of the mass of the resin itself gives a different result $-56 \%$ functionalisation. Since removal of all solvent from the resin beads is not always possible, the true level of functionalisation may be lower. Comparing the $56 \%$ level of functionalisation with the yields of similar deprotonation reactions, this result is typical. 


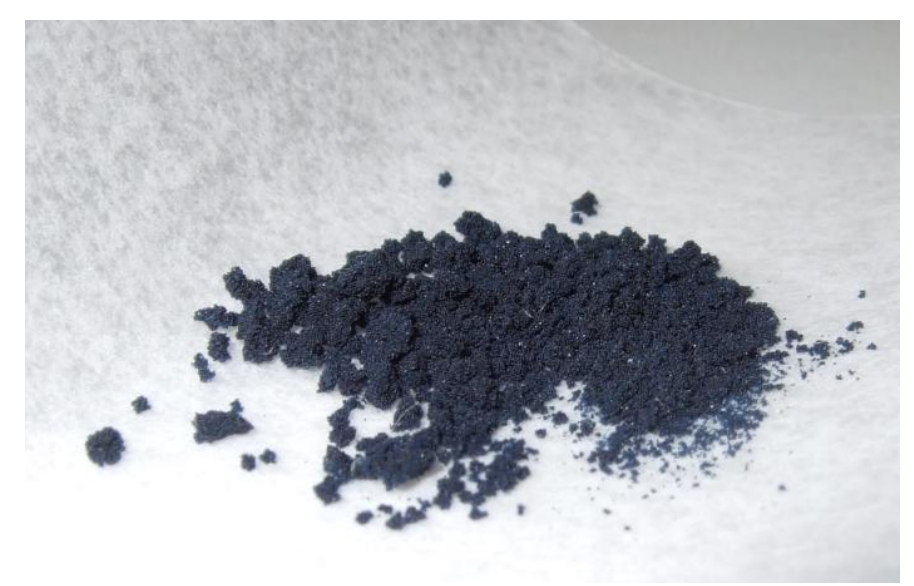

Figure 2.32 Photograph of the guaiazulene resin $\mathbf{5 0}$

\subsubsection{Characterisation of the guaiazulene resin}

Adequate characterisation of this resin was exacerbated by the lack of suitable onresin spectroscopy techniques. The three main techniques in organic chemistry - IR, NMR and Mass Spectrometry - all have difficulties associated with the characterisation of functionalised polymer resins.

FTIR spectroscopy can provide useful information on the nature of the bonding within PS-DVB resins. There are several techniques for IR spectroscopy on resins, including destructive techniques such as grinding with $\mathrm{KBr}$ and non-destructive techniques such as single-bead FTIR microspectroscopy. Aside from baseline tilting caused by lightscattering within the resin, there are few differences between normal and on-resin IR spectroscopy.

IR spectra were obtained for both Merrifield resin and the guaiazulene-functionalised resin 50, using the $\mathrm{KBr}$ grinding method. Both spectra were almost identical, with only a couple of low-intensity peaks absent in the Merrifield spectrum. This is not surprising, as neither the guaiazulene moiety nor the $\mathrm{C}-\mathrm{C}$ bond linking guaiazulene to the resin exhibit readily-identified diagnostic absorbances. Indeed, IR is used most commonly on solid-phase reactions that produce unusual or easily identifiable vibrations. $^{39}$

The use of Mass Spectrometry was ruled out, as the substrate must be cleaved from the resin before ionisation and detection. This can only be possible when the substrate is attached to the resin via a cleavable linker. ${ }^{39}$ Since the resin $\mathbf{5 0}$ does not possess such a linker, MS analysis could not be performed. 
${ }^{1} \mathrm{H}$ NMR spectroscopy was also attempted on the sample. Although PS-DVB resins are usually described as 'solid-phase', in the presence of a highly solvating solvent the resin swells sufficiently to allow 'liquid-like' behaviour to occur within the resin on NMR timescales. The resin $\mathbf{5 0}$ was added to an NMR tube with $\mathrm{CDCl}_{3}$ (a highly solvating solvent) and sonicated to maximise the swelling of the resin. The resulting ${ }^{1} \mathrm{H}$ NMR spectrum consisted of a single peak over $20 \mathrm{ppm}$ wide at half height.

It was since discovered that resins such as $\mathbf{5 0}$ are poorly suited for NMR analysis, due to the small distance between the resin structure and the guaiazulene. ${ }^{39}$

Generally speaking, the broadness of the peaks in a NMR spectrum depends on the degree of freedom of the individual molecules to tumble freely in solution - the less freedom, the broader the peaks. In a highly viscous environment such as the interior of a PS-DVB resin, substantial peak broadening can occur. As the spacing increases between the substrate and the resin, the substrate has more freedom in which to move and consequently the broadening of the peaks is mitigated. Since the guaiazulene moiety is so close to the resin, the NMR spectra exhibit severely broadened peaks, making characterisation through this method impossible with the tools available.

There are NMR experiments that can circumvent this issue. For example, it is possible to obtain meaningful ${ }^{1} \mathrm{H}$ NMR spectra through Magic Angle Spinning. ${ }^{40}$ Otherwise, there are certain other nuclei that be used such as ${ }^{13} \mathrm{C},{ }^{19} \mathrm{~F},{ }^{2} \mathrm{H},{ }^{31} \mathrm{P}$ and ${ }^{15} \mathrm{~N}$, owing to the natural narrowness of their peaks.

\subsubsection{Investigation of on-resin reactions and use as scavenger resins}

Two reactions were subsequently performed on the resin $\mathbf{5 0}$ - Vilsmeier-Haack formylation and acylation using oxalyl chloride (Figure 2.33 \& Figure 2.34). These reactions are analogous to those performed on the compound 37 (refer to Figure 2.22). In both cases, the colour of the resin changed from dark blue to dark brown. Again, the lack of usable spectroscopic methods hindered the characterisation of the resin. The extent of reaction could be approximated by analysis of the mass balance, provided a couple of assumptions are made. 


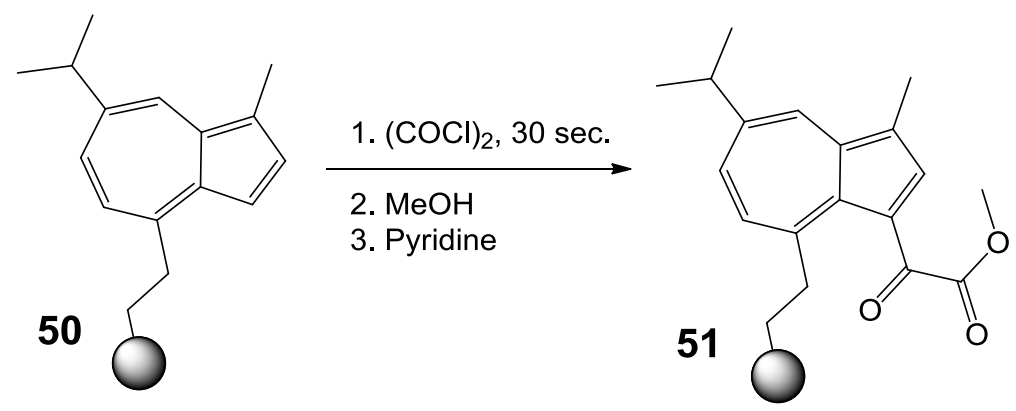

Figure 2.33 Attempted oxalyl chloride acylation on the resin $\mathbf{5 0}$

Following the addition of oxalyl chloride and methanol to 50, an increase of mass from $52.6 \mathrm{mg}$ to $57.1 \mathrm{mg}$ was observed. If the lower estimate for guaiazulene functionalisation of $\mathbf{5 0}$ is used (i.e. $56 \%$, otherwise expressed as the empirical formula $\left.\mathrm{C}_{25} \mathrm{H}_{25}\left(\mathrm{C}_{15} \mathrm{H}_{17}\right)_{0.56} \mathrm{Cl}_{0.44}\right)$, and if it is assumed that the methyl ketoester was the only product from this reaction (i.e. no formation of esters, acids or of diketone dimers), the extent of reaction is approximately $80 \%$. If formation of esters or other products occurred, the extent of reaction will be higher than this value (because esters and other possible functional groups all have lower molecular masses than the methyl ketoester). It is also assumed that there is no net change of the quantity of absorbed material such as solvent or other impurities within the resin.

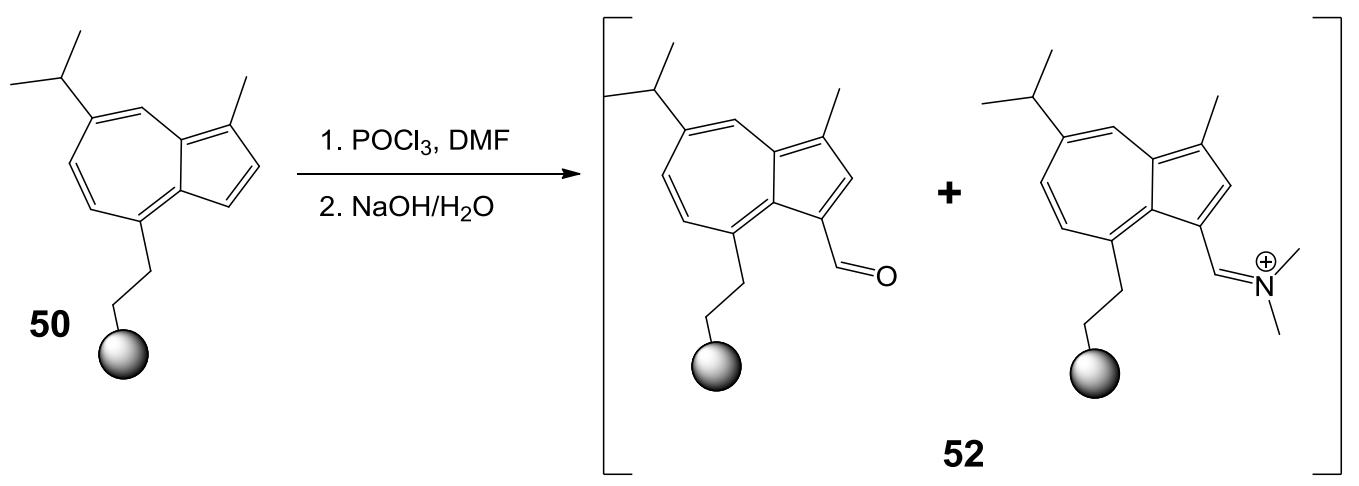

Figure 2.34 Attempted Vilsmeier-Haack formylation of $\mathbf{5 0}$

Similar reasoning was applied to the Vilsmeier-Haack reaction of the resin 50. A change in mass of the resin from $48.4 \mathrm{mg}$ to $49.9 \mathrm{mg}$ was observed. If one assumes the only product of the reaction is the aldehyde, the extent of reaction comes roughly to $89 \%$. If instead the only product is the dimethyliminium species, the extent of reaction comes to $45 \%$. Owing to the likely difficulty of aqueous $\mathrm{NaOH}$ penetrating into the hydrophobic resin to hydrolyse these iminium species, the true degree of functionalisation is likely to lie closer to $45 \%$. Again, this is assuming no net change of solvent or other physically bound compounds occurred during the reaction. 
Despite the difficulties present in characterisation of these compounds, these two reactions are a good demonstration that PS-DVB bound guaiazulene has the potential act as self-indicating scavenger resins for both oxalyl chloride and Vilsmeier reagent, and that self-indicating scavenger resins for other reagents are possible in principle. The system requires further modification before it can easily be used in this way, however. The parent resin itself isn't optimal for use as a scavenger resin. Due to the density of chromophores on this resin, the colour change from blue to brown is very difficult to observe. To circumvent this, a much lower degree of functionalisation is necessary so the intensity of colour is reduced and colour changes can be more readily observed.

\subsubsection{Summary of section 2.4}

A guaiazulene-functionalised PS-DVB resin was synthesised by addition of the guaiazulenide anion to Merrifield resin. Evidence for this is based on the retention of the blue colour after copious washing of the resin with various solvents. Neither IR nor ${ }^{1} \mathrm{H}$ NMR spectra could give any evidence for or against the formation of a guaiazulene-resin bond.

This resin was subjected to Vilsmeier-Haack formylation and acylation with oxalyl chloride. A colour change to brown was noted in both cases, indicating at least partial reaction of the bound guaiazulene with these electrophiles. Analysis of the mass of the products of both reactions, the extent of reaction was estimated to be $80 \%$ in resin $\mathbf{5 1}$ and between $45 \%$ and $89 \%$ in resin $\mathbf{5 2}$. This result demonstrates the potential these resins hold as self-indicating scavenger resins, but there are still issues to be overcome with regards to the resin itself. 


\subsection{Towards development of a guaiazulene-based protecting group system}

\subsubsection{Introduction}

Over the course of this research, numerous azulene-based chemical systems were investigated for properties that could lead to useful protecting groups. In most cases, the compounds involved were either too reactive/prone towards degradation or too elusive in synthesis. In this section all the compounds that were considered as potential protecting groups are discussed, along with their various shortcomings.

\subsubsection{A protecting group for primary amines based on reductive amination and nucleophilic cleavage}

One of the first potential protecting group systems investigated involved the reductive amination of guaiazulene with the appropriate amine substrate.
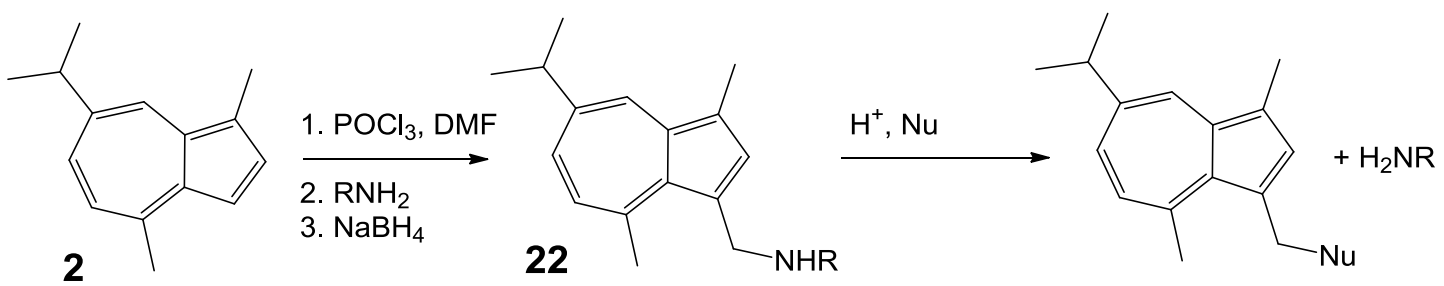

Figure 2.35 First investigated protecting group candidate

As described in detail in section 2.2, the amine $\mathbf{2 2}$ was easily synthesised, but severe problems with the stability of $\mathbf{2 2}$ were identified. Nucleophilic attack on the amine group by the 3-position of another molecule of 22 is believed to occur, resulting in the generation of the bis(guaiazulenyl)methane $\mathbf{2 5}$ and loss of the amine substrate. If this process could be controlled, there is still potential here for a useful protecting group, but not without significant modification of the azulene ring.

\subsubsection{A protecting group for cis-diols based on 3-formyl guaiazulene}

The protection of cis-diols was an obvious application for the aldehyde 19. In a manner similar to acetonide or benzylidine protecting groups, a 3-formylazulene would be reacted with an unprotected diol in the presence of an acid to generate an acetal (Figure 2.36), and addition of aqueous acid would then cleave the protecting group and regenerate the diol. 


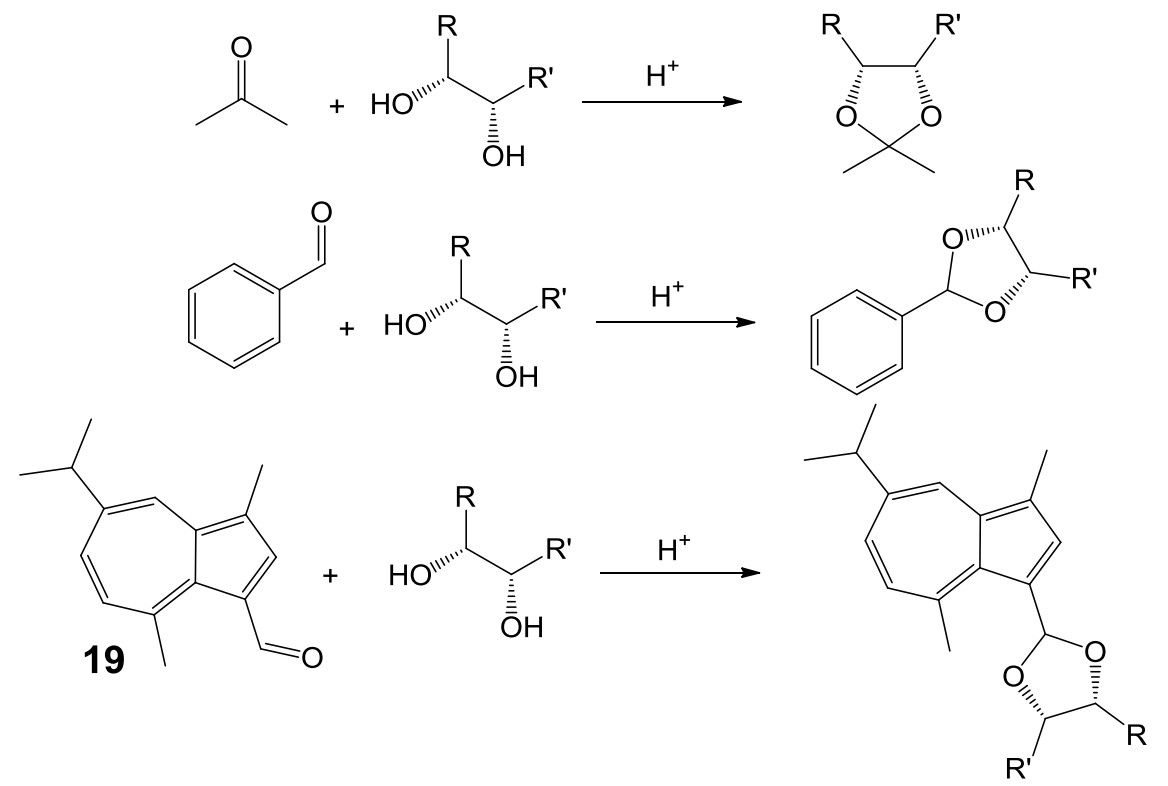

Figure 2.36 From top, acetonide and benzylidene diol protecting groups and the proposed guaiazulene analogue, bottom

This system was also predicted to feature a colour change, as the aldehyde/acetal functional group is directly attached to the azulene moiety.

A few attempts were made to synthesise the dimethyl acetal of the aldehyde 19, using $p$-toluenesulfonic acid and trimethyl orthoformate. These reactions returned mostly starting material and a small quantity of an unidentified red compound with a tendency to degrade rapidly.

A paper by W. Treibs et al. ${ }^{41}$ was later discovered in which synthesis of this exact acetal had been attempted. Here too, it was found that this acetal would degrade rapidly, although a possible reason for this was not specified. This instability prohibited the aldehyde $\mathbf{1 9}$ from being used as a protecting group for diols.

\subsubsection{An FMOC-analogous protecting group}

We hypothesised that the acidity of the proton on the 4-methyl position would allow E1 or E2-type elimination reactions to take place on the alpha position, in a manner similar to the deprotection mechanism of the FMOC protecting group.

The FMOC group (fluorenylmethyloxycarbonyl) is a popular protecting group in peptide chemistry, owing to its effective protection of amines and mild deprotection conditions. $^{42}$ The protection step of an amine with $\mathrm{FMOC}-\mathrm{Cl}$ is illustrated in Figure 2.37. 

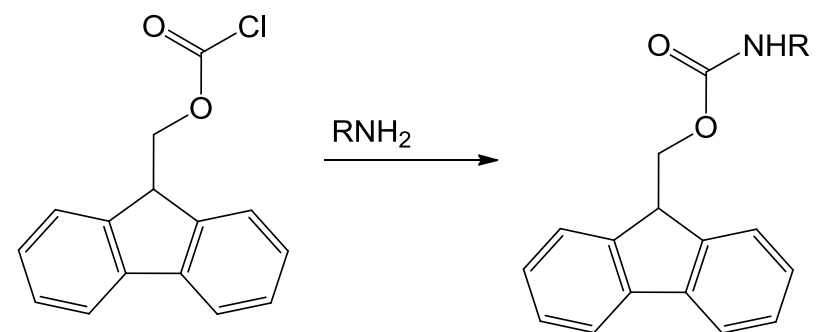

53

Figure 2.37 FMOC-Cl, left and an FMOC-protected amine, right

In particular, the deprotection conditions of FMOC involve the addition of a mild base to deprotonate the 9- position on the fluorene structure, resulting in an elimination of the carbamate group, depicted in Figure 2.38. The proton at the 9- position is slightly acidic, due to the cyclopentadienide anion in the conjugate base. This reaction is pushed to completion not only by the formation of the conjugated dibenzofulvene $\mathbf{5 4}$, but also through the evolution of carbon dioxide.<smiles>[R]NC(=O)C(C)C(=O)N[R]</smiles>

Figure 2.38 Deprotection mechanism of FMOC with mild base

It was postulated that a similar elimination reaction (depicted in Figure 2.39) may be possible on a guaiazulene derivative due to the acidity of the 4-methyl group.

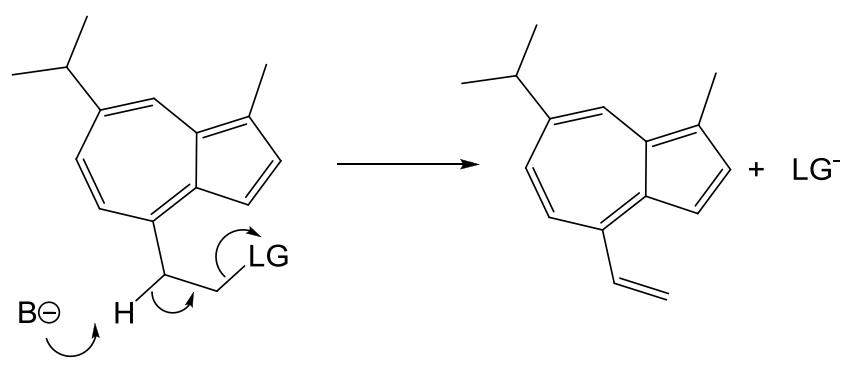

Figure 2.39 Postulated deprotection mechanism of a FMOC-analogous azulene-based protecting group As a proof of concept, the ester $\mathbf{5 5}$ was synthesised and added to a solution of piperidine in DMF and left overnight, conditions similar to that used in FMOC deprotection (Figure 2.40). 


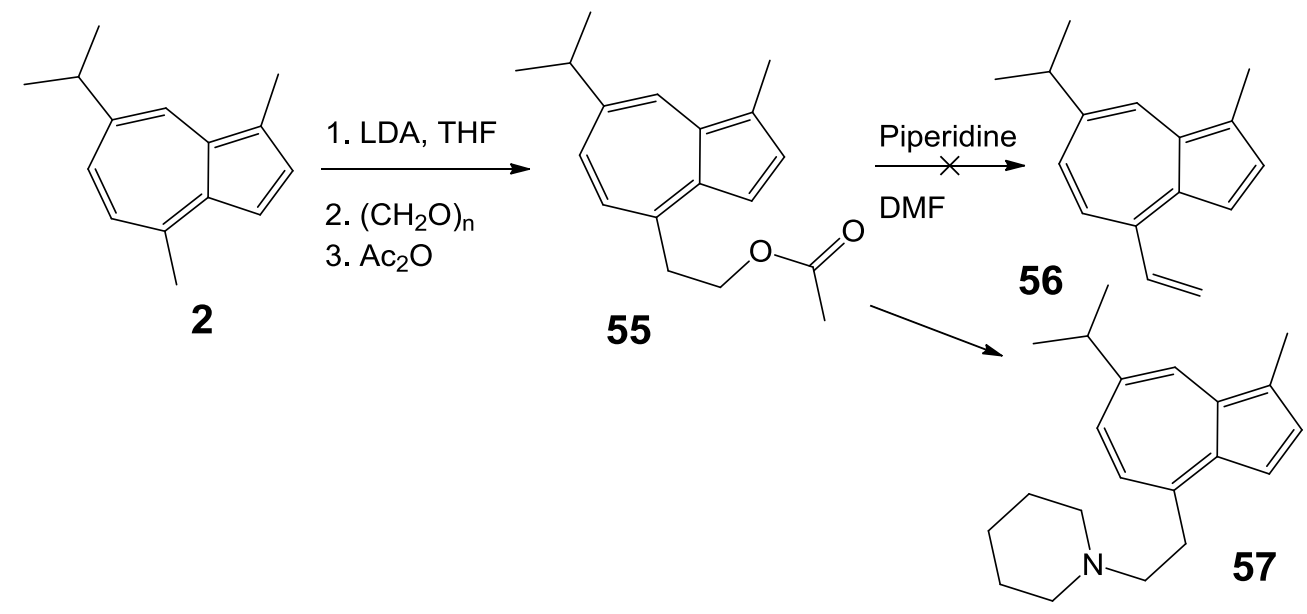

Figure 2.40 The ester $\mathbf{5 5}$ was synthesised and reacted with piperidine/DMF to form $\mathbf{5 7}$

However, the expected alkene was not observed. The identity of the reaction product was discovered to be the compound 57, whose structure was confirmed through observation of an HMBC coupling between the piperidine ring and the 4-ethylene chain. The formation of this product is presumably due to the guaiazulene moiety polarising the double bond through resonance, causing the distal end to be electrophilic. The piperidine, being a nucleophilic base, inserts itself into this double bond to create the amine 57. Piperidine inserts itself into dibenzofulvene in a similar manner when it is used as a base in FMOC deprotection. ${ }^{42}$

In any case, this result shows that a leaving group such as acetate can be removed in an elimination reaction with the use of a mild base. If removal was due to nucleophilic attack on the ester, one would expect the presence of the alcohol 36 in the product mixture, which was not observed. At this point, we hypothesised that this chemistry could be further expanded by the addition of an electron-withdrawing group such a methyl ester or an aldehyde at the 3-position. This would further increase the acidity of the 4-methylene position, resulting in a two-step deprotection whose second step could be possible under even milder conditions.

At the time, this was an exciting result that demonstrated the potential for a useful coloured protecting group. However, a paper describing these ideas was released simultaneously. ${ }^{43}$ In this paper by Aumüller et al. an analogous amine protecting group to FMOC was developed. The alcohol 36 was synthesised as described in their previous paper. ${ }^{16}$ To this disuccinimidyl carbonate was added to produce the carbonate 58, and reacted with an amine in the presence of DIPEA in DMF to produce carbamate 59 in an $85 \%$ yield (based on the starting amine) (Figure 2.41). 

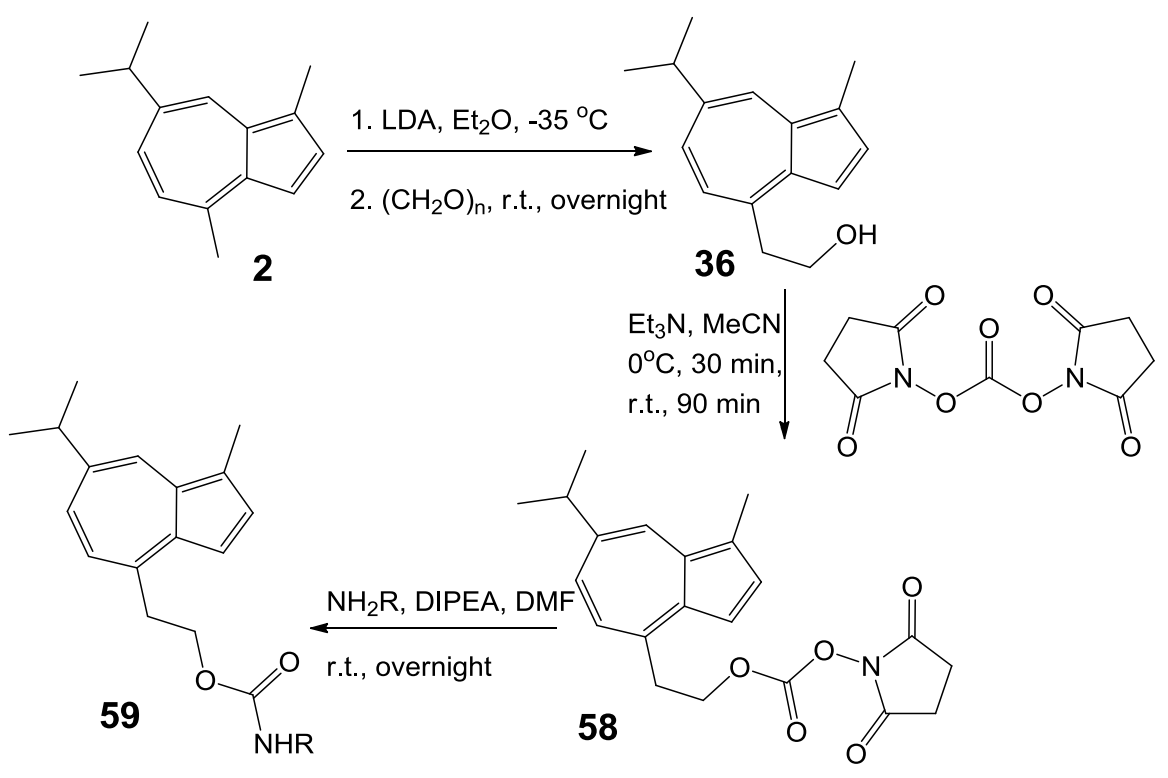

Figure 2.41 Synthesis of carbamate 59, as presented in the paper by Aumüller et al.

Subsequent deprotection involved the addition of lithium thioethanolate, a compound with intermediate basicity between alkoxides and amines. This deprotection was achieved with a high yield of $72 \%$. It should be noted that in this work, it was observed that the thioethanolate base also inserted into the double bond of the vinylogous azulene to produce $\mathbf{6 1}$, behaviour which is in agreement with our observations (Figure 2.42).

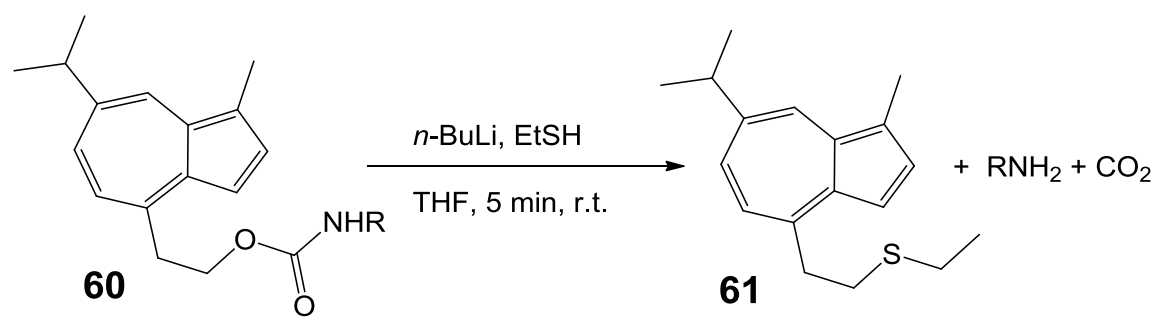

Figure 2.42 Deprotection of the carbamate with lithium thioethanolate affords $\mathbf{6 1}$ and the amine substrate in high yield

Furthermore, this paper goes on to demonstrate that deprotection can indeed be made milder through addition of an electron withdrawing group at the 3-position. Instead of an aldehyde or ester, a nitro group was used for this purpose (Figure 2.43).

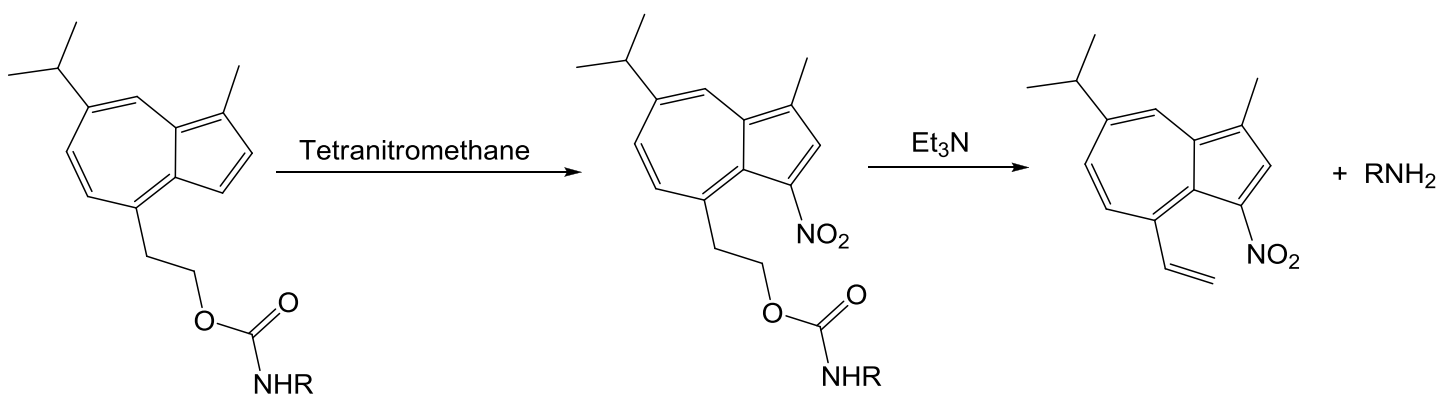

Figure 2.43 Nitration of the 3-position allows deprotection to take place in much milder conditions 
By adding a nitro group, achieved through use of the nitrating agent tetranitromethane, the elimination reaction can be catalysed even by mild bases such as $\mathrm{Et}_{3} \mathrm{~N}$.

Although the protecting group described in the paper is not exactly the same as ours, it was deemed sufficiently similar that research on this line was discontinued. This paper, however, vindicated many of the ideas that had been proposed in this research, and served as a useful springboard for further work.

\subsubsection{Towards synthesis of a base-labile diol protecting group}

We sought to find other protecting group applications utilising the unusual acidity of the 4-methyl proton. One such application returned to a guaiazulene aldehyde as a protecting group for diols. A paper by T. Fukuyama et al. ${ }^{44}$ was discovered wherein the cleavage of a para-silyl ether benzylidene acetal protecting group was performed under basic conditions with addition of fluoride, depicted in Figure 2.44.<smiles>[R]C1OC(c2ccc(O[R16]([H])([H])[NH3+])cc2)OC1[R]O[Na]</smiles>

Figure 2.44 Base-mediated deprotection of diols

Upon generation of the para-alkoxide through removal of the TBS group, a lowenergy route to the aldehyde is set up, allowing the diol to be recovered in $93 \%$ yield.

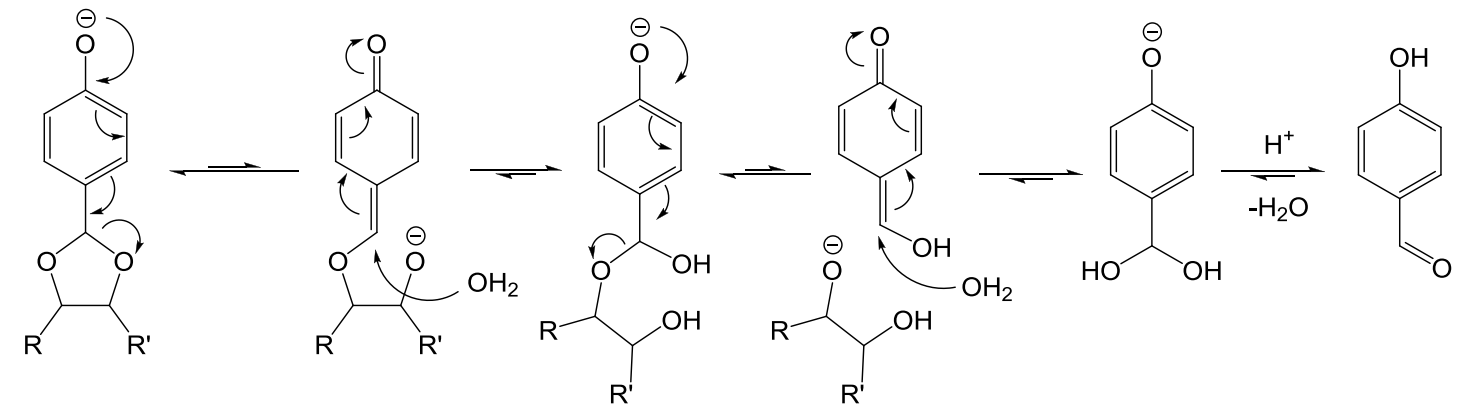

Figure 2.45 Proposed mechanism of deprotection of the acetal 
It was proposed that this reactivity could be replicated by the acidic proton on the 4methyl position of guaiazulene. The compound 63 would be the ideal target for this, as deprotection of the corresponding acetal was anticipated to follow the mechanism in Figure 2.46.

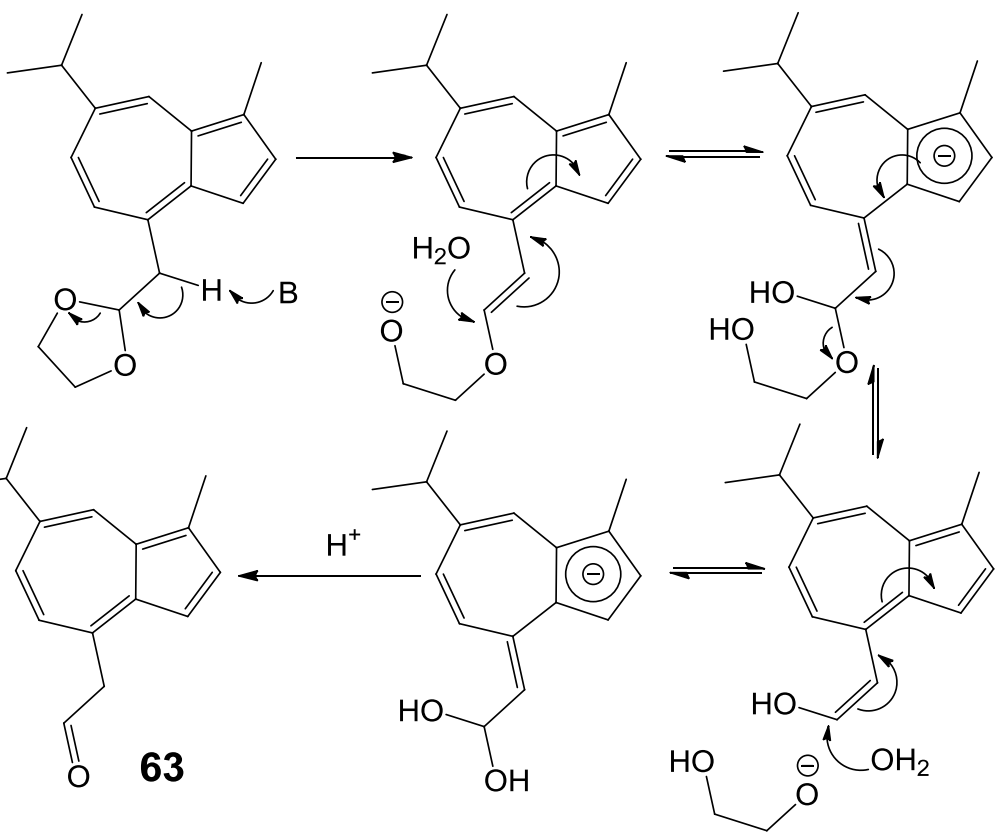

Figure 2.46 Postulated cleavage mechanism of a guaiazulene analogue of 62

Several routes were proposed towards the synthesis of 63. The first attempt at synthesising this compound involved addition of ethyl formate to the guaiazulenide anion. Not surprisingly, guaiazulene inserted itself twice into the carbonyl to form the dimeric alcohol 64 in $29.7 \%$ yield and its formate ester $\mathbf{6 5}$ in $32.4 \%$ yield (Figure 2.47). Most importantly, the presence of these two products implies the existence of the desired compound $\mathbf{6 3}$ as an intermediate.

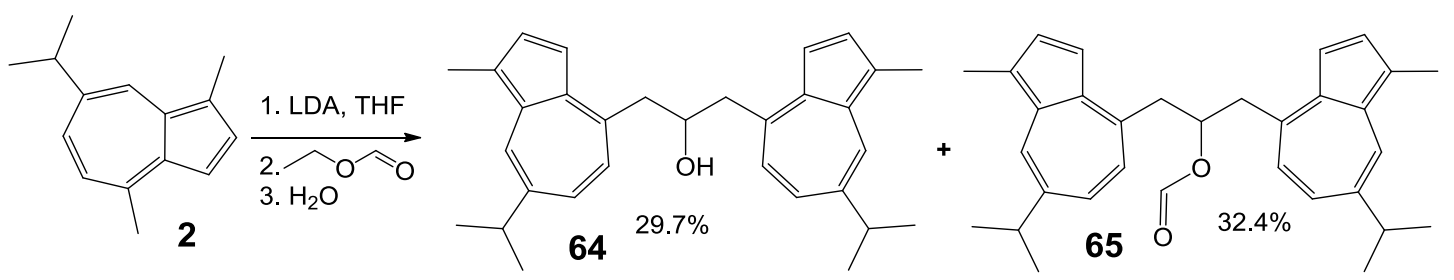

Figure 2.47 Addition of ethyl formate to the guaiazulenide anion

The dimeric nature of these compounds was identified through the ${ }^{1} \mathrm{H}$ NMR integration of the central methine and formate proton for $1 / 2 \mathrm{H}$. These structures were later confirmed through Mass Spectrometry.

This reaction was attempted a second time with the same reagents, but instead of adding ethyl formate to the reaction mixture, the reaction mixture itself was cannulated across into another flask containing $1 \mathrm{~mL}$ of THF and $0.6 \mathrm{~mL}(6.09$ 
equivalents) of ethyl formate, to ensure ethyl formate is in excess at all times during reaction.

As before, this reaction produced the alcohol and formate ester in $12.3 \%$ yield and $16.6 \%$ yield respectively, along with a larger than usual quantity of unidentifiable brown-green degradation products. These results suggest the rate of nucleophilic substitution by the second guaiazulenide is much greater than that of the first substitution, possibly suggesting the aldehyde intermediate is relatively high-energy. This reaction was repeated a third time with 74 equivalents of ethyl formate with similar results again.

Various other routes to 63 or the ketone analogue 66 were tried (Figure 2.48). DMF commonly used in Grignard chemistry to obtain aldehydes - was added to a solution containing the guaiazulenide anion. After addition, no colour change was observed. After purification only guaiazulene and degradation products were present. Similar results were obtained when acetonitrile - whose reactivity towards Grignard reagents is similar to DMF - was used. Only guaiazulene and degradation products were present after purification.

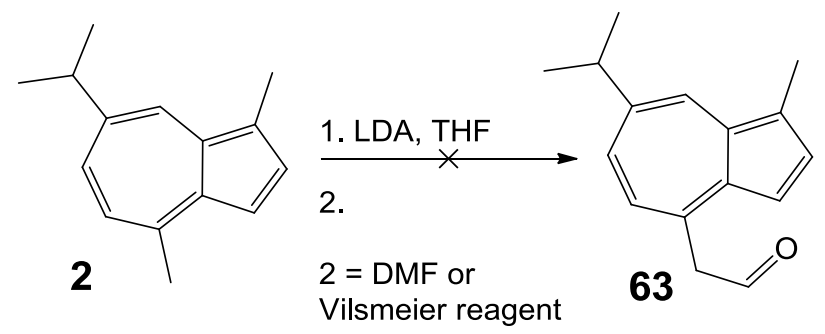

Figure 2.48 Synthesis of 63 using the electrophiles DMF and Vilsmeier reagent

Even more aggressive approaches failed. Out of curiosity, the chloroiminium ion normally used in Vilsmeier-Haack formylation was added to the deprotonated guaiazulene, whereupon a slight colour change from one shade of orange to another was observed. This orange colour was still present during workup, and after column chromatography was performed the only identifiable product was the aldehyde 19, isolated in $37 \%$ yield.

Acetic anhydride was also tried as an electrophile, with the intention of creating the ketone 66, instead of the aldehyde 63. This reaction appeared more promising, as a colour change from orange to blue was observed upon addition of acetic anhydride. A 
product was obtained in relatively high yield ( $41 \%$ by mass) that was neither guaiazulene nor degradation products. The ${ }^{1} \mathrm{H}$ NMR spectrum gave circumstantial evidence for the intended product (a methylene at $3.6 \mathrm{ppm}$ was present, but the carbonyl $\alpha$-methyl was conspicuously absent). However, ${ }^{13} \mathrm{C}$ NMR conclusively showed the absence of a carbonyl, and degradation took place before further analysis could be performed.

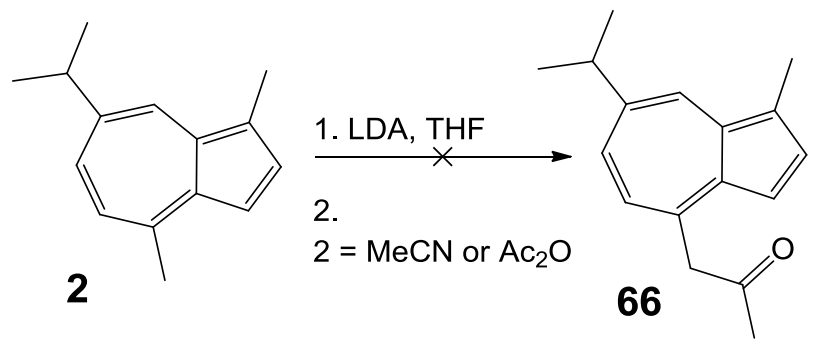

Figure 2.49 The electrophiles $\mathrm{MeCN}$ and $\mathrm{Ac}_{2} \mathrm{O}$ failed to produce the ketone $\mathbf{6 6}$

Alternate ways of preparing the aldehyde were sought. TEMPO-BAIB oxidation of the alcohol 36 was attempted (Figure 2.50).

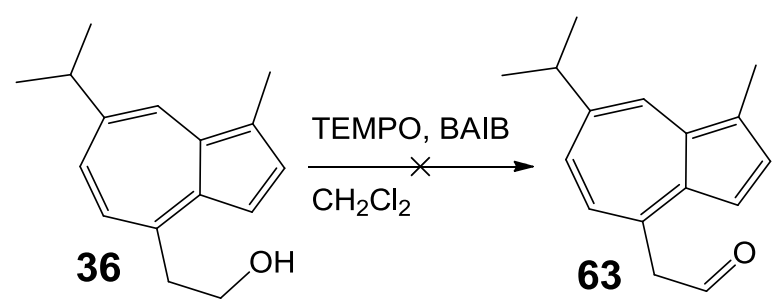

Figure 2.50 TEMPO-BAIB oxidation of $\mathbf{3 6}$ failed to produce the aldehyde $\mathbf{6 3}$

The radical conditions present in this reaction were highly effective at degrading the azulene moiety - after 30 minutes the reaction mixture had become grey and no identifiable products could be obtained from it.

Swern oxidation was also attempted on the alcohol (Figure 2.51), but instead of obtaining the aldehyde, a purple, highly water-soluble sulfonium cation was obtained in an inseparable mixture with $\mathrm{NEt}_{3}$ and DMSO.

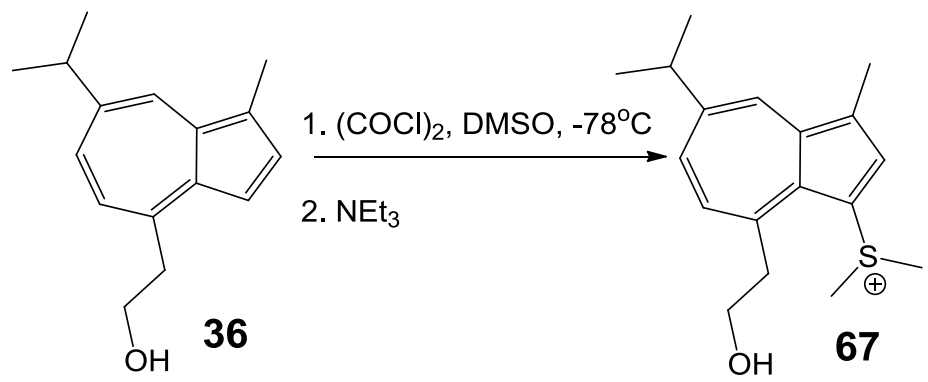

Figure 2.51 Attempted Swern oxidation of $\mathbf{3 6}$ resulted in the sulfonium cation $\mathbf{6 7}$ 
Swern oxidation involves the addition of oxalyl chloride and DMSO to create the electrophilic dimethylchlorosulfonium ion.

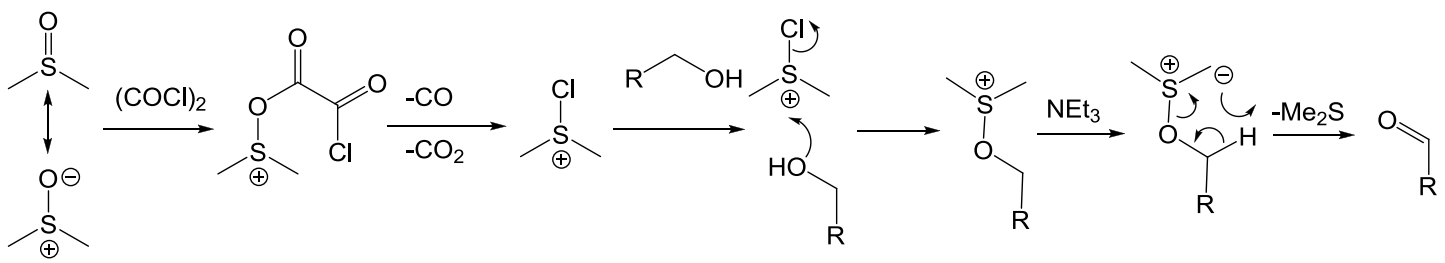

Figure 2.52 Mechanism of Swern oxidation

In ordinary function, the chlorosulfonium cation is attacked nucleophilically by an alcohol, and upon addition of a base one of the methyls on the sulfonium moiety is deprotonated, abstracting a carbinol proton and generating the aldehyde along with $\mathrm{Me}_{2} \mathrm{~S}$ gas.

In the case depicted in Figure 2.51, the nucleophilic 3- position on the guaiazulene structure reacted preferentially with the chlorosulfonium ion, generating the sulfonium in large quantity. As is usual for cations at the 3-position of guaiazulene, the sulfonium ion is stabilised by resonance with the tropylium structure. There was no evidence of any reaction on the hydroxyl group. This sulfonium chemistry was briefly investigated on guaiazulene itself.

The Swern oxidation reaction conditions were applied to guaiazulene to produce the guaiazulene sulfonium cation 68 (Figure 2.53), but isolation proved to be problematic, as the purple sulfonium ion would degrade to a brown substance during purification. Eventually, it was found that presence of $\mathrm{NEt}_{3}$ was necessary during the reaction in order to prevent decomposition, presumably through stabilisation of the cation by complexation of the amine to the sulfonium ion. The trade-off of this was complete removal of $\mathrm{NEt}_{3}$ was no longer possible even under high vacuum and an accurate yield of 67 could not be obtained. Due to the difficulty in purification and in obtaining clear NMR spectra, only circumstantial evidence (such as the purple colour, water solubility and some peaks in the ${ }^{1} \mathrm{H}$ NMR spectrum) for formation of $\mathbf{6 8}$ was obtained.

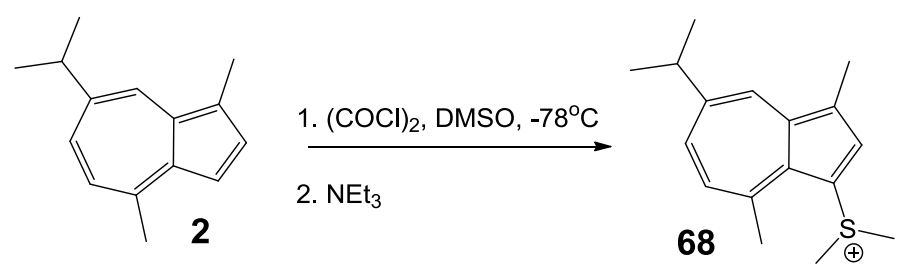

Figure 2.53 Synthesis of the guaiazulene 3-sulfonium cation 68 
Nevertheless, LDA was added to the sulfonium 68, primarily out of curiosity of which site would deprotonate preferentially, but upon addition of LDA an unidentifiable brown-orange colour was produced. This species did not appear to be the analogue of the guaiazulenide anion, as no colour change upon addition of $\mathrm{MeOH}$ occurred.

The aldehyde 63 remains an elusive target. It is not clear what the properties of this compound would be upon synthesis. The acidity of the 4-methylene proton is expected to be rather high, comparable to that of a $\beta$-diketone, owing to the presence of both cyclopentadienide and enolate resonance forms (Figure 2.54). Whether or not this compound can be deprotonated by bases such as sodium hydroxide remains to be seen.
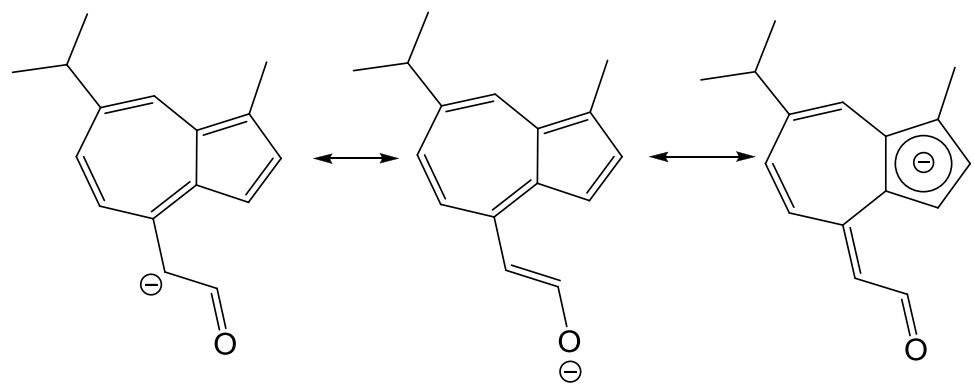

Figure 2.54 Resonance structures of 63 upon deprotonation

A brief computational study was undertaken to assess the relative stability of the aldehyde with respect to the cis- and trans-enol forms (see section 2.6.3 for more details). It was found that the aldehyde is between $50-100 \mathrm{~kJ} \mathrm{~mol}^{-1}$ more stable than either enol tautomer, depending on the parameters used in calculation.

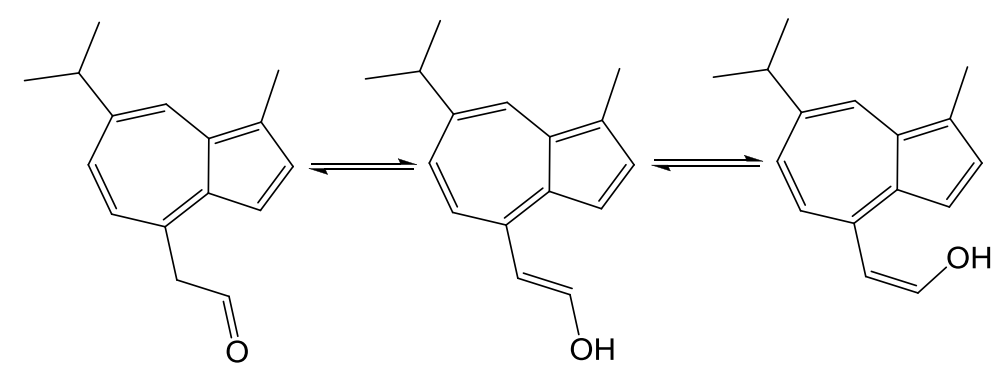

Figure 2.55 The aldehyde 63 and its cis- and trans- enol tautomers

The synthetic routes to the compound $\mathbf{6 3}$ or derivatives are by no means exhausted, however single-minded pursuit of this compound was becoming less attractive as other potential protecting groups warranted investigation. 


\subsubsection{Towards the synthesis of an azulenic silyl protecting group}

Silyl protecting groups are a popular means of protecting hydroxyl groups, as not only do they afford an efficient way of protecting hydroxyl groups, but the variation in construction and bulkiness between silyl groups results in a graduated lability to acidic and basic conditions. This often allows different silyl groups attached to the same molecule to be cleaved selectively by tuning the strength of the acid or base used.

As a protecting group, an azulenic silyl group would be one more tool in the arsenal of synthetic chemists, while having the obvious advantage of being coloured. Unlike the potential protecting groups $\mathbf{5 5}$ and $\mathbf{6 3}$ discussed earlier, the electronic properties of the azulene moiety are unlikely to have a major effect on the reactivity or lability of the silyl ether moiety. Conversely, the silyl group is expected to have little effect on the electronics of the guaiazulene moiety, resulting in no expected colour change upon addition and cleavage (Figure 2.56).

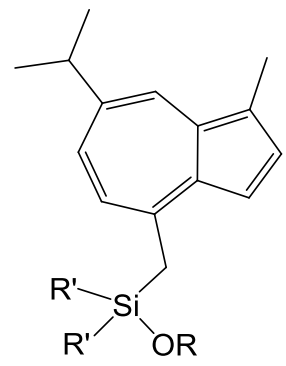

Figure 2.56 General form of a guaiazulene-functionalised silyl ether protecting group

By varying the nature of the other two alkyl groups attached to the silane (for example, $\left.\mathrm{R}^{\prime}=\mathrm{Me}, \mathrm{Ph},{ }^{\mathrm{t}} \mathrm{Bu}\right)$, further variation in reactivity may be obtained.

In order to investigate the reactivity of the guaiazulenide anion with silyl chlorides, trimethylsilane was chosen as a relatively simple reagent. This was added to a solution containing the guaiazulenide anion, and the blue silane 69 was obtained in $67 \%$ yield after purification (Figure 2.57). HMBC coupling between the 9 protons on the TMS group and the carbon at the 4-methylene position gives evidence for this structure, which was confirmed by the correct molecular mass obtained by Mass Spectrometry. 


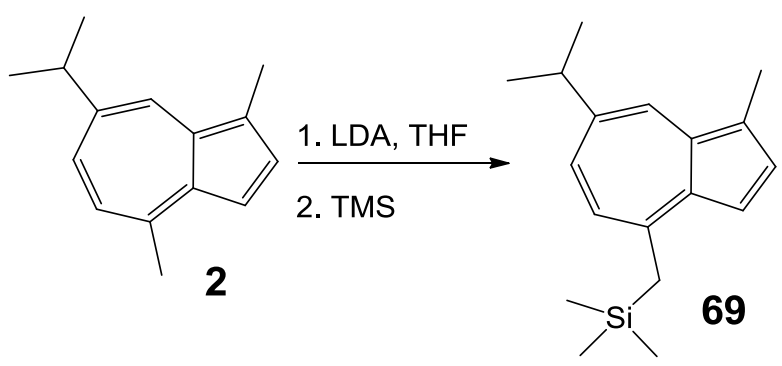

Figure 2.57 Synthesis of the trimethylsilyl derivative 69

The design of the protecting group system involved the addition of dichlorodimethylsilane to the guaiazulenide anion to create a mono azulenesubstituted chlorosilane before being reacted with the appropriate alcohol to create the silyl ether protecting group $\mathbf{7 0}$ (Figure 2.58).

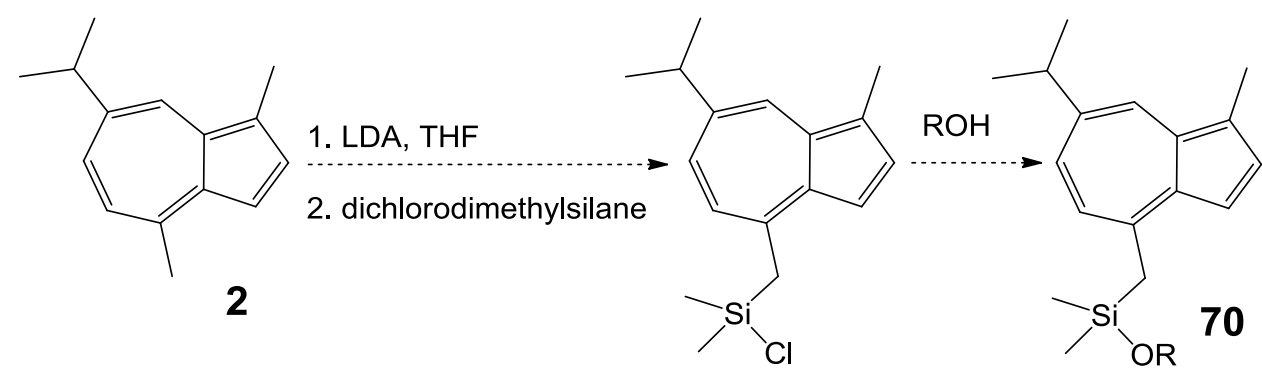

Figure 2.58 Proposed route to the silyl ether $\mathbf{7 0}$

Surprisingly, many difficulties were encountered with this reaction. Both chlorotrimethylsilane and dichlorodimethylsilane produce a rapid colour change from orange to blue when added to a solution containing the guaiazulenide anion. However after addition of dichlorodimethylsilane, the starting material was always recovered in high yields, indicating the presence of acidic compounds in the dichlorodimethylsilane reagent. Subsequent attempts at purifying this silane through distillation before use also resulted in recovery of starting material. Even after distillation of the silane over sodium wire this was observed. Finally, zirconocene dichloride was trialled as a catalyst for this reaction with the same results again.

At this point, no more time was available to further investigate this reaction. It is unclear exactly why no reaction between the guaiazulenide anion and dichlorodimethylsilane took place. Grignard reactions on dichlorodimethylsilane have been reported multiple times in literature. ${ }^{45,46}$ The particular batch of silane used was 95\% pure by NMR. If one assumes that for every $1 \mathrm{~mol}$ of dichlorodimethylsilane hydrolysed, $4 \mathrm{~mol}$ of acidic compounds result (two equivalents of hydrogen chloride plus one equivalent of a silanediol), this only accounts for a $20 \%$ concentration by 
mole of acidic groups - not enough to explain the complete and near-instantaneous reprotonation of guaiazulenide observed.

\subsubsection{Summary of section 2.5}

Various candidates for protecting group systems were investigated. As described in part 1, the amine 22 exhibited reactivity with itself in the presence of mild acid, making this compound unsuitable as part of a protecting group system. Acetal derivatives of the aldehyde $\mathbf{1 9}$ were found to be unstable and difficult to synthesise.

Perhaps the most promising protecting group was the FMOC-analogous system 55. Protection of an acetate group and cleavage under mild conditions was demonstrated. Unfortunately, a paper by Aumüller et al. describing a very similar FMOC-analogous protecting group for amines was made available, detailing several ideas that had already been proposed in our research. As such, other protecting group systems utilising similar chemistry were sought.

One such system involved aldehyde $\mathbf{6 3}$ being a base labile protecting group for cisdiols. Various attempts were made at making 63 , with occasionally interesting results but no success.

Finally, the synthesis of a guaiazulene-functionalised silyl ether was attempted. Although facile reaction of the guaiazulenide anion with trimethylsilyl chloride was observed, isolation of any products formed by reaction with dichlorodimethylsilane was not achieved. 


\subsection{Computational results}

\subsubsection{Introduction}

In addition to experimental work, various calculations on azulene derivatives were performed in order to gain a better understanding of their properties.

All calculations were performed using the computational chemistry package Spartan'08 ${ }^{\mathrm{TM}}$ v. 1.1 .1 for Windows on an Intel ${ }^{\circledR}$ dual core $2.66 \mathrm{GHz}$ computer with 2 GB RAM.

\subsubsection{Calculation of the visible absorption spectra of azulene derivatives}

As discussed in the introduction, different functional groups affect the colour of the azulene moiety in different ways, depending on their position on the azulene ring and whether they are electron-donating or electron-withdrawing. Experimentally, evidence for this has been reported in the variation of the colours of various methylazulene derivatives, a well as the colours observed for various derivatives at the 1- and 3- positions. To the author's knowledge, there has been no systematic computational study of a range of derivatives on all positions on the azulene structure.

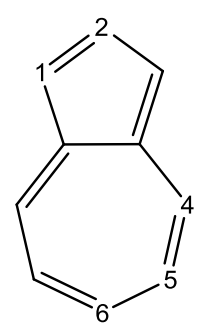

Figure 2.59 The positions on the azulene structure on which calculations were performed

To explore how far the observed patterns extend, a series of geometry optimisations and excited state calculations were performed on azulene and amino-, hydroxy-, methyl-, vinyl-, fluoro-, trifluoromethyl-, methyl ester-, formyl-, trifluoromethyl ketone-, cyano-, nitro- and methyl ketoester- derivatives at all five unique positions on the azulene ring (As labelled in Figure 2.59). Geometry optimisation calculations were performed at the DFT level of theory, using the EDF2 functional and the 6$31 \mathrm{G}^{* *}$ basis set. Excited-state calculations were obtained using TDDFT using the same basis set and functional. Most of the absorption peaks occur within the ultraviolet region, including the prominent $S_{0}-S_{2}$ transition. These peaks tend not to be 
heavily affected by the presence of these groups. The $S_{0}-S_{1}$ transition occurs in the visible region and is heavily dependent on the nature and position of the substituents.

The visible absorbance of azulene itself is calculated to be $492.4 \mathrm{~nm}$. The calculated absorbances of the derivatives are listed in Table 2.1.

\begin{tabular}{|l|r|r|r|r|r|}
\hline & \multicolumn{1}{|l|}{1 - } & \multicolumn{1}{l|}{ 2- } & \multicolumn{1}{l|}{4 - } & \multicolumn{1}{l|}{5 - } & \multicolumn{1}{l|}{6 - } \\
\hline Amino & 628.2 & 433.8 & 450.9 & 547.7 & 456.7 \\
\hline Hydroxyl & 584.1 & 441.7 & 452.5 & 534.4 & 463.3 \\
\hline Vinyl & 557.8 & 513.0 & 522.5 & 507.0 & 537.0 \\
\hline Methyl & 518.2 & 484.0 & 491.3 & 501.5 & 489.3 \\
\hline Methyl ester & 479.4 & 520.9 & 511.4 & 483.6 & 553.9 \\
\hline Formyl & 486.5 & 547.7 & 578.8 & 486.1 & 577.8 \\
\hline Trifluoromethyl & 482.8 & 500.5 & 522.2 & 493.3 & 513.3 \\
\hline Trifluoromethyl ketone & 473.0 & 565.2 & 582.4 & 482.3 & 614.6 \\
\hline Nitro & 467.9 & 543.9 & 608.3 & 483.5 & 599.8 \\
\hline Fluoro & 537.1 & 453.2 & 476.1 & 516.7 & 474.2 \\
\hline Cyano & 501.0 & 512.8 & 545.5 & 496.1 & 541.6 \\
\hline Methyl ketoester & 477.3 & 592.7 & 591.1 & 477.9 & 606.9 \\
\hline
\end{tabular}

Table 2.1 List of $S_{0} \rightarrow S_{1}$ transition values $(\mathrm{nm})$ for various monosubstituted azulenes at positions 1-6

In this table, there are some clear examples of the rule described in section 1.2.3. For instance, 1-aminoazulene has a transition at the long wavelength of $628.2 \mathrm{~nm}$, whereas 4-aminoazulene has a transition at the much shorter wavelength of 450.94 $\mathrm{nm}$. These values are reversed for the ketoester derivative.

Greater insight can be found by finding the difference between these values and unsubstituted azulene and plotting them against the Hammet $\sigma_{\mathrm{p}}$ values for the substituents. The $\sigma_{\mathrm{p}}$ values represent the effect of para-substituents on the pKa of benzoic acid and include both resonance and inductive effects, and were obtained from a review by Taft and co-workers. ${ }^{47}$ Unfortunately the $\sigma_{\mathrm{p}}$ constant for the methyl ketoester substituent could not be found, so was excluded from Table 2.2.

\begin{tabular}{|l|r|r|r|r|r|r|}
\hline & \multicolumn{1}{|l|}{$1-$} & \multicolumn{1}{l|}{ 2- } & \multicolumn{1}{l|}{ 4- } & \multicolumn{1}{l|}{$5-$} & \multicolumn{1}{l|}{6} & $\sigma_{\mathrm{p}}$ \\
\hline Amino & \multicolumn{1}{|l}{135.72} & -58.6 & -41.49 & 55.3 & -35.73 & -0.66 \\
\hline Hydroxyl & 91.63 & -50.72 & -39.9 & 41.99 & -29.09 & -0.37 \\
\hline Methyl & 25.72 & -8.46 & -1.1 & 9.04 & -3.18 & -0.17 \\
\hline Vinyl & 65.39 & 20.6 & 30.06 & 14.52 & 44.53 & -0.04 \\
\hline Fluoro & 44.67 & -39.23 & -16.33 & 24.27 & -18.23 & 0.06 \\
\hline Formyl & -5.98 & 55.25 & 86.35 & -6.33 & 85.4 & 0.42 \\
\hline Methyl ester & -13.03 & 28.47 & 18.95 & -8.83 & 61.47 & 0.45 \\
\hline Trifluoromethyl & -9.59 & 8.07 & 29.78 & 0.84 & 20.85 & 0.54 \\
\hline Cyano & 8.55 & 20.37 & 53.07 & 3.67 & 49.17 & 0.66 \\
\hline Nitro & -24.52 & 51.45 & 115.86 & -8.89 & 107.38 & 0.78 \\
\hline Trifluoromethyl ketone & -19.42 & 72.78 & 90 & -10.13 & 122.21 & 0.8 \\
\hline
\end{tabular}

Table 2.2 Deviation of the $S_{0} \rightarrow S_{1}$ absorbance values from that of azulene 
A graph of these values for each position was plotted against the Hammett parameter of each substituent.

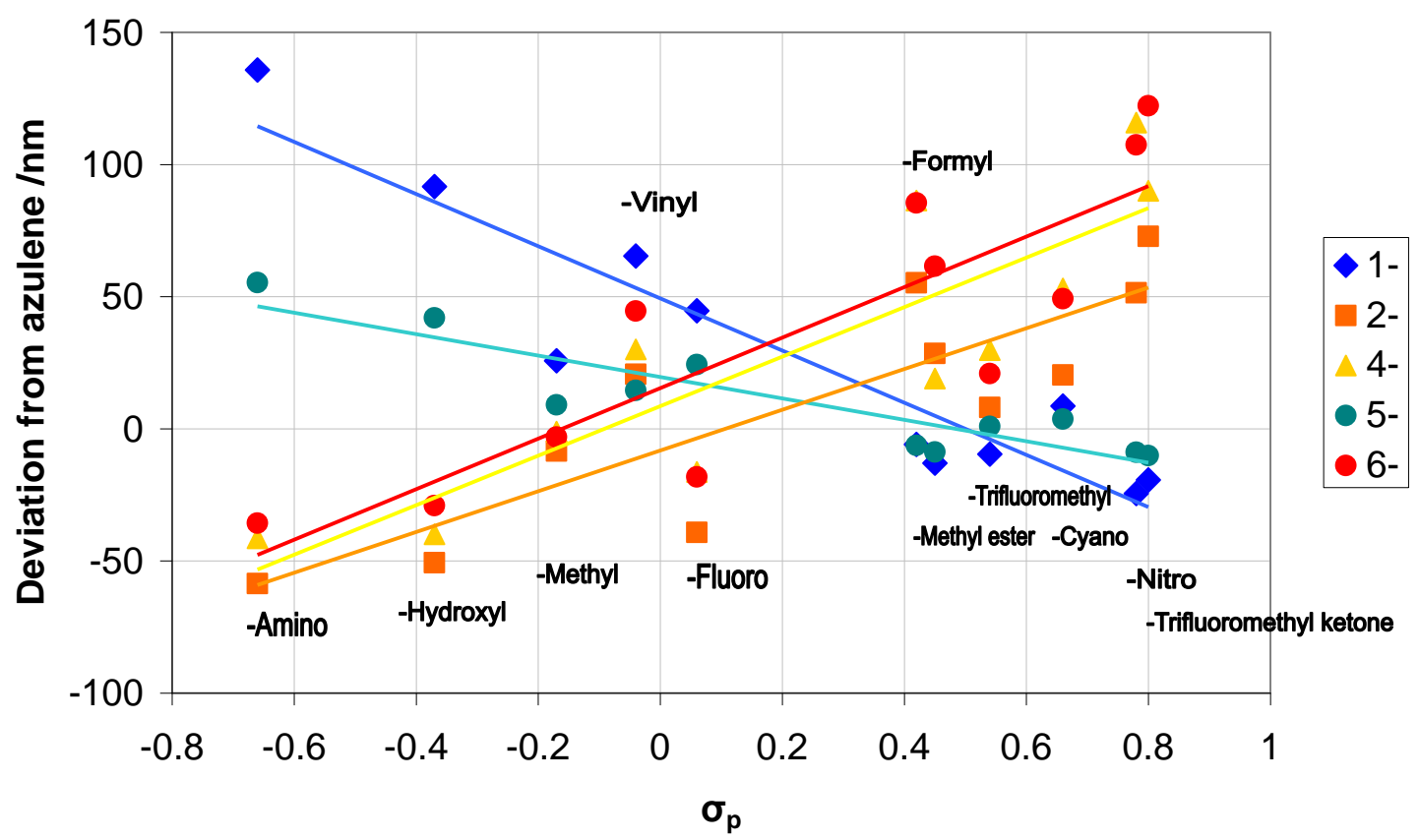

Figure 2.60 $\sigma_{\mathrm{p}}$ vs. absorption wavelength for a series of derivatives at positions 1-6

The overall trend in Figure 2.60 can be seen clearly. The absorption wavelengths of 1- and 5-substituted azulenes become shorter as the substituent becomes more electron-withdrawing, with the effect being less pronounced on the 5-position than on the 1- position. Conversely, the absorption wavelengths of the 2-, 4- and 6- substituted azulenes lengthen with increasing substituent electron-withdrawing ability.

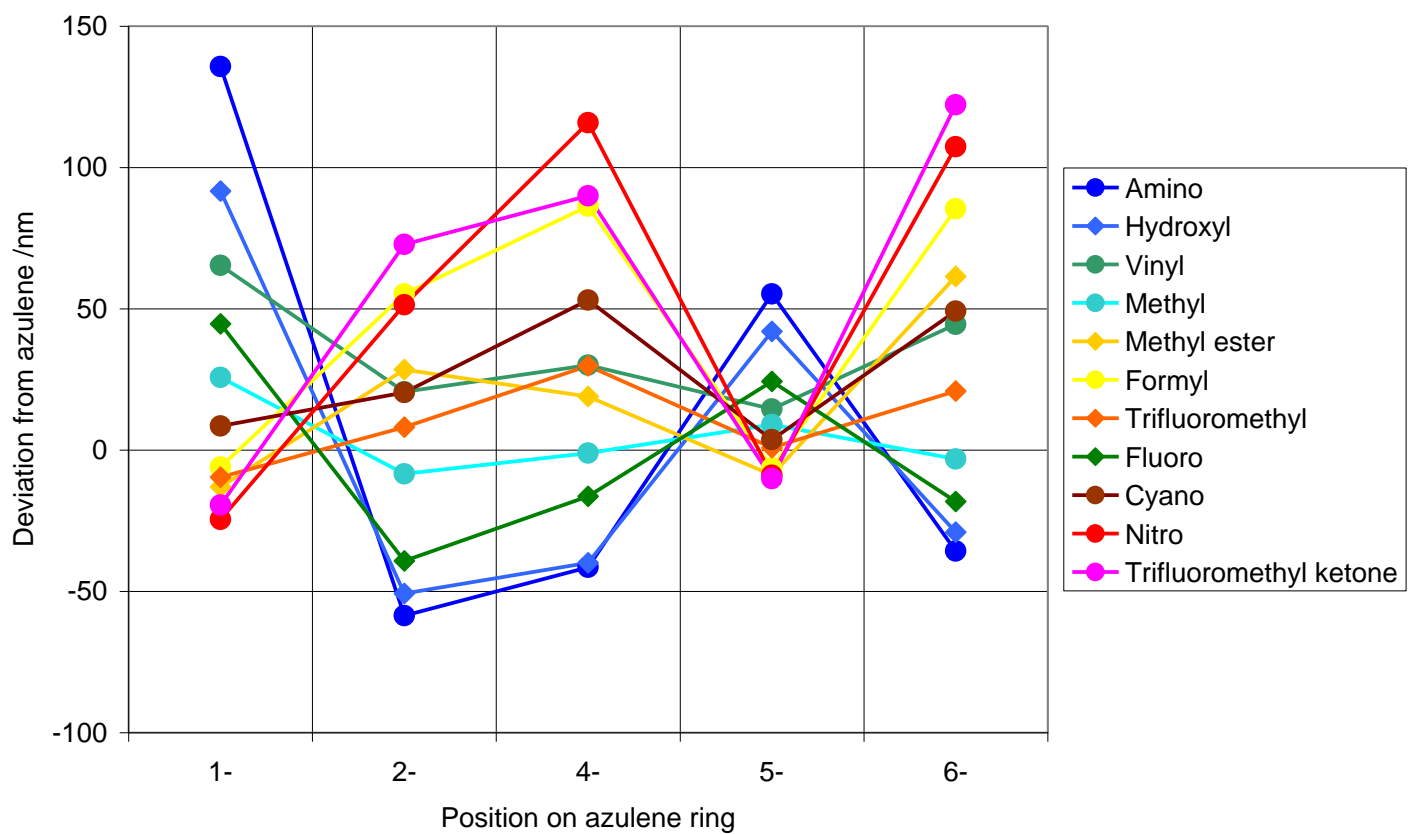

Figure 2.61 Absorption wavelength vs position on azulene ring for all derivatives 
There are some notable exceptions to this trend. The vinylazulenes were all calculated to have longer absorption wavelength than azulene itself. This can be rationalised by the effect of extension of the conjugation dominating any small resonance or inductive effects. More puzzling, however are the deviations seen in the fluoro-, trifluoromethyl- and cyanoazulenes. The fluoro group appears to donate electrondensity to a surprisingly large extent, whereas the aggressively electron-withdrawing trifluoromethyl and cyano groups influence the absorption surprisingly little. The reason for this is unclear, although it is suspected that $\pi$-conjugation between the substituent and the azulene ring is necessary in order for the electronic structure to be influenced considerably.

This hypothesis is given credence by the experimental observation that the ester $\mathbf{3 1}$ and the lactone 44 exhibit different colours. The ester appears blue-violet and the lactone appears magenta, which when considering the rules outlined in section 1.2.3 would suggest the carbonyl in the lactone was somehow more electron withdrawing than in the ester.
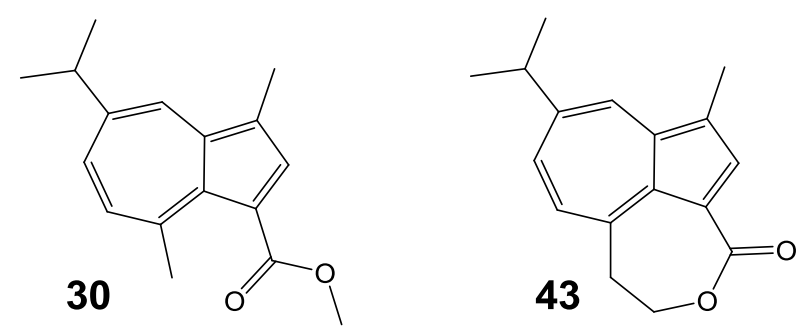

Figure 2.62 The ester 30 and the lactone $\mathbf{4 3}$ exhibit different colours despite their chemical similarities However this difference can be explained by the fact that while free rotation - and hence an out-of-plane conformation - is possible between the guaiazulene moiety and the ester, the lactone carbonyl is essentially locked in place. Therefore p-overlap between the carbonyl and the guaiazulene moiety would be greater in the lactone than in the ester, resulting in a correspondingly larger electron-withdrawing effect on the electronic structure. While this gives evidence to the above hypothesis, a counterexample can be found in the small deviations of the calculated values for the $\pi$-conjugated cyano derivatives.

In summary, the validity of the model described by Table 1.1 in section 1.2.3 wherein the colour of an azulene derivative is dependant both on the position and electrondonating/withdrawing nature of the substituent was shown. This model appears to fit 
best with conjugated substituents such as aldehydes and carboxylates, but can be tentatively used for most functional groups as a rule of thumb.

\subsubsection{Calculation of the relative energies of the keto- and enol- forms of the aldehyde 63}

Due to the ongoing difficulty in synthesising the aldehyde 63, the relative energies of the keto and enol forms were explored computationally in order to ascertain whether the enol form plays a part in the reactivity of $\mathbf{6 3}$.

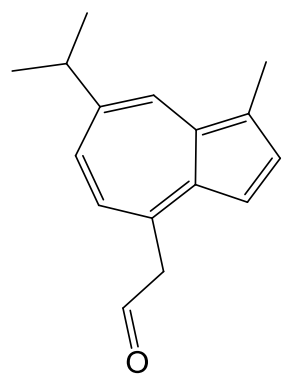

keto-

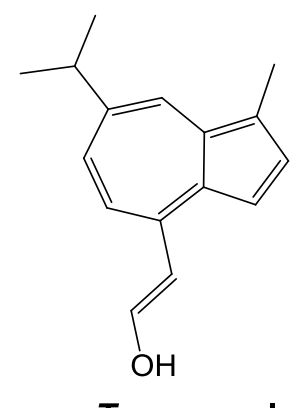

Trans-enol<smiles>Cc1ccc2c(/C=C\O)ccc(C(C)C)cc1-2</smiles>

Cis-enol

Figure 2.63 The keto- and enol- tautomers of the aldehyde $\mathbf{6 3}$

Geometry optimisations and frequency calculations were performed on these three tautomers using various exchange potentials and basis sets. Calculations were performed both in vacuo and with the SM 5.4 aqueous solvent approximation. One calculation was carried out using the SM 8 solvent approximation for dichloromethane. The relative energies of each tautomer are plotted in Table 2.3.

\begin{tabular}{|c|c|c|c|c|}
\hline \multirow[t]{2}{*}{ Level of theory } & \multirow[t]{2}{*}{ Solvent } & \multicolumn{3}{|c|}{ Relative energy $/ \mathrm{kJ} \mathrm{mol}^{-1}$} \\
\hline & & Keto & Cis-enol & Trans-enol \\
\hline \multirow[t]{3}{*}{ EDF2/6-31+G* } & In Vacuo & 0 & 55.2 & 65.9 \\
\hline & Aqueous & 0 & 54.9 & 54.0 \\
\hline & $\mathrm{CH}_{2} \mathrm{Cl}_{2}$ & 0 & 99.5 & 73.7 \\
\hline \multirow[t]{2}{*}{ EDF2/6-31G** } & In Vacuo & 0 & 44.2 & 54.4 \\
\hline & Aqueous & 0 & 44.0 & 42.6 \\
\hline \multirow[t]{2}{*}{ B3LYP/6-31G** } & In Vacuo & 0 & 42.5 & 54.1 \\
\hline & Aqueous & 0 & 41.9 & 42.0 \\
\hline
\end{tabular}

Table 2.3 Comparison of the relative energy of the three tautomers under different conditions and computational techniques

It was found that the keto tautomer was more stable than either enol tautomer by an average of $\sim 55 \mathrm{~kJ} \mathrm{~mol}^{-1}$. Calculations performed in vacuo predicted the cis enol to be more stable than the trans enol, whereas calculations performed with a solvent approximation either produced a negligible difference in energy between the two enol tautomers, or predicted the trans tautomer to be more stable. 


\subsection{Conclusions and future directions}

Throughout this research, many interesting results were obtained, some of which were unexpected and surprising. Although no usable novel protecting group system was discovered during this research, it is believed that the foundation has been laid down for the development of a novel chromophoric protecting group system in the near future.

As perhaps was inevitable with the open-ended nature of the project, there were many ideas and designs that were only briefly investigated beyond pen and paper, and many more that were never investigated at all. For instance, a late idea involved the use of guaiazulene sulfonamides as a chromophoric protecting group for amines. The use of sulfonamides as protecting groups for amines is widely practised, and a route to sulfonamides from guaiazulene using thionyl chloride and amines is known. ${ }^{48}$ Subsequent deprotection could be induced by addition of reagents such as samarium iodide or lithium naphthalide.

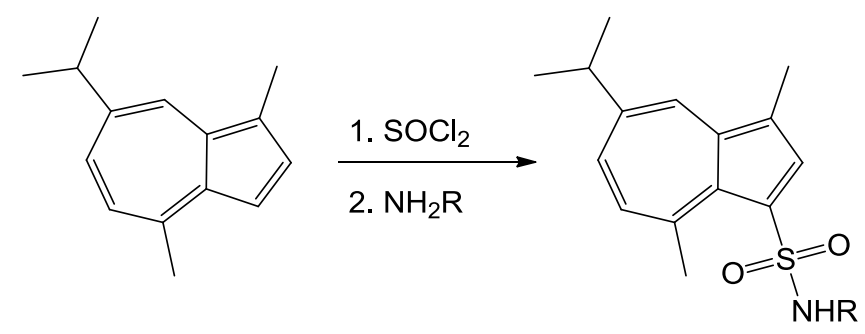

Figure 2.64 Synthesis of a guaiazulene sulfonamide

Many of the lines of research that had been investigated encountered problems of one kind or another. Each one could potentially have been overcome with more research, and it was often tricky to judge how much effort should be expended on any one area. The amine 22 showed promise as a protecting group, but requires further derivatisation in order to lower the tendency to react with itself intermolecularly. It is believed this can be achieved by addition of electron withdrawing groups or removal of the electron donating alkyl groups from the azulene moiety.

There are further ways in which the synthesis of aldehyde $\mathbf{6 3}$ may be achieved. For example, addition of a Weinreb amide to the guaiazulenide anion could conceivably generate the desired aldehyde, as could addition of glyoxal to the guaiazulenide anion followed by periodate cleavage. 


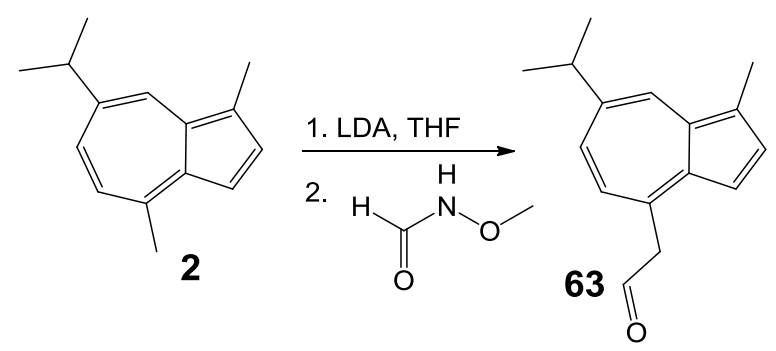

Figure 2.65 Possible synthetic route to 63 using a Weinreb amide

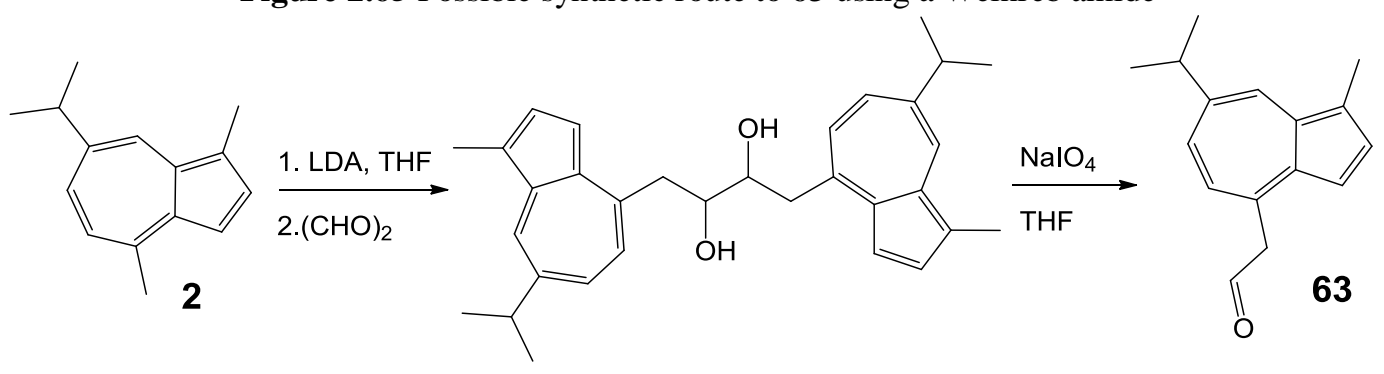

Figure 2.66 Possible two-step synthetic route to $\mathbf{6 3}$

Further investigation of the reactivity of silyl chlorides to the guaiazulenide ion is also necessary in order to explain the contradictory behaviour observed between chlorotrimethylsilane and dichlorodimethylsilane.

Azulene-functionalised PS-DVB resins also deserve more investigation. Evidence for their formation and subsequent reactions was obtained visually and through analysis of the mass balance. The performance of this guaiazulene-functionalised resin as a potential scavenger resin was demonstrated, if only qualitatively. The coupling of azulene onto resins other than Merrifield resin (such as Wang or Tentagel resin, with longer linkers between the polymer and the functional group) is needed for adequate characterisation. This was not done during the project due to the high cost of these resins.

Another possibility involving azulene-functionalised PS-DVP resins was as a stationary phase in reverse-phase chromatography. It was theorised the permanent dipole and polarisability of azulene could allow a functionalised stationary phase to exhibit unique retention of certain classes of compounds, enabling novel forms of chromatographic separation. In addition to this, some azulene derivatives exhibit changes in colour when exposed to various anions ${ }^{49,50}$ which opens up the possibility for the visual tracking through colour changes in the stationary phase of species that would otherwise be colourless. 
Finally, there are many outstanding questions of a theoretical nature about azulene derivatives. While the effects certain functional groups have on the azulene moiety are by now fairly well established through computational and experimental means, much less is known about the effect the azulene moiety itself has on these functional groups. For instance, it has been noted that the carbonyl stretches of 3-guaiazulene derivatives are anomalously low - the amide 32 exhibits a carbonyl stretch at 1624 $\mathrm{cm}^{-1}$, lower than that of urea. Coupled with the difficulty encountered with the synthesis of an acetal from the aldehyde 19, this suggests the 3- position of guaiazulene is highly electron donating. This suggests in turn that the presence of the azulene moiety may decrease the reactivity of these carbonyl groups, paving the way to graduated lability in systems with non-azulenic protecting groups also present.

Although there are many obstacles yet to be overcome, azulene derivatives show much promise as chromophoric and self-indicating tools in organic synthesis. 


\section{Chapter 3}

\section{Experimental}

\subsection{General procedures}

Unless otherwise stated, all reactions were carried out in flame-dried glass apparatus under an argon atmosphere. All reaction solvents were distilled prior to use. THF was distilled over benzophenone/sodium wire under argon, dichloromethane was distilled over $\mathrm{CaH}_{2}$ under argon and anhydrous DMF from a commercial Sigma-Aldrich dry bottle was used. Diisopropylamine, oxalyl chloride and phosphorous oxychloride were distilled from sodium wire under argon and stored in dry bottles before use. $n$ butyllithium was purchased from Sigma-Aldrich and used as received.

${ }^{1} \mathrm{H}$ and ${ }^{13} \mathrm{C}$ NMR spectra were obtained using a Varian Unity Inova $500^{\mathrm{TM}}$ spectrometer $\left(500 \mathrm{MHz}\right.$ for ${ }^{1} \mathrm{H}$ and $125 \mathrm{MHz}$ for $\left.{ }^{13} \mathrm{C}\right)$. Infra-red spectra were obtained using a Perkin-Elmer Spectrum One ${ }^{\mathrm{TM}}$ FTIR spectrometer. High resolution mass spectrometry was obtained using a Waters Q-TOF Premier ${ }^{\mathrm{TM}}$ Tandem Mass Spectrometer. UV-Vis spectra were obtained using a Varian Cary ${ }^{\mathrm{TM}} 100$ scan UVVIS Spectrophotometer. Melting points were determined with a Gallenkemp melting point apparatus.

\subsubsection{General procedure for LDA deprotonation reactions}

LDA was made up by adding approximately 1 to 1.1 equivalents of $2 \mathrm{M} \mathrm{n}$-BuLi in cyclohexane to 1 equivalent of dry diisopropylamine in approximately 9 equivalents of THF at $0{ }^{\circ} \mathrm{C}$ under argon in a flame-dried RBF with stirrer. The mixture would usually remain colourless but sometimes developed a brown colouration that did not affect the reactivity of the LDA. This mixture was added dropwise into the mixture containing the guaiazulene derivative and a colour change from blue to orange or brown was normally observed. Depending on ambient humidity, it could take from 1 equivalent to as many as 3 equivalents for this deprotonation to occur. This deprotonation was monitored visually and addition of LDA would stop as soon as the 
mixture ceases to noticeably change colour. A mixture containing fully deprotonated guaiazulene appears orange-red and is noticeably less opaque than the parent azulene.

\subsubsection{General procedure for purification}

All separations were monitored visually. Typically, all coloured products would dissolve predominantly in the organic phase. In situations where the coloured products were present in the aqueous phase, extraction with an appropriate organic solvent was normally repeated until no coloured compounds were visible in the aqueous phase. The number of extractions varies depending on the polarity of the coloured products, but this method is successful at dealing with both pure hydrocarbons and azulenic cations such as the iminium 19.

Unless otherwise stated, all flash chromatography was performed using silica as the static phase and a combination of hexanes, $\mathrm{CH}_{2} \mathrm{Cl}_{2}, \mathrm{Et}_{2} \mathrm{O}$ and $\mathrm{MeOH}$ as the mobile phase.

As with phase-separations, column chromatography was monitored visually. Except for the most challenging of separations, fractions were eschewed in favour of collecting the compounds directly into round-bottomed flasks as they eluted. In cases where streaking occurred or where several compounds eluted together, multiple samples were collected and analysed individually.

A green or brown colouration of the silica would almost always develop during column chromatography. This is the result of the acid-catalysed oxidative dimerisation of guaiazulene and its derivatives. To the author's knowledge, this cannot be avoided.

As the azulene chromophore is relatively strongly absorbing, sub-milligram quantities of various unidentified compounds were occasionally observed. These compounds almost never gave clean NMR spectra, often degraded quickly, and were often ignored. 


\subsubsection{Identification of compounds through the use of TLC}

Owing to the colour of azulene derivatives, many compounds could be tentatively identified from various traits during TLC. Most derivatives retain their colour while on the TLC plate, while a few, such as the aldehyde 19, would exhibit a colour change. Even when several compounds of the same colour are present on a TLC plate, there are certain features that may allow differentiation between them. For instance, guaiazulene itself runs at an $R_{\mathrm{f}}$ value of 0.5 in hexanes, whereas its alkyl derivatives would tend to both streak and run slightly more slowly. Upon staining of the TLC with either vanillin or anisaldehyde dip, colour changes could be observed even without charring. Guaiazulene and many of its blue derivatives would change colour to crimson, but at different rates with guaiazulene changing colour at the fastest rate. Considering most reactions that involve electrophilic attack on the guaiazulenide anion never fully go to completion, this trait is useful when separating guaiazulene from the guaiazulene derivatives.

\subsection{Experimental details}

\section{7-Isopropyl 1,4-dimethylazulene-3-carbaldehyde (19)}

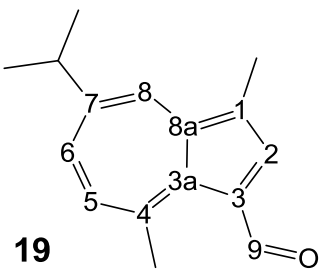

Adapted from a synthesis reported by K. Hafner et al., guaiazulene (198.9 mg, $0.988 \mathrm{mmol})$ in DMF (6 mL) was added to an RBF under standard conditions. Vilsmeier reagent was prepared by adding $\mathrm{POCl}_{3}(0.9 \mathrm{~mL}, 9.7 \mathrm{mmol})$ to $\mathrm{DMF}$ $(1.8 \mathrm{~mL}, 23 \mathrm{mmol})$ under inert atmosphere and $0.4 \mathrm{~mL}$ of this

solution was added to the reaction mixture. An instantaneous colour change from blue to green and then to red-orange was observed. A solution of $\mathrm{NaOH}(1.22 \mathrm{~g}, 30 \mathrm{mmol})$ in $\mathrm{H}_{2} \mathrm{O}(10 \mathrm{~mL})$ was added and the reaction left to stand for $10 \mathrm{~min}$. at RT. The brown mixture was extracted with $\mathrm{CH}_{2} \mathrm{Cl}_{2}$, followed by another extraction using $\mathrm{CuSO}_{4}(\mathrm{aq}) /$ hexanes in order to remove residual DMF. Flash chromatography was performed on the residue, eluting with $\mathrm{CH}_{2} \mathrm{Cl}_{2}$ to afford $174.8 \mathrm{mg}(77 \%)$ of the title compound as a green-brown solid that appears purple in solution.

${ }^{1} \mathrm{H}-\mathrm{NMR}:\left(\mathrm{CDCl}_{3}\right) \delta_{\mathrm{H}} 10.64$ (s, 1H, H-9), 8.29 (s, 1H, H-8), 8.23 (s, 1H, H-2), 7.59 (d, $\mathrm{J}=10 \mathrm{~Hz}, 1 \mathrm{H}, \mathrm{H}-6), 7.43$ (d, J=10 Hz, 1H, H-5), 3.12-3.20 (sep, J=7 Hz, 1H, ${ }^{\mathrm{i}} \operatorname{Pr}$ ), 3.15 
(s, 3H, 4-Me), 2.59 (s, 3H, 1-Me), 1.39 (d, J=7 Hz, 6H, $\left.{ }^{\mathrm{i}} \mathrm{Pr}\right) ;{ }^{13} \mathrm{C}-\mathrm{NMR}\left(\mathrm{CDCl}_{3}\right) \delta_{\mathrm{C}}$ 186.3 (CH, C-9), 147.0 (C, C-7), 146.6 (C, C-4), 143.5 (C, C-8a), 139.4 (CH, C-2), 139.2 (C, C-3a), 131.6 (CH, C-6), 135.4 (CH, C-8), 132.5 (CH, C-5), 127.4 (C, C-3), 127.2 (C, C-1), $38.2\left(\mathrm{CH},{ }^{\mathrm{i}} \mathrm{Pr}\right), 30.15\left(\mathrm{CH}_{3}, 4-\mathrm{Me}\right), 24.6\left(\mathrm{CH}_{3},{ }^{\mathrm{i}} \mathrm{Pr}\right), 13.0\left(\mathrm{CH}_{3}, 1-\mathrm{Me}\right)$; $\mathrm{UV}-\mathrm{V}$ is $\left(\mathrm{CH}_{2} \mathrm{Cl}_{2}\right) \lambda_{\max }: 553 \mathrm{~nm}$.

Methyl -1-((7-isopropyl-1,4-dimethylazulen-3-yl )-2-oxo ethanoate (30)

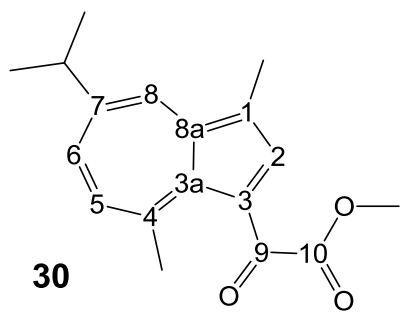

Guaiazulene (10.4 mg, $0.052 \mathrm{mmol})$ in dichloromethane (2 $\mathrm{mL})$ was added to an RBF under standard conditions. Oxalyl chloride $(5 \mu \mathrm{L}, 0.057 \mathrm{mmol})$ was added, and a colour change from blue to orange observed. After 1 minute, $\mathrm{MeOH}$ (1 mL, $25 \mathrm{mmol}$ ) was added, followed by

pyridine $(1.1 \mathrm{~mL})$ after 15 minutes. A separation was performed using $\mathrm{H}_{2} \mathrm{O} / \mathrm{CH}_{2} \mathrm{Cl}_{2}$ and the resultant organic fraction purified by column chromatography (gradient of hexanes $\rightarrow \mathrm{CH}_{2} \mathrm{Cl}_{2} \rightarrow \mathrm{MeOH}$ ) to afford $37 \mathrm{mg}$ of the title compound as an orange-red compound adsorbed onto silica.

${ }^{1} \mathrm{H}-\mathrm{NMR}:\left(\mathrm{CDCl}_{3}\right) \delta_{\mathrm{H}} 8.28(\mathrm{~d}, \mathrm{~J}=2 \mathrm{~Hz}, 1 \mathrm{H}, \mathrm{H}-8), 8.00$ (s, 1H, H-2), 7.67 (dd, J=11 Hz, 2 Hz, 1H, H-6), 7.49 (d, J=11 Hz, 1H, H-5), 3.98 (s, 3H, OMe), 3.21-3.12 (sep, J=7 $\mathrm{Hz}, 1 \mathrm{H},{ }^{\mathrm{i}} \mathrm{Pr}$ ), 3.00 (s, 3H, 4-Me), 2.56 (s, 3H, 1-Me), 1.39 (d, J=7 Hz, 6H, ${ }^{\mathrm{i}} \mathrm{Pr}$ ); ${ }^{13}$ C-NMR: $\left(\mathrm{CDCl}_{3}\right) \delta_{\mathrm{C}} 179.8$ (C, C-9), 166.0 (C, C-10), 150.4 (C, C-4), 147.7 (C, C7), 144.1 (C, C-8a), 142.9 (CH, C-2), 139.9 (C, C-3a), 137.0 (CH, C-6), 134.7 (CH, C-8), 134.2 (CH, C-5), 125.5 (C, C-1), 121.0 (C, C-3), $52.7\left(\mathrm{CH}_{3}, \mathrm{OMe}\right), 38.2(\mathrm{CH}$, $\left.{ }^{\mathrm{i}} \mathrm{Pr}\right), 28.9\left(\mathrm{CH}_{3}, 4-\mathrm{Me}\right), 24.5\left(\mathrm{CH}_{3},{ }^{\mathrm{i}} \mathrm{Pr}\right), 12.9\left(\mathrm{CH}_{3}, 1-\mathrm{Me}\right)$.

\section{7-Isopropyl-1,4-dimethylazulene-3-methyl ester (31)}

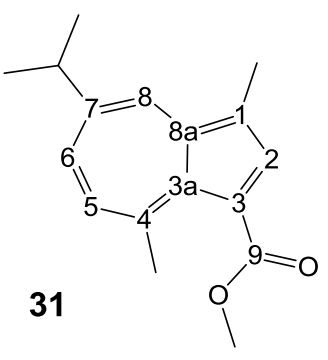

Guaiazulene (115 mg, $0.580 \mathrm{mmol})$ in $\mathrm{CH}_{2} \mathrm{Cl}_{2}(10 \mathrm{~mL})$ was added to an RBF under standard conditions. $(\mathrm{COCl})_{2}(0.1 \mathrm{~mL}$, $1.2 \mathrm{mmol}$ ) was added dropwise, at which point an instantaneous colour change from blue to orange-red was observed. After $1 \mathrm{hr}$,

$\mathrm{MeOH}(0.1 \mathrm{~mL}, 2.5 \mathrm{mmol})$ was added dropwise. $\mathrm{H}_{2} \mathrm{O}$ was then added and organic material was extracted using $\mathrm{CH}_{2} \mathrm{Cl}_{2}$.

Column chromatography was performed in $\mathrm{CH}_{2} \mathrm{Cl}_{2}$, and afforded $133 \mathrm{mg}$ (90\%) of the title compound as an indigo oil. 
${ }^{1} \mathrm{H}-\mathrm{NMR}:\left(\mathrm{CDCl}_{3}\right) \delta_{\mathrm{H}} 8.26(\mathrm{~d}, \mathrm{~J}=2 \mathrm{~Hz}, 1 \mathrm{H}, \mathrm{H}-8), 7.96$ (s, 1H, H-2), 7.54 (dd, J=11 Hz, $2 \mathrm{~Hz}, 1 \mathrm{H}, \mathrm{H}-6$ ), 7.28 (d, J=11 Hz, 1H, H-5), 3.93 (s, 3H, OMe), 3.17-3.09 (sep, J=7 $\left.\mathrm{Hz}, 1 \mathrm{H},{ }^{\mathrm{i}} \mathrm{Pr}\right), 2.98$ (s, 3H, 4-Me), 2.61 (s, 3H, 1-Me), 1.39 (d, J=7 Hz, 6H, $\left.{ }^{\mathrm{i}} \mathrm{Pr}\right),{ }^{13} \mathrm{C}-$ NMR $\left(\mathrm{CDCl}_{3}\right) \delta_{\mathrm{C}} 167.5$ (C, C-9), 147.3 (C, C-4), 143.6 (C, C-7), 140.4 (C, 8a), 140.3 (CH, C-2), 136.2 (C, C-3a), 135.9 (CH, C-6), 134.4 (CH, C-8), 130.7 (CH, C-5), 124.1 (C, C-1), 116.6 (C, C-3), $51.7\left(\mathrm{CH}_{3}, \mathrm{OMe}\right), 38.0\left(\mathrm{CH},{ }^{\mathrm{i}} \mathrm{Pr}\right), 27.8\left(\mathrm{CH}_{3}, 4-\mathrm{Me}\right)$, $24.6\left(\mathrm{CH}_{3},{ }^{\mathrm{i}} \mathrm{Pr}\right), 12.8\left(\mathrm{CH}_{3}, 1-\mathrm{Me}\right)$; IR (KBr): $v_{\max } 2924,2947,2852,1645,1435$, 1192, $1035 \mathrm{~cm}^{-1}$; HRMS: $m / z \mathrm{C}_{17} \mathrm{H}_{20} \mathrm{O}_{2} \mathrm{Na}^{+}[\mathrm{M}+\mathrm{Na}]^{+}$calcd 279.1361, found 279.1367 .

\section{$\mathbf{N}, \boldsymbol{N}$-Diethyl-7-isopropyl-1,4-dimethylazulen-3-amide (32)}

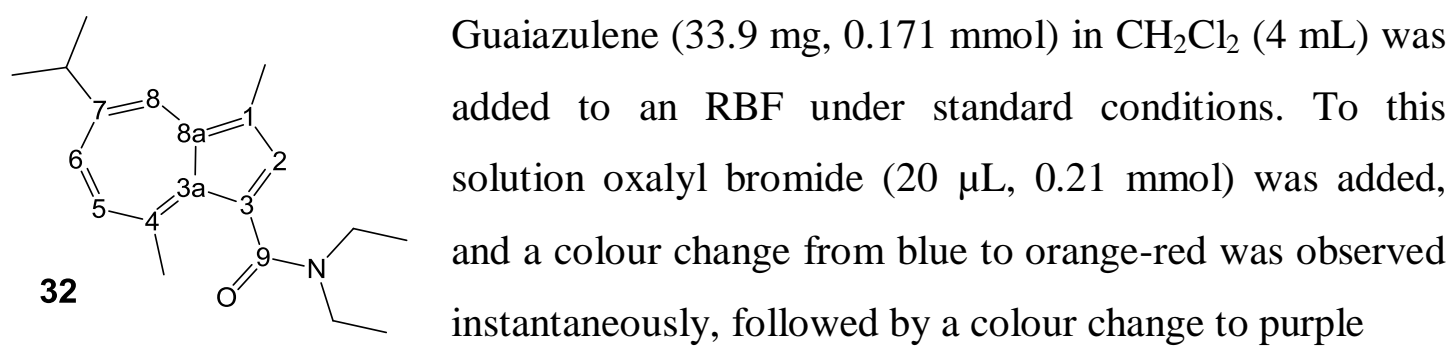

over the course of several minutes. After 20 minutes, $\mathrm{NHEt}_{2}(0.1 \mathrm{~mL}, 0.97 \mathrm{mmol})$ was added. A separation was performed on this mixture using $\mathrm{CH}_{2} \mathrm{Cl}_{2} / \mathrm{H}_{2} \mathrm{O}$, and the organic fraction purified by column chromatography (gradient of $\mathrm{CH}_{2} \mathrm{Cl}_{2} \rightarrow \mathrm{Et}_{2} \mathrm{O}$ ) to afford $10 \mathrm{mg}(20 \%)$ of the title compound as a blue oil.

${ }^{1} \mathrm{H}-\mathrm{NMR}:\left(\mathrm{CDCl}_{3}\right) \delta_{\mathrm{H}} 8.18$ (d, J=2 Hz, 1H, H-8), 7.50 (s, 1H, H-2), 7.41 (dd, J=11 Hz, $2 \mathrm{~Hz}, 1 \mathrm{H}, \mathrm{H}-6), 7.01$ (d, J=11 Hz, 1H, H-5), 3.91 (m, 1H, Et), 3.39 (m, 1H, Et), 3.22 (m, 1H, Et), 3.15 (m, 1H, Et), 3.08 (sep, J=7 Hz, 1H, ${ }^{\mathrm{i}} \mathrm{Pr}$ ), 2.79 (s, 3H, 4-Me), 2.62 (s, 3H, 1-Me), 1.36 (d, J=7 Hz, 6H, ${ }^{\mathrm{i}} \mathrm{Pr}$ ), 1.29 (t, J=7 Hz, 3H, Et), 1.02 (t, J=7 Hz, 3H, $\mathrm{Et}) ;{ }^{13} \mathrm{C}-\mathrm{NMR}\left(\mathrm{CDCl}_{3}\right) \delta_{\mathrm{C}} 171.6(\mathrm{C}, \mathrm{C}-9), 145.4$ (C, C-4). 140.7 (C, C-7), $137.2(\mathrm{C}$, C-8a), 135.5 (CH, C-2), 135.4 (CH, C-6), 134.5 (CH, C-8), 132.0 (C, C-3a), 127.1 (CH, C-5), 124.6 (C, C-1), 122.3 (C, C-3), $43.2\left(\mathrm{CH}_{2}, \mathrm{Et}\right), 38.8\left(\mathrm{CH}_{2}, \mathrm{Et}\right), 38.0(\mathrm{CH}$, $\left.{ }^{\mathrm{i}} \mathrm{Pr}\right), 25.2\left(\mathrm{CH}_{3}, 4-\mathrm{Me}\right), 24.7\left(\mathrm{CH}_{3},{ }^{\mathrm{i}} \mathrm{Pr}\right), 13.8\left(\mathrm{CH}_{3}, 1-\mathrm{Me}\right), 12.9\left(\mathrm{CH}_{3}, \mathrm{Et}\right), 12.4\left(\mathrm{CH}_{3}\right.$, $\mathrm{Et})$; UV-Vis $\left(\mathrm{CH}_{2} \mathrm{Cl}_{2}\right) \lambda_{\max }: 599 \mathrm{~nm}$; IR (KBr): $v_{\max } 2965,1624,1439,1266,644 \mathrm{~cm}^{-1}$; HRMS: $m / z \mathrm{C}_{20} \mathrm{H}_{28} \mathrm{NO}^{+}[\mathrm{M}+\mathrm{H}]^{+}$calcd 298.2171, found 298.2170. 


\section{1,4-Dimethyl-7-isopropylazulene-3-tert-butyl ester (47)}

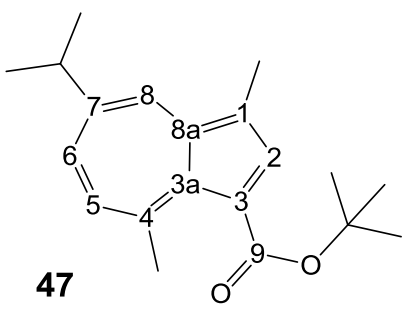

Guaiazulene (209.6 mg, $1.057 \mathrm{mmol})$ in $\mathrm{CH}_{2} \mathrm{Cl}_{2}(10 \mathrm{~mL})$ was added to an RBF under standard conditions. Oxalyl chloride $(0.1 \mathrm{~mL}, 1.2 \mathrm{mmol})$ was added dropwise to this mixture and a colour change to orange was observed. After 40 minutes, tert-butyl alcohol $(0.3 \mathrm{~mL}, 3.2 \mathrm{mmol})$ was

added, followed 5 minutes later by pyridine $(0.4 \mathrm{~mL}, 5.0 \mathrm{mmol})$ upon addition of which caused a violent evolution of gas. A separation was performed using $\mathrm{H}_{2} \mathrm{O} / \mathrm{CH}_{2} \mathrm{Cl}_{2}$, followed by column chromatography (gradient of hexanes $\rightarrow \mathrm{CH}_{2} \mathrm{Cl}_{2} \rightarrow \mathrm{Et}_{2} \mathrm{O}$ ) to afford $216 \mathrm{mg}(72 \%)$ of the title compound as an indigo oil.

${ }^{1} \mathrm{H}-\mathrm{NMR}:\left(\mathrm{CDCl}_{3}\right) \delta_{\mathrm{H}} 8.21(\mathrm{~d}, \mathrm{~J}=2 \mathrm{~Hz} 1 \mathrm{H}, \mathrm{H}-8), 7.85$ (s, 1H, H-2), 7.49 (dd, J=11 Hz, 2 Hz, 1H, H-6), 7.21 (d, J=11 Hz, 1H, H-5) 3.10 (sep, J=7 Hz, 1H, ${ }^{\mathrm{i}} \operatorname{Pr}$ ), 2.96 (s, 3H, 4$\mathrm{Me}$ ), 2.59 (s, 3H, 1-Me), 1.63 (s, 9H, $\left.{ }^{\mathrm{t}} \mathrm{Bu}\right), 1.36$ (d, J=7 Hz, 6H, $\left.{ }^{\mathrm{i}} \mathrm{Pr}\right) ;{ }^{13} \mathrm{C}-\mathrm{NMR}$ $\left(\mathrm{CDCl}_{3}\right) \delta_{\mathrm{C}} 167.2(\mathrm{C}, \mathrm{C}-9), 146.9(\mathrm{C}, \mathrm{C}-4), 142.9(\mathrm{C}, \mathrm{C}-7), 140.0(\mathrm{CH}, \mathrm{C}-2), 139.8(\mathrm{C}$, C-8a), 135.6 (CH, C-6), 135.1 (C, C-3a), 134.3 (CH, C-8), 129.9 (CH, C-5), 123.8 (C, $\mathrm{C}-1), 119.4(\mathrm{C}, \mathrm{C}-3), 80.1$ (C, $\left.{ }^{\mathrm{t}} \mathrm{Bu}\right), 38.0\left(\mathrm{CH},{ }^{\mathrm{i}} \mathrm{Pr}\right), 28.4\left(\mathrm{CH}_{3},{ }^{\mathrm{t}} \mathrm{Bu}\right), 27.7\left(\mathrm{CH}_{3}, 4-\mathrm{Me}\right)$, $24.6\left(\mathrm{CH}_{3},{ }^{\mathrm{i}} \mathrm{Pr}\right), 12.8\left(\mathrm{CH}_{3}, 1-\mathrm{Me}\right)$

N-cyclohexyl-1,4-dimethyl-7-isopropylazulene-3-methylenimine (20) and $\mathbf{N}, \mathbf{N}$-cyclohexyl-[1,4-dimethyl-7-isopropylazulen-3yl]methanamine (22)

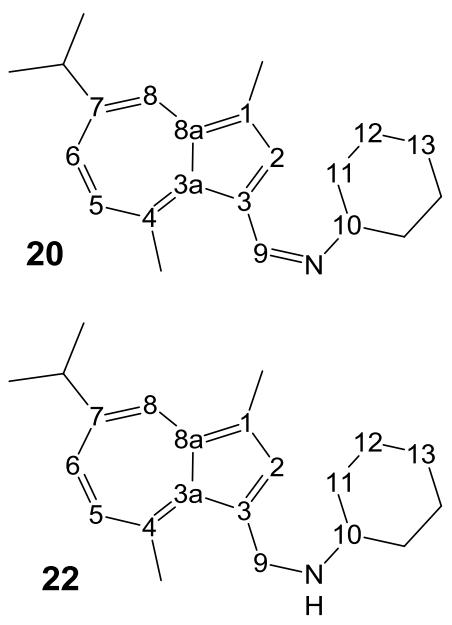

Guaiazulene (23.1 mg, $0.116 \mathrm{mmol})$ in DMF ( $3 \mathrm{~mL}$ ) was added to an RBF under standard conditions. Vilsmeier reagent was prepared under standard conditions $\left(0.14 \mathrm{~mL} \mathrm{POCl}_{3}\right.$ in $\left.1.2 \mathrm{~mL} \mathrm{DMF}\right)$ and $0.14 \mathrm{~mL}$ of this solution was added to the reaction mixture. A colour change from blue to orange-red was observed. After 5 minutes, cyclohexylamine $(1.0 \mathrm{~mL}, 8.7 \mathrm{mmol})$ was added to the reaction mixture and a colour change to teal was noted. A small quantity of this reaction mixture was removed and triturated with hexanes to obtain $\mathbf{2 0}$ as a teal oil. $\mathrm{NaBH}_{4}(39 \mathrm{mg}, 1.0$ mmol) was added to the reaction mixture and a subtle colour change to blue-green was observed. A separation was performed using $\mathrm{CH}_{2} \mathrm{Cl}_{2} / \mathrm{H}_{2} \mathrm{O}$ and the organic fraction was reduced under rotary evaporation and purified by column chromatography to afford $22 \mathrm{mg}(63 \%)$ of $\mathbf{2 2}$ as a blue-green oil. 
(20): ${ }^{1} \mathrm{H}-\mathrm{NMR}:\left(\mathrm{CDCl}_{3}\right) \delta_{\mathrm{H}} 9.15$ (s, 1H, H-9), 8.19 (s, 1H, H-2), 8.11 (s, 1H, H-8), 7.33 (d, J=7 Hz, 1H, H-6), 7.04 (d, J=11 Hz, 1H, H-5), 3.21-3.13 (m, 3H, H-10), 3.083.03 (sep, J=7 Hz, 1H, $\operatorname{Pr}$ ), 2.99 (s, 3H, 4-Me), 2.58 (s, 3H, 1-Me), 1.91-1.82 (complex m, Cy), 1.82-1.76 (complex m, Cy), 1.47-1.47 (complex m, Cy), 1.32-1.18, (complex m, Cy), 1.35 (d, J=7 Hz, 6H, $\left.{ }^{\mathrm{i}} \mathrm{Pr}\right) ;{ }^{13} \mathrm{C}-\mathrm{NMR}\left(\mathrm{CDCl}_{3}\right) \delta_{\mathrm{C}} 156.6(\mathrm{C}, \mathrm{C}-3 \mathrm{a})$, 156.4 (CH, C-9), 145.0 (C, C-4), 142.5 (C, C-7), 141.2 (C, C-8a), 138.1 (CH, C-2), 134.7 (CH, C-6), 134.0 (CH, C-8), 128.5 (CH, C-5), 126.2 (C, C-1), 125.4 (C, C-3), $70.6(\mathrm{CH}, \mathrm{C}-10), 37.9\left(\mathrm{CH},{ }^{\mathrm{i}} \mathrm{Pr}\right), 34.8\left(\mathrm{CH}_{2}, \mathrm{Cy}\right), 33.6\left(\mathrm{CH}_{2}, \mathrm{Cy}\right), 29.4\left(\mathrm{CH}_{3}, 4-\mathrm{Me}\right)$, 25.7-24.6 ( $\left.\mathrm{CH}_{2}, \mathrm{Cy}\right), 24.6\left(\mathrm{CH}_{3},{ }^{\mathrm{i}} \mathrm{Pr}\right), 12.8\left(\mathrm{CH}_{3}, 1-\mathrm{Me}\right)$.

(22): ${ }^{1} \mathrm{H}-\mathrm{NMR}:\left(\mathrm{CDCl}_{3}\right) \delta_{\mathrm{H}} 8.09$ (s, 1H, H-8), $7.61(\mathrm{~s}, 1 \mathrm{H}, \mathrm{H}-2), 7.30(\mathrm{~d}, \mathrm{~J}=11 \mathrm{~Hz}, 1 \mathrm{H}$, H-6), 6.92 (d, J=11 Hz, 1H, H-5) 4.230 (s, 2H, H-9), 3.08 (s, 3H, 4-Me), 3.06-2.99 (sep, J=7 Hz, 1H, ${ }^{\mathrm{i}} \mathrm{Pr}$ ), 2.68-2.62 (m, 1H, H-10), 2.61 (s, 3H, 1-Me), 1.98 (d, J=12 Hz, 2H, H-11), 1.75 (d, J=13 Hz, 2H, H-12), 1.63 (d, J=12 Hz, 1H, H-13), 1.34 (d, J=7 $\mathrm{Hz}, 6 \mathrm{H},{ }^{\mathrm{i}} \mathrm{Pr}$ ), 1.32-1.12 (complex m, J=12 Hz, 5H, H-11, 12, 13); ${ }^{13} \mathrm{C}-\mathrm{NMR}\left(\mathrm{CDCl}_{3}\right)$ $\delta_{\mathrm{C}} 145.7$ (C, C-4), 140.2 (CH, C-2), 139.2 (C, C-7), 137.8 (C, C-8a), 134.8 (CH, C-6), 133.4 (CH, C-8), 133.0 (C, C-3a), 126.9 (CH, C-5), 125.9 (C, C-3), 124.0 (C, C-1), $57.2(\mathrm{CH}, \mathrm{C}-10), 47.0\left(\mathrm{CH}_{2}, \mathrm{C}-9\right), 37.7\left(\mathrm{CH},{ }^{\mathrm{i}} \mathrm{Pr}\right), 33.4\left(\mathrm{CH}_{2}, \mathrm{C}-11\right), 26.3\left(\mathrm{CH}_{3}, 4-\right.$ $\mathrm{Me}), 26.2\left(\mathrm{CH}_{2}, \mathrm{C}-13\right), 25.1\left(\mathrm{CH}_{2}, \mathrm{C}-12\right), 24.7\left(\mathrm{CH}_{3},{ }^{\mathrm{i}} \mathrm{Pr}\right), 12.9\left(\mathrm{CH}_{3}, 1-\mathrm{Me}\right)$

\section{7-Isopropyl-1,3,4-trimethylazulene (29)}

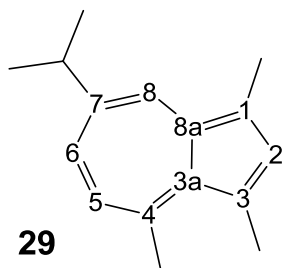

Aldehyde 19 (140.3 mg, $0.620 \mathrm{mmol})$ in THF (6 mL) was added to an RBF under standard conditions. A large excess of $\mathrm{NaBH}_{4}$ was added, followed by addition of freshly-distilled $\mathrm{BF}_{3} \cdot \mathrm{Et}_{2} \mathrm{O}$ $(0.1 \mathrm{~mL}, 0.81 \mathrm{mmol})$. An initial colour change from purple to yellow was observed, followed by a slower colour change to blue. A separation was performed using $\mathrm{CH}_{2} \mathrm{Cl}_{2} / \mathrm{H}_{2} \mathrm{O}$, and the organic fraction was reduced by rotary evaporation and purified by column chromatography (gradient of hexanes $\rightarrow \mathrm{CH}_{2} \mathrm{Cl}_{2}$ ) to afford $104 \mathrm{mg}$ (78\% yield) of the title compound as a blue-green oil.

${ }^{1} \mathrm{H}-\mathrm{NMR}:\left(\mathrm{CDCl}_{3}\right) \delta_{\mathrm{H}} 8.06$ (d, J=2 Hz, 1H, H-8), 7.45 (s, 1H, H-2), 7.25 (dd, J=11 Hz, 2 Hz, 1H, H-6), 6.80 (d, J=11 Hz, 1H, H-5), 3.03 (sep, J=7 Hz, 1H, ${ }^{\mathrm{i}} \operatorname{Pr}$ ), 3.01 (s, 3H, 4-Me), 2.90 (s, 3H, 3-Me), 2.64 (s, 3H, 1-Me), 1.37 (d, J=7 Hz, 6H, ${ }^{\mathrm{i}} \mathrm{Pr}$ ); ${ }^{13} \mathrm{C}-\mathrm{NMR}$ $\left(\mathrm{CDCl}_{3}\right) \delta_{\mathrm{C}} 145.9(\mathrm{C}, \mathrm{C}-4), 140.7(\mathrm{CH}, \mathrm{C}-2), 138.2$ (C, C-7), 137.3 (C, C-8a), 134.9 (CH, C-6), 133.3 (CH, C-8), 133.1 (C, C-3a), 125.6 (CH, C-5), 123.7 (C, C-1), 123.6 
(C, C-3), $37.7\left(\mathrm{CH},{ }^{\mathrm{i}} \mathrm{Pr}\right), 26.8\left(\mathrm{CH}_{3}, 4-\mathrm{Me}\right), 24.7\left(\mathrm{CH}_{3},{ }^{\mathrm{i}} \mathrm{Pr}\right), 18.7\left(\mathrm{CH}_{3}, 3-\mathrm{Me}\right), 12.8$ $\left(\mathrm{CH}_{3}, 1-\mathrm{Me}\right)$

\section{4-(2-Phenylethyl)-7-isopropyl-1-methylazulene (37)}

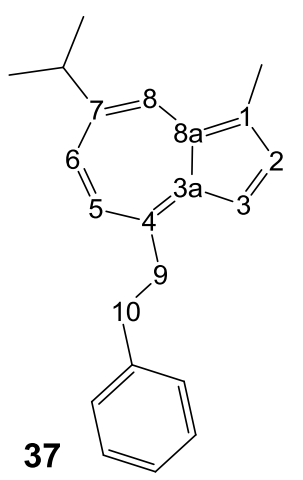

Guaiazulene $(58.7 \mathrm{mg}, 0.296 \mathrm{mmol})$ in THF (4 mL) was added to an RBF under standard conditions. LDA was prepared under standard conditions ( $0.08 \mathrm{~mL}$ diisopropylamine, $2 \mathrm{~mL}$ THF and $0.3 \mathrm{~mL}$ of $2 \mathrm{M} n$-BuLi in cyclohexane). $1.1 \mathrm{~mL}$ of this mixture was added dropwise until a colour change to orange was observed. Benzyl chloride $(0.1 \mathrm{~mL}, 0.86 \mathrm{mmol})$ was added and reaction mixture changed colour back to blue over the course of

a minute. A separation was performed using $\mathrm{CH}_{2} \mathrm{Cl}_{2} / \mathrm{H}_{2} \mathrm{O}$ and the organic phase reduced under high vaccuum to remove excess benzyl chloride. Column chromatography (gradient of hexanes $\rightarrow \mathrm{CH}_{2} \mathrm{Cl}_{2}$ ) was performed, collecting the second blue fraction to obtain $69 \mathrm{mg}(82 \%)$ of the title compound as a blue oil.

${ }^{1} \mathrm{H}-\mathrm{NMR}:\left(\mathrm{CDCl}_{3}\right) \delta_{\mathrm{H}} 8.25$ (s, 1H, H-8), 7.71 (d, J=4 Hz, 1H, H-2), 7.47 (d, J=11 Hz, 1H, H-6), 7.41 (d, J=4 Hz, 1H, H-3), 7.38-7.33 (complex m, 3H, Ph), 7.30-7.26 (complex m, 2H, Ph), 7.06 (d, J=11 Hz, 1H, H-5), 3.48 (t, J=9 Hz, 2H, H-9), 3.17 (t, $J=8 \mathrm{~Hz}, 2 \mathrm{H}, \mathrm{H}-10), 3.12$ (sep, $J=7 \mathrm{~Hz}, 1 \mathrm{H},{ }^{\mathrm{i}} \mathrm{Pr}$ ), 2.72 (s, 3H, 1-Me), 1.41 (d, J=7 Hz, $\left.6 \mathrm{H},{ }^{\mathrm{i}} \mathrm{Pr}\right) ;{ }^{13} \mathrm{C}-\mathrm{NMR}\left(\mathrm{CDCl}_{3}\right) \delta_{\mathrm{C}} 148.1$ (C, C-4), 142.1 (C, Ph), 140.1 (C, C-7), 136.9 (C, C-3a), 136.5 (CH, C-2), 136.2 (C, C-8a), 135.3 (CH, C-6), 133.5 (CH, C-8), 128.5 (CH, Ph), 128.4 (CH, Ph), 125.3 (C, C-1), 124.7 (CH, C-5), 112.1 (CH, C-3), 40.6 $\left(\mathrm{CH}_{2}, \mathrm{C}-9\right), 38.3\left(\mathrm{CH},{ }^{\mathrm{i}} \mathrm{Pr}\right), 37.7\left(\mathrm{CH}_{2}, \mathrm{C}-10\right), 24.8\left(\mathrm{CH}_{3},{ }^{\mathrm{i}} \mathrm{Pr}\right), 13.0\left(\mathrm{CH}_{3}, 1-\mathrm{Me}\right)$; IR (KBr): $v_{\max } 3061,3025,2958,2861,1555,1461,1386,1025,920,698$; HRMS: $m / z$ $\mathrm{C}_{22} \mathrm{H}_{24} \mathrm{Na}^{+}[\mathrm{M}+\mathrm{Na}]^{+}$, calcd 311.1776, found 311.1774. 


\section{4-(2-Hydroxyethyl)-7-isopropyl-1-methylazulene (36)}

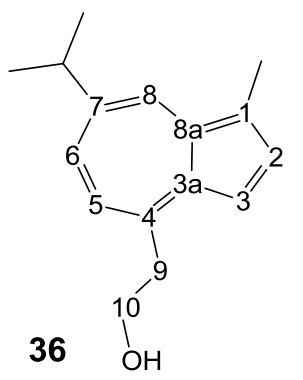

Guaiazulene (208.8 mg, $1.052 \mathrm{mmol})$ in THF $(9 \mathrm{~mL})$ was added to an RBF under standard conditions. LDA was made up under standard conditions ( $2 \mathrm{~mL}$ THF, $0.9 \mathrm{~mL}$ diisopropylamine and 3 $\mathrm{mL} 2 \mathrm{M} n$-BuLi in cyclohexane). $1.2 \mathrm{~mL}$ (1.16 eq.) of LDA was added to the reaction mixture, and reaction changed colour to orange. Formaldehyde (470 mg, $16 \mathrm{mmol}$ ) was added by

heating paraformaldehyde powder in a separate flask with a heatgun at $250{ }^{\circ} \mathrm{C}$, transferring the evolved formaldehyde vapour into the reaction mixture via cannula, and at this point, the reaction mixture changed colour to blue. The reaction was quenched with $\mathrm{H}_{2} \mathrm{O}$ and organic material was extracted using $\mathrm{CH}_{2} \mathrm{Cl}_{2}$ and purified by column chromatography (gradient of hexanes $\rightarrow \mathrm{CH}_{2} \mathrm{Cl}_{2}$ ), collecting the second blue fraction to obtain $161 \mathrm{mg}(67 \%)$ of the title compound as a viscous blue oil.

${ }^{1} \mathrm{H}-\mathrm{NMR}:\left(\mathrm{CDCl}_{3}\right) \delta_{\mathrm{H}} 8.22(\mathrm{~s}, 1 \mathrm{H}, \mathrm{H}-8), 7.68(\mathrm{~d}, \mathrm{~J}=4 \mathrm{~Hz}, 1 \mathrm{H}, \mathrm{H}-2), 7.45$ (d, J=11 Hz, 1H, H-6), 7.33 (d, J=4 Hz, 1H, H-3), 7.04 (d, J=10 Hz, 1H, H-5), 4.09 (td, J=6 Hz, 1 Hz, 2H, H-10), 3.42 (td, J=6 Hz, 1 Hz, 2H, H-9), 3.10 (sep, J=7 Hz, 1H, ${ }^{\mathrm{i}} \operatorname{Pr}$ ), 2.68 (s, $3 \mathrm{H}, 1-\mathrm{Me}), 1.39\left(\mathrm{dd}, \mathrm{J}=7 \mathrm{~Hz}, 1 \mathrm{~Hz}, 6 \mathrm{H},{ }^{\mathrm{i}} \mathrm{Pr}\right) ;{ }^{13} \mathrm{C}-\mathrm{NMR}\left(\mathrm{CDCl}_{3}\right) \delta_{\mathrm{C}} 144.6(\mathrm{C}, \mathrm{C}-4)$, 140.3 (C, C-7), 137.7 (C, C-3a), 136.7 (CH, C-2), 136.2 (CH, C-2), 135.2 (CH, C-6), 133.6 (CH, C-8), 125.7 (C, C-1), 124.8 (CH, C-5), 112.3 (CH, C-3), $63.7\left(\mathrm{CH}_{2}, \mathrm{C}-\right.$ 10), $41.4\left(\mathrm{CH}_{2}, \mathrm{C}-9\right), 38.3\left(\mathrm{CH},{ }^{\mathrm{i}} \mathrm{Pr}\right), 24.8\left(\mathrm{CH}_{3},{ }^{\mathrm{i}} \mathrm{Pr}\right), 13.0\left(\mathrm{CH}_{3}, 1-\mathrm{Me}\right)$.

\section{4-(2-Phenylethyl)-7-isopropyl-1-methylazulene-3-methyl ester (40) and} methyl 1-(4-(2-phenylethyl)-7-isopropyl-1-methylazulen-3-yl)-2-oxo ethanoate (41)
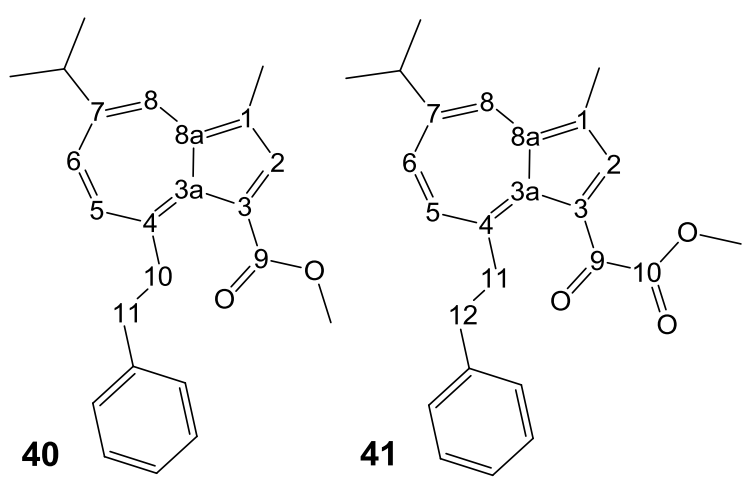

4-(2-Phenylethyl)-7-isopropyl-1methylazulene (43.7 $\mathrm{mg}, 0.191 \mathrm{mmol})$ in $\mathrm{CH}_{2} \mathrm{Cl}_{2}$ (4 mL) was added to an RBF under standard conditions. Oxalyl chloride (40 $\mu \mathrm{L}, 0.47 \mathrm{mmol})$ was added dropwise to reaction mixture, and a colour change to orange-red was

observed. After 1 minute, $\mathrm{MeOH}(0.8 \mathrm{~mL}, 19.7 \mathrm{mmol})$ was added, followed by pyridine $(0.5 \mathrm{~mL}, 6.206 \mathrm{mmol})$ after 5 minutes. A separation was performed using 
$\mathrm{H}_{2} \mathrm{O} / \mathrm{CH}_{2} \mathrm{Cl}_{2}$ and the organic fraction reduced under rotary evaporation and purified by column chromatography (gradient of hexanes $\rightarrow \mathrm{CH}_{2} \mathrm{Cl}_{2} \rightarrow \mathrm{Et}_{2} \mathrm{O}$ ) to obtain $14 \mathrm{mg}$ (27\%) of 4-(2-phenylethyl)-7-isopropyl-1-methylazulene-3-methyl ester as a highlyviscous purple oil and $28 \mathrm{mg}$ (49\%) of methyl 1-(4-(2-phenylethyl)-7-isopropyl-1methyl azulen-3-yl ) oxo ethanoate as an orange-red oil.

(40): ${ }^{1} \mathrm{H}-\mathrm{NMR}:\left(\mathrm{CDCl}_{3}\right) \delta_{\mathrm{H}} 8.27$ (s, 1H, H-8), 8.01 (s, 1H, H-2), 7.56 (d, J=11 Hz, 1H, H-6), 7.31 (d, J=11 Hz, 1H, H-5), 7.31-7.24 (m, J=7 Hz, 4H, Ph), 7.20 (t, J=7 Hz, 1H, Ph), 3.91 (s, 3H, OMe), 3.73 (t, J=9 Hz, 2H, H-10), 3.13 (sep, J=7 Hz, 1H, ${ }^{\mathrm{i}} \operatorname{Pr}$ ), 3.04 (t, J=8 Hz, 1H, H-11), 2.62 (s, 3H, 1-Me), 1.39 (d, J=7 Hz, 6H, $\operatorname{Pr}$ ); ${ }^{13} \mathrm{C}-\mathrm{NMR}$ $\left(\mathrm{CDCl}_{3}\right) \delta_{\mathrm{C}} 167.7$ (C, C-9), $151.2(\mathrm{C}, \mathrm{C}-4), 143.7$ (C, C-7), 142.1 (C, Ph), $140.9(\mathrm{CH}$, C-2), 140.6 (C, C-8a), 136.3 (CH, C-6), 135.4 (C, C-3a), 134.4 (CH, C-8), 130.3 (CH, C-5), 128.5 (CH, Ph), 128.3 (CH, Ph), 125.9 (CH, Ph), 124.3 (C, C-1), 116.1 (C, C-3), $51.1\left(\mathrm{CH}_{3}, \mathrm{OMe}\right), 41.9\left(\mathrm{CH}_{2}, \mathrm{C}-10\right), 37.9\left(\mathrm{CH},{ }^{\mathrm{i}} \mathrm{Pr}\right), 37.7\left(\mathrm{CH}_{2}, \mathrm{C}-11\right), 24.6\left(\mathrm{CH}_{3},{ }^{\mathrm{i}} \mathrm{Pr}\right)$, $12.9\left(\mathrm{CH}_{3}, 1-\mathrm{Me}\right)$; UV-Vis $\left(\mathrm{CH}_{2} \mathrm{Cl}_{2}\right) \lambda_{\max }: 574 \mathrm{~nm}$; IR (KBr): 2958, 2923, 2857, 1698, 1523, 1436, 1403, 1192, 1030, 748, $700 \mathrm{~cm}^{-1}$; HRMS: $m / z \mathrm{C}_{24} \mathrm{H}_{27} \mathrm{O}_{2}{ }^{+}[\mathrm{M}+\mathrm{H}]^{+}$, calcd 347.2011, found 347.2014.

(41): ${ }^{1} \mathrm{H}-\mathrm{NMR}:\left(\mathrm{CDCl}_{3}\right) \delta_{\mathrm{H}} 8.30(\mathrm{~s}, 1 \mathrm{H}, \mathrm{H}-8), 7.99(\mathrm{~s}, 1 \mathrm{H}, \mathrm{H}-2), 7.68(\mathrm{~d}, \mathrm{~J}=11 \mathrm{~Hz}, 1 \mathrm{H}$, H-6), 7.54 (d, J=11 Hz, 1H, H-5), 7.25-7.28 (m, 4H, Ph), 7.19 (m, 1H, Ph), 4.00 (s, 3H, OMe), 3.79 (t, J=9 Hz, 2H, H-11), 3.17 (sep, J=7 Hz, 1H, ${ }^{\mathrm{i}} \mathrm{Pr}$ ), 3.06 (t, J= $8 \mathrm{~Hz}$, 2H, H-12), 2.58 (s, 3H, 1-Me), $1.40\left(\mathrm{~d}, \mathrm{~J}=7 \mathrm{~Hz}, 6 \mathrm{H},{ }^{\mathrm{i}} \mathrm{Pr}\right) ;{ }^{13} \mathrm{C}-\mathrm{NMR}\left(\mathrm{CDCl}_{3}\right) \delta_{\mathrm{C}} 180.5$ (C, C-9), 166.7 (C, C-10), 154.4 (C, C-4), 147.9 (C, C-7), 144.7 (C, C-8a), 144.1 (CH, C-2), 141.8 (C, Ph), 139.2 (C, C-3a), 137.6 (CH, C-6), 139.4 (CH, C-8), 134.0 (CH, C-5), $128.6(\mathrm{CH}, \mathrm{Ph}), 126.0(\mathrm{C}, \mathrm{C}-1), 125.9(\mathrm{CH}, \mathrm{Ph}), 119.8$ (C, C-3), $52.6\left(\mathrm{CH}_{3}\right.$, OMe $), 43.0\left(\mathrm{CH}_{2}, \mathrm{C}-11\right), 38.14\left(\mathrm{CH}_{2}, \mathrm{C}-12\right), 38.09\left(\mathrm{CH},{ }^{\mathrm{i}} \mathrm{Pr}\right), 24.5\left(\mathrm{CH}_{3},{ }^{\mathrm{i}} \mathrm{Pr}\right), 13.1$ $\left(\mathrm{CH}_{3}, 1-\mathrm{Me}\right)$; UV-Vis $\left(\mathrm{CH}_{2} \mathrm{Cl}_{2}\right) \lambda_{\max }: 523 \mathrm{~nm}$; IR (KBr): $v_{\max } 2959,2923,2862,1733$, 1629, 1540, 1399, 1365, 1266, 1171, 745, $699 \mathrm{~cm}^{-1}$; HRMS: $m / z \mathrm{C}_{25} \mathrm{H}_{26} \mathrm{O}_{3} \mathrm{Na}^{+}$ $[\mathrm{M}+\mathrm{Na}]^{+}$, calcd 397.1780 , found 397.1783 . 


\section{4-(2-Phenylethyl)-7-isopropyl-1-methylazulene-3-carbaldehyde (42)}

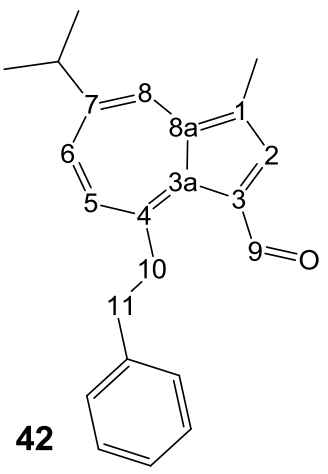

4-(2-Phenylethyl)-7-isopropyl-1-methylazulene (62 mg, 0.215 mmol) in DMF ( $5 \mathrm{~mL}$ ) was added to an RBF under standard conditions. Vilsmeier reagent was prepared by addition of DMF $(1 \mathrm{~mL})$ and $\mathrm{POCl}_{3}(0.1 \mathrm{~mL})$ under standard conditions and 0.25 $\mathrm{mL}$ (1.13 eq.) of this reagent was added to the reaction mixture, upon which a colour changed from blue to orange-red was observed. $\mathrm{NaOH}(1.2 \mathrm{~g}, 30 \mathrm{mmol})$ was dissolved in $\mathrm{H}_{2} \mathrm{O}(10$

$\mathrm{mL}$ ) and added to the reaction mixture. Organic material was extracted using $\mathrm{CH}_{2} \mathrm{Cl}_{2}$, followed by a further separation with hexanes/CuSO $4(\mathrm{aq})$ to remove residual DMF. Column chromatography (gradient of hexanes $\rightarrow \mathrm{CH}_{2} \mathrm{Cl}_{2}$ ) was performed to obtain 24 $\mathrm{mg}(36 \%)$ of the title compound as a purple solid. m.p. 85.9-88.6 ${ }^{\circ} \mathrm{C} ;{ }^{1} \mathrm{H}-\mathrm{NMR}:\left(\mathrm{CDCl}_{3}\right) \delta_{\mathrm{H}} 10.58$ (s, 1H, H-9), $8.31(\mathrm{~d}, \mathrm{~J}=2 \mathrm{~Hz}, 1 \mathrm{H}, \mathrm{H}-$ 8), 8.28 (2, 1H, H-2), 7.59 (dd, J=11 Hz, 2 Hz, 1H, H-6), 7.41 (d, J=11 Hz, 1H, H-5), 7.32 (t, J=8 Hz, 2H, Ph), 7.27-7.22 (complex m, J=8 Hz, 3H, Ph), 3.79 (t, J=8 Hz, 2H, H-10), 3.19 (t, J=8 Hz, 2H, H-11), 3.16 (sep, J=7 Hz, 1H, ${ }^{\mathrm{i}} \mathrm{Pr}$ ), 2.62 (s, 3H, 1-Me), $1.40\left(\mathrm{~d}, \mathrm{~J}=7 \mathrm{~Hz}, 6 \mathrm{H},{ }^{\mathrm{i}} \mathrm{Pr}\right) ;{ }^{13} \mathrm{C}-\mathrm{NMR}\left(\mathrm{CDCl}_{3}\right) \delta_{\mathrm{C}} 185.7(\mathrm{CH}, \mathrm{C}-9), 151.4(\mathrm{C}, \mathrm{C}-4)$, 147.0 (C, C-7), 143.8 (C, C-8a), 141.6 (CH, C-2), 140.9 (C, Ph), 138.2 (C, C-3a), 136.6 (CH, C-6), 135.3 (CH, C-8), 132.3 (CH, C-5), 128.6 (CH, Ph), 128.5 (CH, Ph), 127.1 (C, C-1), 126.3 (CH, Ph), 125.6 (C, C-3), $43.7\left(\mathrm{CH}_{2}, \mathrm{C}-10\right), 38.1\left(\mathrm{CH},{ }^{\mathrm{i}} \mathrm{Pr}\right), 37.7$ $\left(\mathrm{CH}_{2}, \mathrm{C}-11\right), 24.5\left(\mathrm{CH}_{3},{ }^{\mathrm{i}} \mathrm{Pr}\right), 13.2\left(\mathrm{CH}_{3}, 1-\mathrm{Me}\right)$; IR (KBr): 2959, 1659, 1626, 1541 , 1393, 1366, 1158, 720, $699 \mathrm{~cm}^{-1}$; HRMS: $m / z \mathrm{C}_{23} \mathrm{H}_{25} \mathrm{O}^{+}[\mathrm{M}+\mathrm{H}]^{+}$, calcd 317.1905, found 317.1906.

\section{4-(3-Bromopropyl)-7-isopropyl-1-methylazulene (38)}

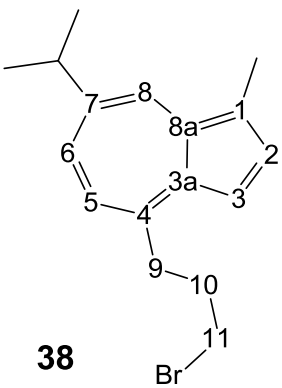

Guaiazulene $(98.7 \mathrm{mg}, 0.497 \mathrm{mmol})$ in THF $(8 \mathrm{~mL})$ was added to an RBF under standard conditions. LDA (2 mL THF, $0.2 \mathrm{~mL}$ diisopropylamine and $0.7 \mathrm{~mL} 2 \mathrm{M} n$-BuLi in cyclohexane) was made up under standard conditions and $1.4 \mathrm{~mL}$ (1.36 eq.) was added to the reaction mixture, which changed colour to orange. 1,2-Dibromoethane $(0.15 \mathrm{~mL}, 1.73 \mathrm{mmol})$ was added and the 
mixture returned to blue after approximately one minute. This reaction was left for a further 10 minutes before being quenched with $\mathrm{H}_{2} \mathrm{O}$. Organic material was extracted with $\mathrm{CH}_{2} \mathrm{Cl}_{2}$ and column chromatography on the residue was performed in hexanes to afford $32 \mathrm{mg}(21 \%)$ of the title product as a viscous blue oil.

${ }^{1} \mathrm{H}-\mathrm{NMR}:\left(\mathrm{CDCl}_{3}\right) \delta_{\mathrm{H}} 8.22$ (s, 1H, H-8), 7.67 (d, J=4 Hz, 1H, H-2), 7.45 (d, J=11 Hz, 1H, H-6), 7.34 (d, J=4 Hz, 1H, H-3), 7.05 (d, J=11 Hz, 1H, H-5), 3.49 (t, J=7 Hz, 2H, H-11), 3.33 (t, J=7 Hz, 2H, H-9), 3.10 (sep, J=7 Hz, 1H, ${ }^{\mathrm{i}} \mathrm{Pr}$ ), 2.69 (s, 3H, 1-Me), 2.41 (quin, J=7 Hz, 2H, H-10), $1.39\left(\mathrm{~d}, \mathrm{~J}=7 \mathrm{~Hz}, 6 \mathrm{H},{ }^{\mathrm{i}} \mathrm{Pr}\right) ;{ }^{13} \mathrm{C}-\mathrm{NMR}\left(\mathrm{CDCl}_{3}\right) \delta_{\mathrm{C}} 147.0(\mathrm{C}$, C-4), 140.2 (C, C-7), 137.0 (C, C-3a), 136.6 (CH, C-2), 136.3 (C, C-8a), 135.3 (CH, C-6), 133.5 (CH, C-8), 125.6 (C, C-1), 124.6 (CH, C-5), 112.4 (CH, C-3), 38.3 (CH, $\left.{ }^{\mathrm{i}} \mathrm{Pr}\right), 36.4\left(\mathrm{CH}_{2}, \mathrm{C}-9\right), 34.0\left(\mathrm{CH}_{2}, \mathrm{C}-10\right), 33.8\left(\mathrm{CH}_{2}, \mathrm{C}-11\right), 24.8\left(\mathrm{CH}_{3},{ }^{\mathrm{i}} \mathrm{Pr}\right), 13.0\left(\mathrm{CH}_{3}\right.$, $1-\mathrm{Me})$.

\section{4-(3-Propylthiol)-7-isopropyl-1-methylazulene (39)}

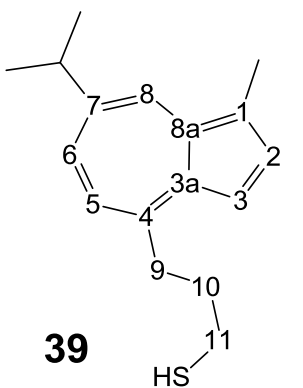

$38(32.4 \mathrm{mg}, 0.106 \mathrm{mmol})$ in $\mathrm{EtOH}(6 \mathrm{~mL})$ was added to an RBF under standard conditions. $\mathrm{Et}_{2} \mathrm{O}(0.5 \mathrm{~mL})$ was added to aid dissolution. Thiourea $(63.2 \mathrm{mg}, 0.83 \mathrm{mmol})$ was added to the reaction mixture, which was left to reflux overnight. $\mathrm{NaOH}$ (1.074 $\mathrm{g}$ in $10 \mathrm{~mL} \mathrm{H}_{2} \mathrm{O}$ ) was added to this reaction mixture, which was left to cool to r.t. for a further 6 hours. The organic layer was extracted using $\mathrm{CH}_{2} \mathrm{Cl}_{2}$, and purified by column chromatography (gradient of hexanes $\rightarrow \mathrm{CH}_{2} \mathrm{Cl}_{2}$ ) to afford $19 \mathrm{mg}(68 \%)$ of the title compound as a blue oil. ${ }^{1} \mathrm{H}-\mathrm{NMR}:\left(\mathrm{CDCl}_{3}\right) \delta_{\mathrm{H}} 8.21$ (s, 1H, H-8), 7.66 (d, J=4 Hz, 1H, H-2), 7.44 (d, J=11 Hz, 1H, H-6), 7.33 (d, J=4 Hz, 1H, H-3), 7.02 (d, J=11 Hz, 1H, H-5), 3.26 (t, J=8 Hz, 2H, H-9), 3.10 (sep, J=7 Hz, 1H, ${ }^{\mathrm{i}} \mathrm{Pr}$ ), 2.78 (t, J=7 Hz, 2H, H-11), 2.69 (s, 3H, 1-Me), 2.26 (quin, J=7 Hz, 2H, H-10), $1.38\left(\mathrm{~d}, \mathrm{~J}=7 \mathrm{~Hz}, 6 \mathrm{H},{ }^{\mathrm{i}} \mathrm{Pr}\right),{ }^{13} \mathrm{C}-\mathrm{NMR}\left(\mathrm{CDCl}_{3}\right) \delta_{\mathrm{C}} 147.9(\mathrm{C}$, C-4), 139.9 (C, C-7), 137.1 (C, C-3a), 136.5 (CH, C-2), 136.1 (C, C-8a), 135.2 (CH, C-6), 133.4 (CH, C-8), 125.4 (C, C-1), 124.6 (CH, C-5), 112.4 (CH, C-3), $38.8\left(\mathrm{CH}_{2}\right.$, C-11), $38.3\left(\mathrm{CH},{ }^{\mathrm{i}} \mathrm{Pr}\right), 36.6\left(\mathrm{CH}_{2}, \mathrm{C}-9\right), 30.4\left(\mathrm{CH}_{2}, \mathrm{C}-10\right), 24.8\left(\mathrm{CH}_{3},{ }^{\mathrm{i}} \mathrm{Pr}\right), 13.0\left(\mathrm{CH}_{3}\right.$, 1-Me); IR (KBr): $v_{\max } 2958,2924,2863,1642,1558,1463,1393,906,730 \mathrm{~cm}^{-1}$. 


\section{2-(7-Isopropyl-1-methyl azulen-4-yl)ethyl ethanoate (55)}

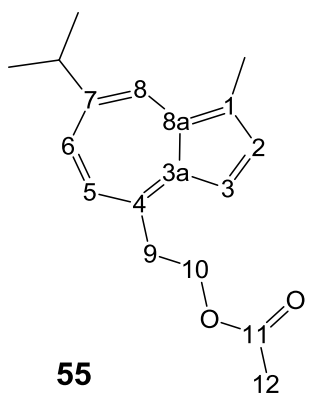

Guaiazulene (199.9 mg, $1.008 \mathrm{mmol}$ ) in $9 \mathrm{~mL}$ THF was added to an RBF under standard conditions. LDA (2 mL THF, $0.4 \mathrm{~mL}$ diisopropylamine and $1.4 \mathrm{~mL} 2 \mathrm{M} n$-BuLi in cyclohexane) was made under standard conditions, and $1.4 \mathrm{~mL}$ added to reaction mixture, whose colour changed from blue to orange. Cannulated formaldehyde into reaction mixture (as described

in the synthesis of 4-(2-hydroxyethyl)-7-isopropyl-1-methyl azulene) and a fast colour change back to blue was observed. $\mathrm{Ac}_{2} \mathrm{O}(1.0 \mathrm{~mL}, 10.6 \mathrm{mmol})$ was then added dropwise. The reaction was quenched with $\mathrm{H}_{2} \mathrm{O}$ and the organic material extracted with $\mathrm{CH}_{2} \mathrm{Cl}_{2}$. Rotary evaporation followed by evaporation under high-vaccuum was performed to remove $\mathrm{Ac}_{2} \mathrm{O} / \mathrm{AcOH}$, followed by column chromatography in $\mathrm{CH}_{2} \mathrm{Cl}_{2}$ to afford $76 \mathrm{mg}(29 \%)$ of the title compound as a blue oil.

${ }^{1} \mathrm{H}-\mathrm{NMR}:\left(\mathrm{CDCl}_{3}\right) \delta_{\mathrm{H}} 8.21(\mathrm{~d}, \mathrm{~J}=2 \mathrm{~Hz}, 1 \mathrm{H}, \mathrm{H}-8), 7.68(\mathrm{~d}, \mathrm{~J}=4 \mathrm{~Hz}, 1 \mathrm{H}, \mathrm{H}-2), 7.44(\mathrm{dd}$, J=11 Hz, 2 Hz, 1H, H-6), 7.34 (d, J=4 Hz, 1H, H-3), 7.03 (d, J=11 Hz, 1H, H-5), 4.49 (t, J=7 Hz, 2H, H-10), 3.48 (t, J=7 Hz, 2H, H-9), 3.09 (sep, J=7 Hz, 1H, ${ }^{\mathrm{i}} \operatorname{Pr}$ ), 2.68 (s, 3H, 1-Me), 2.05 (s, 3H, H-12), 1.38 (d, J=7 Hz, 6H, $\left.{ }^{\mathrm{i}} \mathrm{Pr}\right) ;{ }^{13} \mathrm{C}-\mathrm{NMR}\left(\mathrm{CDCl}_{3}\right) \delta_{\mathrm{C}} 171.1$ (C, C-11), 143.4 (C, C-4), 140.4 (C, C-7), 137.5 (C, C-3a), 137.0 (CH, C-2), 136.3 (C, C-8a), 135.1 (CH, C-6), 133.6 (CH, C-8), 125.6 (C, C-1), 124.7 (CH, C-5), 112.5 (CH, C-3), $64.7\left(\mathrm{CH}_{2}, \mathrm{C}-10\right), 38.3\left(\mathrm{CH},{ }^{\mathrm{i}} \mathrm{Pr}\right), 37.3\left(\mathrm{CH}_{2}, \mathrm{C}-9\right), 24.8\left(\mathrm{CH}_{3},{ }^{\mathrm{i}} \mathrm{Pr}\right), 21.1$ $\left(\mathrm{CH}_{3}, \mathrm{C}-12\right), 13.0\left(\mathrm{CH}_{3}, 1-\mathrm{Me}\right)$; IR (KBr): $v_{\max } 2958,2873,1742,1556,1462,1385$, 1238, 1024; HRMS: $m / z: \mathrm{C}_{18} \mathrm{H}_{22} \mathrm{O}_{2} \mathrm{Na}^{+}[\mathrm{M}+\mathrm{Na}]^{+}$, calcd 293.1517, found 293.1517.

\section{N-(2-(7-Isopropyl-1-methylazulen-4-yl)ethyl)piperidine (57)}

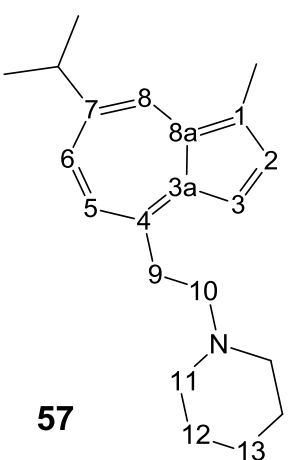

To a flame-dried RBF with stirrer, piperidine $(0.6 \mathrm{~mL}, 6.07$ mmol) in DMF $(2.4 \mathrm{~mL})$ was added, followed by 2-(7isopropyl-1-methyl azulen-4-yl)ethyl acetate $(16.1 \mathrm{mg}, 0.062$ mmol). The reaction was left for 60 hours, over which period no colour change was observed. A separation was performed using hexanes $/ \mathrm{CuSO}_{4}(\mathrm{aq})$ to remove $\mathrm{DMF}$ from the reaction mixture. Column chromatography was performed on this residue

(gradient in hexanes $\rightarrow \mathrm{CH}_{2} \mathrm{Cl}_{2} \rightarrow \mathrm{Et}_{2} \mathrm{O}$ ) to afford $5.9 \mathrm{mg}$ (34\%) of the title compound as a blue oil. 
${ }^{1} \mathrm{H}-\mathrm{NMR}:\left(\mathrm{CDCl}_{3}\right) \delta_{\mathrm{H}} 8.18(\mathrm{~s}, 1 \mathrm{H}, \mathrm{H}-8), 7.65$ (d, J=4 Hz, 1H, H-2), 7.42 (d, J=11 Hz, 1H, H-6), 7.34 (d, J=4 Hz, 1H, H-3), 7.03 (d, J=11 Hz, 1H, H-5), 3.41 (m, J=8 Hz, 2H, H-9), 3.07 (sep, J=7 Hz, 1H, ${ }^{\mathrm{i}} \mathrm{Pr}$ ), 2.85 (m, J=8 Hz, 2H, H-10), 2.66 (s, 3H, 1-Me), 2.61 (broad s, 4H, H-11), 1.70 (quin, J=6 Hz, 4H, H-12), 1.50 (m, 2H, H-13), 1.36 (d, $\left.\mathrm{J}=7 \mathrm{~Hz}, 6 \mathrm{H},{ }^{\mathrm{i}} \mathrm{Pr}\right) ;{ }^{13} \mathrm{C}-\mathrm{NMR}\left(\mathrm{CDCl}_{3}\right) \delta_{\mathrm{C}} 146.7(\mathrm{C}, \mathrm{C}-4), 140.0(\mathrm{C}, \mathrm{C}-7), 137.3(\mathrm{C}, \mathrm{C}-$ 3a), 136.5 (CH, C-2), 135.9 (C, C-8a), 135.3 (CH, C-6), 133.4 (CH, C-8), 125.3 (C, C-1), 125.0 (CH, C-5), 112.2 (CH, C-3), $60.4\left(\mathrm{CH}_{2}, \mathrm{C}-10\right), 54.3\left(\mathrm{CH}_{2}, \mathrm{C}-11\right), 38.2$ (CH, $\left.{ }^{\mathrm{i}} \mathrm{Pr}\right), 35.4\left(\mathrm{CH}_{2}, \mathrm{C}-9\right), 25.8\left(\mathrm{CH}_{2}, \mathrm{C}-12\right), 24.7\left(\mathrm{CH}_{3},{ }^{\mathrm{i}} \mathrm{Pr}\right), 24.2\left(\mathrm{CH}_{2}, \mathrm{C}-13\right), 12.9$ $\left(\mathrm{CH}_{3}, 1-\mathrm{Me}\right)$; IR (KBr): $v_{\max } 2934,2861,2800,1555,1442,1387,1113,1027,776$; HRMS: $m / z \mathrm{C}_{17} \mathrm{H}_{30} \mathrm{~N}[\mathrm{M}+\mathrm{H}]^{+}$, calcd 296.2378, found 296.2379.

\section{Guaiazulene-functionalised Merrifield resin (50)}

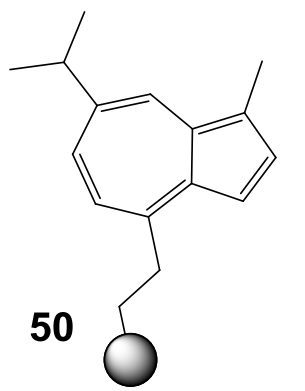

Guaiazulene $(212.0 \mathrm{mg}, 1.069 \mathrm{mmol})$ in THF $(10 \mathrm{~mL})$ was added to an RBF under standard conditions. LDA $(0.35 \mathrm{~mL}$ diisopropylamine, $1 \mathrm{~mL}$ THF and $1.3 \mathrm{~mL}$ of $2 \mathrm{M} n$-BuLi in cyclohexane) was made up under standard conditions. $1.7 \mathrm{~mL}$ (1.7 eq.) of this solution was added to the reaction mixture and a colour change from blue to orange was observed. A second

flame-dried RBF containing Merrifield resin (2\% crosslinked, 33\% functionalised, 100-200 micron) (358.1 mg, $0.992 \mathrm{mmol})$ in toluene $5 \mathrm{~mL}$ was sonicated for one minute in an attempt to maximise swelling. The orange reaction mixture was cannulated across into the Merrifield suspension under argon, and an immediate colour change to blue was observed in the Merrifield suspension. Once addition was complete, mixture was blue-green. A small amount of paraformaldehyde was added in order to trap remaining guaiazulenide anion. A separation was performed with $\mathrm{H}_{2} \mathrm{O} /$ toluene and the organic fraction \& beads were filtered and washed with the solvents dichloromethane, toluene, acetone, methanol and water. $438.2 \mathrm{mg}$ of the blue-green resin $\mathbf{5 0}$ was obtained. The filtrate was reduced under rotary evaporation and purified in column chromatography to obtain guaiazulene (28 mg, 13\%) and the alcohol 36 (41 mg, 17\%), plus several minor blue products totalling $17 \mathrm{mg}$. 


\section{Guaiazulene methyl ketoester functionalised Merrifield resin (51)}

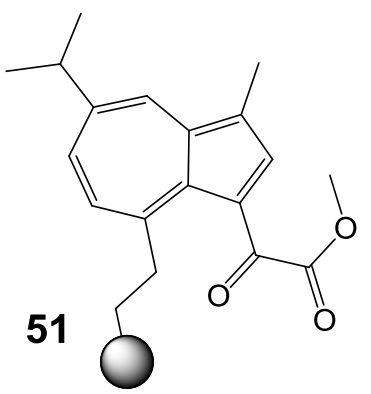

50 (52.6 $\mathrm{mg}, 0.12 \mathrm{mmol}$ ) was added to a flame-dried RBF, followed by $\mathrm{CH}_{2} \mathrm{Cl}_{2}(12 \mathrm{~mL})$. $(\mathrm{COCl})_{2}(0.1 \mathrm{~mL}, 1.18 \mathrm{mmol})$ was added dropwise, whereupon a subtle colour change from dark blue to dark brown was observed. This was followed by addition of $\mathrm{MeOH}(0.5 \mathrm{~mL}, 12.3 \mathrm{mmol})$ after 30 seconds and pyridine $(0.6 \mathrm{~mL})$ after two minutes. Resin was filtered and washed with dichloromethane, acetone, methanol and water, and dried under a highvaccuum. $\mathbf{5 7} \mathrm{mg}$ of $\mathbf{5 1}$ was obtained as a dark brown resin.

\section{Guaiazulene aldehyde functionalised Merrifield resin (52)}

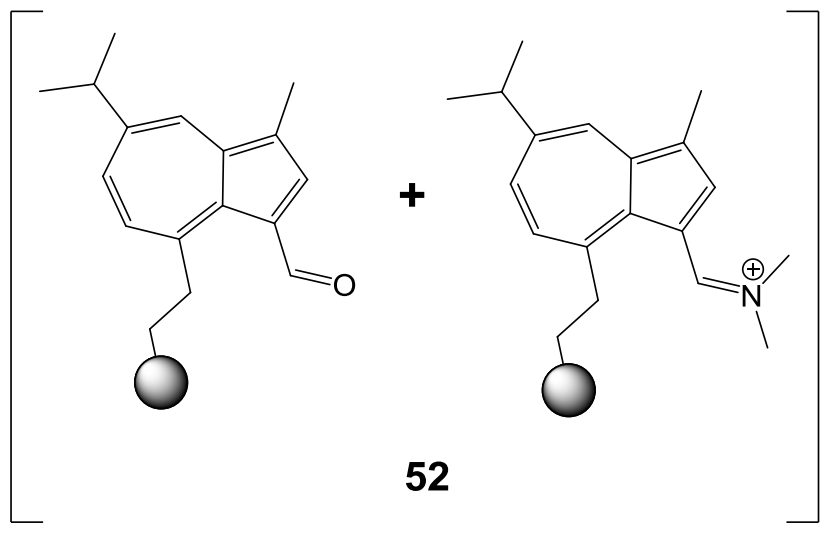

50 (48.4 mg, $0.11 \mathrm{mmol}$ ) was added to a flame-dried $\mathrm{RBF}$ followed by addition of toluene (5 mL) and one minute of sonication. Vilsmeier reagent $\left(0.15 \mathrm{~mL} \mathrm{POCl}_{3}\right.$ and $\left.1 \mathrm{~mL} \mathrm{DMF}\right)$ was made up under standard conditions, and $0.1 \mathrm{~mL}(0.21$

mmol) was added to the reaction suspension. A colour change in the resin was observed to dark orange. $\mathrm{NaOH}(3.46 \mathrm{~g})$ in $\mathrm{H}_{2} \mathrm{O}(10 \mathrm{~mL})$ was added to the reaction vessel, which was sonicated for 10 minutes. The resin was filtered and washed with dichloromethane, acetone, methanol and water, and dried under high-vaccuum. $50 \mathrm{mg}$ of $\mathbf{5 2}$ was obtained as a dark brown resin.

\section{4-(Trimethylsilylmethylene)-7-isopropyl-1-methylazulene (69)}

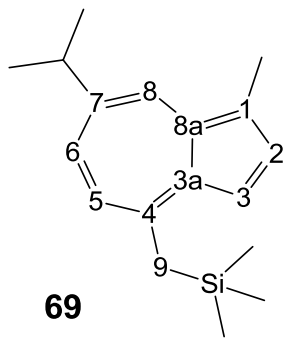

Guaiazulene (223.2 mg, $1.126 \mathrm{mmol}$ ) in $10 \mathrm{~mL}$ THF was added to a heatgun-dried RBF with stirrer. LDA (4 mL THF, $0.8 \mathrm{~mL}$ Diisopropylamine and $2.8 \mathrm{~mL}$ of $2 \mathrm{M} n$-BuLi in cyclohexane) was made under standard conditions, and $1.9 \mathrm{~mL}$ (1.2 eq.) added to reaction mixture, which changed colour from blue to orange.

TMS-Cl (0.2 mL, $1.575 \mathrm{mmol})$ was added, and an instantaneous colour change back to blue was observed. Reaction was quenched with $\mathrm{H}_{2} \mathrm{O}$ and organic material extracted with $\mathrm{CH}_{2} \mathrm{Cl}_{2}$. Column chromatography was performed on this organic 
residue using hexanes to afford $203 \mathrm{mg}(67 \%)$ of the title compound as a viscous blue oil.

${ }^{1} \mathrm{H}-\mathrm{NMR}:\left(\mathrm{CDCl}_{3}\right) \delta_{\mathrm{H}} 8.17$ (s, 1H, H-8), 7.56 (d, J=4 Hz, 1H, H-2), 7.37 (dd, J=11 Hz, 2 Hz, 1H, H-6), 7.15 (d, J=4 Hz, 1H, H-3), 6.86 (d, J=11 Hz, 1H, H-5), 3.08 (sep, J=7 $\mathrm{Hz}, 1 \mathrm{H},{ }^{\mathrm{i}} \mathrm{Pr}$ ), 2.74 (s, 2H, H-9), 2.67 (s, 3H, 1-Me), 1.38 (d, J=7 Hz, 6H, ${ }^{\mathrm{i}} \mathrm{Pr}$ ), 0.05 (s, 9H, TMS); ${ }^{13} \mathrm{C}-\mathrm{NMR}\left(\mathrm{CDCl}_{3}\right) \delta_{\mathrm{C}} 149.7$ (C, C-4), 139.2 (C, C-7), 136.8 (C, C-3a), 135.8 (CH, C-2), 135.6 (C, C-8a), 135.4 (CH, C-6), 133.6 (CH, C-8), 125.8 (C, C-1), $125.7(\mathrm{CH}, \mathrm{C}-5), 114.2(\mathrm{CH}, \mathrm{C}-3), 38.8\left(\mathrm{CH},{ }^{\mathrm{i}} \mathrm{Pr}\right), 30.9\left(\mathrm{CH}_{2}, \mathrm{C}-9\right), 25.6\left(\mathrm{CH}_{3},{ }^{\mathrm{i}} \mathrm{Pr}\right)$, $13.8\left(\mathrm{CH}_{3}, 1-\mathrm{Me}\right), 0.00\left(\mathrm{CH}_{3}, \mathrm{TMS}\right)$; UV-Vis $\left(\mathrm{CH}_{2} \mathrm{Cl}_{2}\right) \lambda_{\max }: 655 \mathrm{~nm}$; IR (KBr): $v_{\max }$ 2957, 2866, 1687, 1551, 1423, 1387, 1248, 1140, $840 \mathrm{~cm}^{-1}$; HRMS: $m / z \mathrm{C}_{17} \mathrm{H}_{27} \mathrm{Si}$ $[\mathrm{M}+\mathrm{H}]^{+}$, calcd 271.1882, found 271.1880.

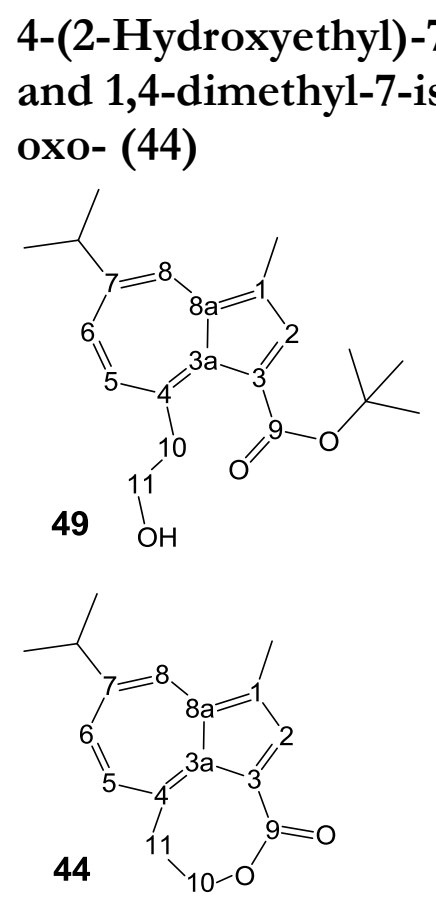

Ester 47 (54.3 mg, $0.182 \mathrm{mmol})$ in THF (5 mL) was added to an RBF under standard conditions. LDA was made up under standard conditions $(2 \mathrm{~mL}$ THF, $0.2 \mathrm{~mL}$ diisopropylamine and $0.7 \mathrm{~mL}$ of $2 \mathrm{M} \quad n$-BuLi in cyclohexane) and $0.35 \mathrm{~mL}$ was added to the reaction mixture, which changed colour from indigo to yellow. Paraformaldehyde (86 mg, $2.9 \mathrm{mmol}$ ) was cannulated into the reaction mixture as formaldehyde gas, and a colour change back to indigo was observed. $\mathrm{H}_{2} \mathrm{O}$ was added and the organic layer extracted using $\mathrm{CH}_{2} \mathrm{Cl}_{2}$. Organic fraction was reduced by rotary evaporation and purified using

column chromatography (gradient of hexanes $\rightarrow \mathrm{CH}_{2} \mathrm{Cl}_{2} \rightarrow \mathrm{Et}_{2} \mathrm{O}$ ) to afford $15 \mathrm{mg}$ (25\% yield) of $\mathbf{4 9}$ as an indigo oil and $4 \mathrm{mg}$ (8\% yield) of $\mathbf{4 4}$ as a magenta substance.

(49): ${ }^{1} \mathrm{H}-\mathrm{NMR}:\left(\mathrm{CDCl}_{3}\right) \delta_{\mathrm{H}} 8.22(\mathrm{~d}, \mathrm{~J}=2 \mathrm{~Hz}, 1 \mathrm{H}, \mathrm{H}-8), 7.95$ (s, 1H, H-2), 7.57 (dd, $\mathrm{J}=11 \mathrm{~Hz}, 2 \mathrm{~Hz}, 1 \mathrm{H}, \mathrm{H}-6), 7.32$ (d, J=11 Hz, 1H, H-5), 4.17 (t, J=6 Hz, 2H, H-11), 3.51 (t, J=6 Hz, 2H, H-10), 3.10 (sep, J=7 Hz, 1H, ${ }^{\mathrm{i}} \mathrm{Pr}$ ), 2.58 (s, 3H, 1-Me), 1.64 (s, 9H, $\left.{ }^{\mathrm{t}} \mathrm{Bu}\right), 1.36\left(\mathrm{~d}, \mathrm{~J}=7 \mathrm{~Hz}, 6 \mathrm{H},{ }^{\mathrm{i}} \mathrm{Pr}\right) ;{ }^{13} \mathrm{C}-\mathrm{NMR}\left(\mathrm{CDCl}_{3}\right) \delta_{\mathrm{C}} 168.1$ (C, C-9), 148.7 (C, C-4), 143.7 (C, C-7), 141.6 (CH, C-2), 140.4 (C, C-8a), 137.6 (C, C-3a), 136.4 (CH, C-6), 134.5 (CH, C-8), 129.6 (CH, C-5), 124.4 (C, C-1), 117.4 (C, C-3), 80.8 (C, $\left.{ }^{\mathrm{t}} \mathrm{Bu}\right), 63.7$ 
$\left(\mathrm{CH}_{2}, \mathrm{C}-11\right), 41.8\left(\mathrm{CH}_{2}, \mathrm{C}-10\right), 37.9\left(\mathrm{CH},{ }^{\mathrm{i}} \mathrm{Pr}\right), 28.4\left(\mathrm{CH}_{3},{ }^{\mathrm{t}} \mathrm{Bu}\right), 24.6\left(\mathrm{CH}_{3},{ }^{\mathrm{i}} \mathrm{Pr}\right), 13.0$ $\left(\mathrm{CH}_{3}, 1-\mathrm{Me}\right)$

(44): ${ }^{1} \mathrm{H}-\mathrm{NMR}:\left(\mathrm{CDCl}_{3}\right) \delta_{\mathrm{H}} 8.32(\mathrm{~d}, \mathrm{~J}=2 \mathrm{~Hz}, 1 \mathrm{H}, \mathrm{H}-8), 8.32(\mathrm{~s}, 1 \mathrm{H}, \mathrm{H}-2), 7.60(\mathrm{dd}$, $\mathrm{J}=11 \mathrm{~Hz}, 2 \mathrm{~Hz}, 1 \mathrm{H}, \mathrm{H}-6), 7.23$ (d, J=11 Hz, 1H, H-5), 4.64 (t, J=5 Hz, 2H, H-10), 3.62 (broad s, 2 Hz, H-11), 3.17 (sep, J=7 Hz, 1H, ${ }^{\mathrm{i}} \mathrm{Pr}$ ), 2.63 (s, 3H, 1-Me), 1.40 (d, J=7 $\left.\mathrm{Hz}, 6 \mathrm{H},{ }^{\mathrm{i}} \mathrm{Pr}\right) ;{ }^{13} \mathrm{C}-\mathrm{NMR}\left(\mathrm{CDCl}_{3}\right) \delta_{\mathrm{C}} 168.4$ (C, C-9), 147.8 (C, C-4), 145.9 (C, C-7), 144.1 (CH, C-2), 141.7 (C, C-8a), 136.2 (CH, C-6), 135.6 (CH, C-8), 135.5 (C, C-3a), 129.0 (CH, C-5), 127.0 (C, C-1), 114.4 (C, C-3), $66.0\left(\mathrm{CH}_{2}, \mathrm{C}-10\right), 41.4\left(\mathrm{CH}_{2}, \mathrm{C}-11\right)$, $38.3\left(\mathrm{CH},{ }^{\mathrm{i}} \mathrm{Pr}\right), 24.6\left(\mathrm{CH}_{3},{ }^{\mathrm{i}} \mathrm{Pr}\right), 12.7\left(\mathrm{CH}_{3}, 1-\mathrm{Me}\right)$; UV-Vis $\left(\mathrm{CH}_{2} \mathrm{Cl}_{2}\right) \lambda_{\max }: 555 \mathrm{~nm}$; IR $(\mathrm{KBr}): v_{\max } 2950,2925,1673,1419,1224,1001,770 \mathrm{~cm}^{-1}$; HRMS: $m / z \mathrm{C}_{17} \mathrm{H}_{19} \mathrm{O}_{2}{ }^{+}$ $\left[\mathrm{M}+\mathrm{H}^{+}\right]$, calcd 255.1385, found 255.1386.

\section{Bis-9,9'(7-isopropyl-1-methyl azulen-4-yl) isopropyl alcohol (64) and}

Bis-9,9'(7-isopropyl-1-methyl azulen-4-yl) isopropyl formate (65)

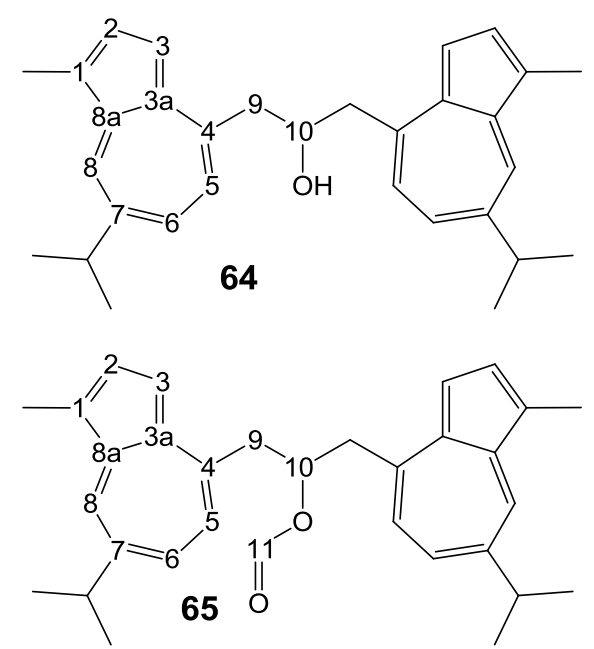

Guaiazulene (215 mg, $1.084 \mathrm{mmol}$ ) in $9 \mathrm{~mL}$ THF was added to an RBF under standard conditions. LDA (2mL THF, $0.8 \mathrm{~mL}$ diisopropylamine and $2.9 \mathrm{~mL} 2 \mathrm{M} n$-BuLi in cyclohexane) was made under standard conditions, and $1.4 \mathrm{~mL}$ (1.31 eq.) added to the reaction mixture, which changed colour to orange. Ethyl formate $(0.4 \mathrm{~mL}, 4.95$ mmol) was added to this mixture, and reaction mixture changed colour to blue-green. $\mathrm{H}_{2} \mathrm{O}$ was added to quench the reaction, and separation was

performed with $\mathrm{H}_{2} \mathrm{O} / \mathrm{CH}_{2} \mathrm{Cl}_{2}$ followed by column chromatography (gradient of hexanes $\rightarrow \mathrm{CH}_{2} \mathrm{Cl}_{2}$ ) to afford $69 \mathrm{mg}$ (30\%) of bis-9,9'(7-isopropyl-1-methyl azulen-4yl) isopropyl alcohol as a viscous blue oil and $80 \mathrm{mg}$ (32\%) of bis-9,9'(7-isopropyl-1methyl azulen-4-yl) isopropyl formate as a viscous blue oil.

(64): ${ }^{1} \mathrm{H}-\mathrm{NMR}:\left(\mathrm{CDCl}_{3}\right) \delta_{\mathrm{H}} 8.22(\mathrm{~d}, \mathrm{~J}=2 \mathrm{~Hz}, 2 \mathrm{H}, \mathrm{H}-8), 7.65$ (d, J=4 Hz, 2H, H-2), 7.44 (dd, J=11 Hz, 2 Hz, H-6), 7.23 (d, J=4 Hz, 2H, H-3), 7.06 (d, J=10 Hz, 2H, H-5), 4.65 (m, 1H, H-10), 3.48 (dd, J=13 Hz, 5 Hz, 2H, H-9a), 3.42 (dd, J=13 Hz, 8 Hz, 2H, $\mathrm{H}-9 \mathrm{~b}) 3.10$ (sep, J=7 Hz, 2H, ${ }^{\mathrm{i}} \mathrm{Pr}$ ), 2.68 (s, 6H, 1-Me), 1.38 (d, J=7 Hz, 12H, ${ }^{\mathrm{i}} \mathrm{Pr}$ ); ${ }^{13} \mathrm{C}-$ NMR $\left(\mathrm{CDCl}_{3}\right) \delta_{\mathrm{C}} 144.6$ (C, C-4), 140.3 (C, C-7), 138.0 (C, C-3a), 136.8 (CH, C-2), 136.3 (C, C-8a), 135.1 (CH, C-6), 133.6 (CH, C-8), 125.6 (C, C-1), 125.1 (CH, C-5), 112.6 (CH, C-3), 74.1 (CH, C-10), $46.0\left(\mathrm{CH}_{2}, \mathrm{C}-9\right), 38.3\left(\mathrm{CH},{ }^{\mathrm{i}} \mathrm{Pr}\right), 24.8\left(\mathrm{CH}_{3},{ }^{\mathrm{i}} \mathrm{Pr}\right)$, 
$13.0\left(\mathrm{CH}_{3}, 1-\mathrm{Me}\right)$; UV-Vis $\left(\mathrm{CH}_{2} \mathrm{Cl}_{2}\right) \lambda_{\max }: 608 \mathrm{~nm}$; IR (KBr): $v_{\max } 3291,2958,2862$, 1553, 1525, 1462, 1388, 1023, 919, $782 \mathrm{~cm}^{-1}$; HRMS: $m / z \mathrm{C}_{31} \mathrm{H}_{36} \mathrm{ONa}^{+}[\mathrm{M}+\mathrm{Na}]^{+}$, Calcd 447.2664, found 447.2667.

(65): ${ }^{1} \mathrm{H}-\mathrm{NMR}:\left(\mathrm{CDCl}_{3}\right) \delta_{\mathrm{H}} 8.19(\mathrm{~s}, 2 \mathrm{H}, \mathrm{H}-8), 7.85$ (s, 1H, H-11), 7.60 (d, J=4 Hz, H2), 7.42 (d, J=11 Hz, 2H, H-6), 7.12 (d, J=4 Hz, 2H, H-3), 7.02 (d, J=11 Hz, 2H, H-5), 5.92 (quin, J=7 Hz, 1H, H-10), 3.60 (dd, J=14 Hz, 8 Hz, 2H, H-9a), 3.42 (dd, J=13 Hz, $6 \mathrm{~Hz}, 2 \mathrm{H}, \mathrm{H}-9 \mathrm{~b}$ ), 3.09 (sep, J=7 Hz, 2H, $\mathrm{Pr}$ ), 2.67 (s, 6H, 1-Me), 1.38 (d, J=7 Hz, 12H); ${ }^{13} \mathrm{C}-\mathrm{NMR}\left(\mathrm{CDCl}_{3}\right) \delta_{\mathrm{C}} 160.8(\mathrm{CH}, \mathrm{C}-11), 142.8$ (C, C-4), 140.4 (C, C-7), 137.7 (C, C-3a), 137.1 (CH, C-2), 136.5 (C, C-8a), 134.8 (CH, C-6), 133.5 (CH, C-8), 125.6 (C, C-1), 124.9 (CH, C-5), 112.7 (CH, C-3), 76.0 (CH, C-10), $42.6\left(\mathrm{CH}_{2}, \mathrm{C}-9\right), 38.3$ (CH, $\left.{ }^{\mathrm{i}} \mathrm{Pr}\right), 24.7\left(\mathrm{CH}_{3},{ }^{\mathrm{i}} \mathrm{Pr}\right), 13.0\left(\mathrm{CH}_{3}, 1-\mathrm{Me}\right)$; UV-Vis $\left(\mathrm{CH}_{2} \mathrm{Cl}_{2}\right) \lambda_{\max }: 611 \mathrm{~nm}$; IR (KBr): $v_{\max } 2957,1718,1638,1459,1387,1173$, 736; HRMS: $m / z \mathrm{C}_{32} \mathrm{H}_{36} \mathrm{O}_{2} \mathrm{Na}^{+}$ $[\mathrm{M}+\mathrm{Na}]^{+}$, calcd 475.2613, found 475.2605

(4-(2-Hydroxyethyl)-7-isopropyl-1-methylazulen-3-yl) dimethylsulfonium chloride (67)

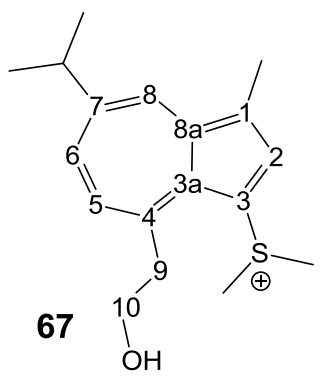
DMSO (50 $\mu \mathrm{L}, 0.703 \mathrm{mmol})$ in $2 \mathrm{~mL} \mathrm{CH}_{2} \mathrm{Cl}_{2}$ was added to an RBF under standard conditions. Reaction vessel cooled down to $-78^{\circ} \mathrm{C}$, and $(\mathrm{COCl})_{2}(40 \mu \mathrm{L}, 0.466 \mathrm{mmol})$ was added dropwise. After 20 minutes, 4-(2-hydroxyethyl)-7-isopropyl-1-methyl azulene (88.7 $\mathrm{mg}, 0.388 \mathrm{mmol}$ ) dissolved in $4 \mathrm{~mL} \mathrm{CH}_{2} \mathrm{Cl}_{2}$ was added. An instantaneous colour change to purple was observed.

$\mathrm{NEt}_{3}(160 \mu \mathrm{L}, 1.148 \mathrm{mmol})$ was added, and several separations were attempted using a $\mathrm{H}_{2} \mathrm{O} / \mathrm{CH}_{2} \mathrm{Cl}_{2}$ solvent system. The purple product is highly polar, and several extractions using $\mathrm{CH}_{2} \mathrm{Cl}_{2}$ were not entirely successful at removing the purple colour from the aqueous layer. Aqueous layer was reduced via rotary evaporation and the purple substance was extracted with $\mathrm{CH}_{2} \mathrm{Cl}_{2}$. A column was performed (gradient of $\mathrm{CH}_{2} \mathrm{Cl}_{2} \rightarrow \mathrm{Et}_{2} \mathrm{O}$ ) to remove residual blue starting material from the purple product. The remaining purple material was removed from silica using $\mathrm{MeOH}$ and reduced via rotary evaporation to afford the title compound (149 $\mathrm{mg}$ ) as an inseparable nonstoichiometric mixture with $\mathrm{HNEt}_{3}{ }^{+}(\sim 3.3$ equivalents) and DMSO ( 4.3 equivalents). ${ }^{1} \mathrm{H}-\mathrm{NMR}:\left(\mathrm{CDCl}_{3}\right) \delta_{\mathrm{H}} 8.25(\mathrm{~d}, \mathrm{~J}=2 \mathrm{~Hz}, 1 \mathrm{H}, \mathrm{H}-8), 8.23$ (s, 1H, H-2), 7.66 (dd, J=11 Hz, 2 Hz, 1H, H-6), 7.40 (d, J=11 Hz, 1H, H-5), 4.01 (t, J=7 Hz, 2H, H-10), 3.64 (t, J=7 Hz, 2H, H-9), 3.38 (s, 6H, S-Me), 3.07 (sep, J=7 Hz, 1H, ${ }^{\mathrm{i}} \mathrm{Pr}$ ), 3.03 (dq, J=7 Hz, 5 Hz, 
20H, $\mathrm{NEt}_{3}$ ), 2.55 (s, 3H, 1-Me), 2.52 (s, 26 H, DMSO), 1.30 (t, J=8 Hz, 30H), 1.27 (d, $\left.\mathrm{J}=7 \mathrm{~Hz}, 6 \mathrm{H},{ }^{\mathrm{i}} \mathrm{Pr}\right){ }^{13} \mathrm{C}-\mathrm{NMR}\left(\mathrm{CDCl}_{3}\right) \delta_{\mathrm{C}} 148.6(\mathrm{C}, \mathrm{C}-4), 147.2(\mathrm{C}, \mathrm{C}-7), 141.9(\mathrm{C}, \mathrm{C}-$ 8a), 139.5 (C, C-3a), 138.4 (CH, C-6), 136.7 (CH, C-8), 135.9 (CH, C-2), 133.5 (CH, C-5), 128.8 (C, C-1), 98.9 (C, C-3), $63.1\left(\mathrm{CH}_{2}, \mathrm{C}-10\right), 45.9\left(\mathrm{CH}_{2}, \mathrm{NEt}_{3}\right), 42.9\left(\mathrm{CH}_{2}\right.$, C-9), 40.9 ( $\mathrm{CH}_{3}$, DMSO), 37.9 (CH, $\left.{ }^{\mathrm{i}} \mathrm{Pr}\right), 33.8\left(\mathrm{CH}_{3}, \mathrm{~S}-\mathrm{Me}\right), 24.4\left(\mathrm{CH}_{3},{ }^{\mathrm{i}} \mathrm{Pr}\right), 13.2$ $\left(\mathrm{CH}_{3}, 1-\mathrm{Me}\right), 8.6\left(\mathrm{CH}_{3}, \mathrm{NEt}_{3}\right)$ 


\section{References}

1. Yu, Y.-Y.; Fu, Y.; Liu, L.; Guo, Q.-X. Acta Chim. Sinica 2007, 1014-1022.

2. Liu, R. S. H. J. Chem. Ed. 2002, 183-185.

3. Gordon, M. Chem. Rev. 1951, 50, 127-200.

4. Gutman, I.; Trinajsti, N. Can. J. Chem. 1975, 1789-1794.

5. Shevyakov, S. V.; Li, H.; Muthyala, R.; Asato, A. E.; Croney, J. C.; Jameson, D. M.; Liu, R. S. H. J. Phys. Chem. 2003, 107.

6. Shevyakov, S. V.; Li, H.; Muthyala, R.; Asato, A. E.; Croney, J. C.; Jameson, D. M.; Liu, R. S. H. Journal of Physical Chemistry 2003, 3295-3299.

7. Zahradnik, R. Angew. Chem. 1965, 1039-1050.

8. $\quad$ Liu, R. S. H.; Muthyala, R. S.; Wang, X.-s.; Asato, A. E. Org. Lett. 1999, 269271.

9. Razus, A. C.; Birzan, L.; Nae, S.; Lehadus, O. L.; Pavel, C.; Costan, O. ARKIVOC 2004, 71-85.

10. Hafner, K.; Bernhard, C.; Muller, R. J. Liebigs. Ann. 1961, 35-41.

11. Shoji, T.; Ito, S.; Watanabe, M.; Toyota, K.; Yasunamid, M.; Moritaa, N. Tet. Lett. 2007, 3009-3012.

12. Franke, H.; Muhlstadt, M. J. Prakt. Chem. 1967, 249-261.

13. Garner, C. M. J. Chem. Ed. 2005, 82, 1686-1688.

14. Arthur G . Anderson, J.; Cowles, E. J.; Tazuma, J. J.; Nelson, J. A.

15. Hafner, K.; Weldes, H. J. Liebigs. Ann. 1957, 90-99.

16. Aumüller, I.; Lindhorst, T. K. Eur. J. Org. Chem. 2006, 1103-1108.

17. Lemal, D. M.; Goldman, G. D. J. Chem. Ed. 1988, 923-925.

18. Hafner, K.; Kaiser, H. Org. Synth. 1973, 1088.

19. Hafner, K.; Pelster, H.; Schneider, J. J. Liebigs. Ann. 1961, 63-80.

20. Ito, S.; Morita, N.; Asao, T. Bull. Chem. Soc. Jap. 1995, 1409-1436.

21. Ito, S.; Akimoto, K.; Kawakami, J.; Tajiri, A.; Shoji, T.; Satake, H.; Morita, N. J. Org. Chem. 2007, 162-172.

22. Ito, S.; Terazono, T.; Kubo, T.; Okujima, T.; Morita, N.; Murafuji, T.; Sugihara, Y.; Fujimori, K.; Kawakamia, J.; Tajiria, A. Tet. Lett. 2004, 25, 5357-5366.

23. Baran, P. S.; Maimone, T. J.; Richter, J. M. Nature 2007, 446, 404-408.

24. Schelhaas, M.; Waldmann, H. Angew. Chem. 1996, 35, 2056-2083.

25. Wall, P. E. Thin-Layer Chromatography A modern practical approach; The Royal Society of Chemistry, 2005.

26. Chen, S.-H.; Hsu, J.-L.; Lin, F.-S. Anal. Chem. 2008, 80.

27. Walker, J. M. Methods in Molecular Biology 1997, 64.

28. Tun-Kyi, A.; Schwyzer, R. Helv. Chim. Acta 1976, 59, 1642-1646.

29. Timmer, M. S. M.; Stocker, B. L.; Northcote, P. T.; Burkett, B. A. Tet. Lett. 2009, 50, 7199-7204.

30. Vaino, A. R.; Janda, K. D. J. Comb. Chem. 2000, 2, 579-596.

31. Burkett, B. A. COSMOS 2008, 4, 17-37.

32. Retrieved 24/10/2010, from http://www.rapppolymere.com/preise/scavres_d.htm.

33. Vickerstaffe, E.; Warrington, B. H.; Ladlow, M.; Ley, S. V. Org. Biomol. Chem. 2003, 1, 2419-2422.

34. Colby, D. A.; Lash, T. D. J. Org. Chem. 2002, 67, 1031-1033.

35. Lash, T. D.; El-Beck, J. A.; Colby, D. A. J. Org. Chem. 2009, 74, 8830-8833. 
36. Razus, A. C.; Birzan, L.; Nae, S.; Nitu, C.; Tecuceanu, V.; Cimpeanu, V. ARKIVOC 2002, 2, 142-153.

37. Franchi, E.; Ingrosso, G.; Marchetti, F.; Pinzino, C. Tetrahedron 2003, 59, 5003-5018.

38. McDonald, R. N.; Morris, D. L.; Petty, H. E.; Hoskins, T. L. Chem. Comm. 1971, 743-744.

39. Yan, B. Analytical methods in combinatorial chemistry; Technomic Publishing Company, 2000.

40. Grice, P.; Leach, A. G.; Massi, A.; Mynett, D. M.; Ley, S. V. J. Comb. Chem. 2000, 2, 491-495.

41. Treibs, W.; Vollrad, C.; Reimann, M. J. Liebigs. Ann. 1961, 648, 164-184.

42. Carpino, L. A.; Han, G. Y. J. Org. Chem. 1972, 37, 3404-3409.

43. Aumuller, I.; Lindhorst, T. K. J. Carbohydrate Chem. 2009, 28, 330-347.

44. Kaburagi, Y.; Osajima, H.; Shimada, K.; Tokuyama, H.; Fukuyama, T. Tet. Lett. 2004, 45, 3817-3821.

45. Soderquist, J. A.; Hassner, A. J. Organometal. Chem. 1978, 156, 227-233.

46. Cho, Y. S.; Yoo, B. R.; Ahn, S.; Jung, I. N. Bull. Korean Chem. Soc. 1999, 20, 427-430.

47. Hansch, C.; Leo, A.; W.Taft, R. Chem. Rev. 1991, 91, 165-195.

48. Treibs, W.; Schroth, W. J. Liebigs. Ann. 1954, 586, 202-212.

49. Voegtle, F.; Loehr, H.-G. Chem. Ber. 1985, 118, 905-913.

50. Zielinski, T.; Kedzioreka, M.; Jurczak, J. Tet. Lett. 2005, 6231-6234. 